\title{
Reversible amyloids of pyruvate kinase couple cell metabolism and stress granule disassembly
}

Gea Cereghetti

ETH Zurich

Caroline Wilson-Zbinden

ETH Zurich

Vera Kissling

ETH Zurich

Maren Diether

ETH Zurich

Alexandra Arm

ETH Zurich

Haneul Yoo

University of Chicago

llaria Piazza

ETH Zurich

Shady Saad

ETH Zurich

Paola Picotti

Swiss Federal Institute of Technology in Zurich

David Drummond

University of Chicago https://orcid.org/0000-0001-7018-7059

Uwe Sauer

ETH Zurich

\section{Reinhard Dechant}

Swiss Federal Institute of Technology in Zurich https://orcid.org/0000-0002-4094-4773

Matthias Peter ( $\triangle$ matthias.peter@bc.biol.ethz.ch )

ETH Zurich https://orcid.org/0000-0002-2160-6824

Article

Keywords: protein folding, protein aggregation, aging, enzymes

Posted Date: March 18th, 2021 
DOl: https://doi.org/10.21203/rs.3.rs-311638/v1

License: (c) (1) This work is licensed under a Creative Commons Attribution 4.0 International License. Read Full License

Version of Record: A version of this preprint was published at Nature Cell Biology on October 6th, 2021. See the published version at https://doi.org/10.1038/s41556-021-00760-4. 


\section{Reversible amyloids of pyruvate kinase couple cell} metabolism and stress granule disassembly

5 Gea Cereghetti ${ }^{1,2}$, Caroline Wilson-Zbinden ${ }^{1, *}$, Vera M. Kissling ${ }^{1,3, *}$, Maren Diether ${ }^{4}$, Alexandra

6 Arm $^{1}$, Haneul Yoo ${ }^{5}$, Ilaria Piazza ${ }^{4,7}$, Shady Saad ${ }^{1,6}$, Paola Picotti ${ }^{4}$, D. Allan Drummond ${ }^{5}$, Uwe Sauer ${ }^{4}$,

7 Reinhard Dechant ${ }^{1}$ and Matthias Peter ${ }^{1, \S}$

8

9

${ }^{1}$ Institute of Biochemistry, Department of Biology, ETH Zürich, Otto-Stern-Weg 3, 8093 Zürich, Switzerland

${ }^{2}$ Life Science Zürich, PhD Program for Molecular Life Sciences, 8057 Zürich, Switzerland

${ }^{3}$ Life Science Zürich, PhD Program for Biomolecular Structure and Mechanism, 8057 Zürich,

Switzerland

${ }^{4}$ Institute of Molecular Systems Biology, ETH Zürich, Otto-Stern-Weg 3, 8093 Zürich, Switzerland

${ }^{5}$ Department of Biochemistry and Molecular Biology, University of Chicago, Chicago, Illinois 60637, USA

${ }^{6}$ Current address: Department of Chemical and Systems Biology, Stanford University, 269 Campus Drive, CA 94305, USA

${ }^{7}$ Current address: Max-Delbrück Centre for Molecular Medicine, Robert-Rössle-Strasse 10, 13092 Berlin, Germany

* These authors contributed equally

$\S$ To whom correspondence should be addressed: 
Abstract

29

Cells respond to stress by blocking translation, rewiring metabolism, and forming transient mRNP assemblies called stress granules (SGs). After stress release, re-establishing homeostasis requires energy-consuming processes. However, the molecular mechanisms whereby cells restore energy production to disassemble SGs and reinitiate growth after stress remain poorly understood. Here we show that, upon stress, the ATP-producing enzyme Cdc19 forms inactive amyloids, and that their rapid re-solubilization is essential to restore energy production and disassemble SGs. Cdc19 re-solubilization is initiated by the glycolytic metabolite fructose-1,6-bisphosphate (FBP), which directly binds Cdc19 amyloids and facilitates conformational changes that allow Hsp104 and Ssa2 chaperone recruitment. FBP then promotes Cdc19 tetramerization, which boosts its activity to further enhance ATP production and SG disassembly. Together, these results describe a molecular mechanism essential for stress recovery, which directly couples metabolism with SG dynamics via regulation of Cdc19 amyloids. 


\section{Introduction}

Cells respond to diverse intra- and extracellular signals as well as environmental changes, and adjust their activities to maintain homeostasis. In particular, cells need to rapidly respond to potentially harmful stress conditions such as osmolarity changes, oxidative or heat stress and nutrient starvation [1]. To cope with these challenges, cells developed complex mechanisms to sense various stress conditions and orchestrate appropriate protective responses, including inhibition of cell growth and cell cycle progression, activation of specific transcriptional programs, and formation of mRNP assemblies known as stress granules (SGs) $[1,2]$. SGs are dynamic cytoplasmic membrane-less organelles that transiently form in response to metabolic stress and are rapidly disassembled under favourable growth conditions. They contain several RNA-binding proteins and metabolic enzymes [3], and are thought to play an important role in maintaining homeostasis by sequestering cellular components and protecting them from stress-induced degradation, and/or by concentrating them to favour specific interactions [46]. Alterations in SG dynamics and the consequent metabolic changes have been implicated in the pathogenesis of several human neurodegenerative diseases such as amyotrophic lateral sclerosis (ALS), Alzheimer's disease (AD) and frontotemporal lateral dementia (FTD) [7, 8]. Indeed, chronic stress or mutations leading to persistent SGs have been shown to facilitate the formation of irreversible, pathological protein aggregates, suggested to promote aging and aging-associated diseases [8]. SGs thus need to be carefully controlled and efficiently disassembled. However, despite their pathophysiological importance, the mechanisms regulating SG dynamics remain poorly understood.

Most SG proteins contain intrinsically disordered low-complexity regions (LCRs) that are able to condense by liquid-liquid phase separation (LLPS) $[9,10]$. Together with low-affinity RNA-RNA interactions, this LLPS process is thought to underlie, at least in part, the formation of SGs [11, 12]. Several factors are known to influence LLPS, including post-translational modifications, energy availability, temperature, and biophysical properties of the cytoplasm, such as crowding and viscosity [13-17]. In contrast, the mechanisms regulating SG disassembly are less well understood, but ATP seems to play a crucial role in this process. Indeed, artificial ATP depletion reduces SG dynamics and results in persistent SGs $[3,9,18]$. In contrast, adding ATP to stressed cells is sufficient to trigger 
aggregate disassembly [19]. Thus, ATP availability is essential and sufficient to initiate SG disassembly, and energy-consuming activities including autophagy, as well as ATP-dependent enzymes such as chaperones and RNA/DNA helicases have been implicated in this process [20-26]. For instance in yeast, the disaggregase Hsp104 and the Hsp70 chaperones Ssa1 and Ssa2 accumulate in SGs, and their ATP-dependent activities are important for cells to efficiently disassemble SGs and restart growth after stress release [19, 20]. The ATP-dependent kinases Sky1 [22] and ULK1/2 [23] have also been implied in efficient SG disassembly, and ATP itself has been proposed to act as a biological hydrotrope to modulate cytoplasmic diffusion and further facilitate aggregate re-solubilization [27]. Intriguingly, however, ATP reserves are rapidly depleted in stressed cells [19, 28-31], raising the question of how energy production is restored after stress release to allow efficient SG disassembly.

Cells mainly produce ATP by fermenting glucose through glycolysis $[32,33]$. In this pathway, the ATP-producing and irreversible final step is carried out by the essential and evolutionarily conserved enzyme pyruvate kinase (Cdc19 in yeast), which catalyses the conversion of phosphoenolpyruvate (PEP) and ADP into pyruvate and ATP. Cdc19 activity is influenced by its allosteric regulator, fructose-1,6-biphosphate (FBP), whose binding promotes Cdc19 oligomerization into more active tetramers $[4,31,34,35]$. Moreover, flux through glycolysis is modulated by trehalose metabolism, which regulates glucose-6-phosphate availability and hexokinase activity [36-38]. Interestingly, we previously showed that in response to glucose starvation and heat stress, Cdc19 reversibly aggregates via its aggregation-prone LCR and localizes to SGs [4, 39]. Formation and disassembly of Cdc19 aggregates modulate SG dynamics, and play a crucial role in stress survival. Indeed, aggregating Cdc19 protects this essential enzyme from stress-induced degradation, and accelerated Cdc19 aggregation leads to faster SG formation. In contrast, re-solubilization of Cdc19 aggregates is required to dissolve SGs and re-activate TORC1 and PKA growth pathways after stress release [4]. Mutations in the Cdc19 LCR that cause irreversible aggregates are also shown to compromise SG disassembly and cell cycle restart after stress. Cdc19 is remarkable in that it forms stable amyloid-like aggregates in vitro, while in vivo these aggregates are fully reversible within minutes after stress release. As amyloids are characterized by high resistance to enzymatic degradation, 
this conformation would be well suited to avoid degradation during stress in vivo, but would raise the question of how these structures can be readily disassembled once stress is released. However, the structural nature of physiological Cdc19 aggregates, and the molecular mechanisms governing their disassembly in vivo remain unclear. Likewise, the physiological relevance of reversible amyloid-like Cdc19 aggregates in SG regulation and growth restart after stress is poorly understood.

To address these questions, we exploited a mutant of $\mathrm{Cdc} 19\left(\mathrm{Cdc} 19^{\text {irrev }}\right)$ which is unable to disassemble Cdc19 aggregates after stress [4], and combined in vitro and in vivo approaches to elucidate the molecular mechanisms responsible for Cdc19 re-solubilization. Moreover, we further characterized the structure of reversible Cdc19 aggregates in vivo, showing that they assume an amyloid structure also within cells. Interestingly, we found that formation of Cdc19 amyloids inactivates the enzyme, thus abolishing ATP production during stress. Re-solubilization of these aggregates after stress release is necessary to restore ATP production and re-solubilize SGs, and we propose a molecular mechanism describing how functional Cdc19 amyloids are disassembled. In particular, we demonstrate that direct binding of FBP to Cdc19 amyloids initiates disassembly by promoting a conformational change that is required to trigger recruitment of the molecular chaperones Hsp104 and Ssa2, and to allow Cdc19 amyloid re-solubilization and subsequent SG disassembly. Together, our studies not only provide a mechanism for re-solubilizing functional amyloids in vivo, but also show how metabolism and energy production are coupled to SG dynamics and cell growth by regulating both assembly and disassembly of reversible, functional Cdc19 amyloids.

\section{Results}

\section{Re-solubilization of Cdc19 amyloids is required to restore ATP production and disassemble SGs}

Formation of reversible Cdc19 aggregates upon stress is required to ensure cell survival [4]. We found that upon return to favourable growth conditions, re-solubilization of Cdc19 aggregates is essential to both disassemble SGs and re-initiate growth [4]. Indeed, cells expressing a functional but irreversibly aggregating mutant of $\mathrm{Cdc} 19$ (Cdc19 $9^{\text {irrev }}$, Suppl. Fig 1A) are unable to survive stress and 
cannot disassemble SGs (Fig. 1A). Interestingly, Cdc19 $9^{\text {irrev }}$ aggregates not only fail to re-solubilize after stress, but also cause persistent SGs (Fig. 1A). In order to understand how Cdc19 regulates SG disassembly and the mechanisms underlying Cdc19 aggregate re-solubilization, we investigated wildtype and Cdc1 $19^{\text {irrev }}$ aggregate properties and potential differences in vitro and in vivo.

First, we examined biophysical properties of wild-type and Cdc19 $9^{\text {irrev }}$ aggregates, to understand if irreversibility was caused by an aberrant aggregate structure. To this end, wild-type and mutant proteins were expressed and purified from E. coli, and their aggregation was triggered in vitro with a brief heat shock treatment. Upon heat shock, both proteins formed needle-like filaments, which were indistinguishable by negative staining transmission electron microscopy (TEM) analysis (Fig. 1B). Both wild-type and mutant aggregates also revealed similar $\beta$-sheet rich-structures, as shown by circular dichroism (CD) measurements (Fig. 1C), and could bind the amyloid dyes Thioflavin T (ThT) and Congo Red (CR) [40] (Suppl. Fig. 1B and C), suggesting that wild-type and Cdc19 $9^{\text {irrev }}$ proteins form similar amyloids in vitro. To test whether Cdc19 aggregates exhibit amyloid structures in vivo, we subjected yeast cells to heat shock, lysed them, and stained both wild-type and Cdc19 $9^{\text {irrev }}$ aggregates with CR. As shown in Suppl. Figure 1D, cellular Cdc19 aggregates could be stained with the amyloid dye in both cases, while no Cdc19 aggregates were visible in control conditions. Background CR staining is likely due to the ability of CR to bind cellular structures (e.g. cell wall) other than amyloids [41]. To further confirm the amyloid nature of Cdc19 aggregates in vivo, and to better compare the structure of wild-type and Cdc19 $9^{\text {irrev }}$ aggregates, we applied limited proteolysis coupled to massspectrometry (LiP-MS). This method allows to probe subtle conformational changes both in vitro and in complex cell lysates, and offers more precise structural information compared to conventional methods for detecting amyloids $[42,43]$. Thus, we used LiP-MS to compare the structural alterations undergone by wild-type and $\mathrm{Cdc} 19^{\text {irrev }}$ upon aggregation in vivo and in vitro (Suppl. Fig. 1E). Cdc19 aggregation was induced in vitro by heat shocking purified Cdc19, and in vivo by subjecting cells to starvation stress to achieve full amyloid formation. This analysis revealed that diagnostic Cdc19 LiPMS peptides characteristic of the amyloid conformation in vitro resemble the structures observed in vivo, thus corroborating the notion that $\mathrm{Cdc} 19$ aggregates are amyloids both in vitro and in vivo. 
Moreover, conformation-sensitive LiP-peptides were mostly similar in wild-type and Cdc19 $9^{\text {irrev }}$ assemblies, suggesting that the different re-solubilization behaviour of these proteins is not due to the formation of two radically different structures (Suppl. Fig. 1E). However, LiP-MS identified subtle structural alterations in the aggregated catalytic domain of Cdc19. We therefore asked whether Cdc19 activity is affected by its aggregation and re-solubilization, and examined potential differences between $\mathrm{Cdc} 19^{\mathrm{WT}}$ and $\mathrm{Cdc} 19^{\text {irrev }}$.

First, we compared the enzymatic activity of soluble and aggregated Cdc19 using an established lactate-dehydrogenase-coupled pyruvate kinase activity assay. Cdc19 amyloids were enzymatically inactive (Fig. 1D), suggesting that the active site in these reversible assemblies may be inaccessible to convert PEP into ATP. Given the large conformational changes undergone by the protein to form amyloid fibrils, the loss of catalytic activity is not surprising. This is also in accordance with previous observations showing that in cells exposed to heat stress or glucose starvation (i.e. when Cdc19 is aggregated) ATP levels rapidly drop, while conversely Cdc19's substrate PEP accumulates under these conditions [19, 29-31] (Fig. 1F). To re-activate Cdc19 and resume ATP production, Cdc19 amyloids thus need to be re-solubilized. Since high ATP levels are essential to disassemble SGs, we asked whether persistent SGs in $c d c 19^{\text {irrev }}$ cells could be due to impaired ATP production. Indeed, we found that while wild-type cells presented elevated ATP levels and low PEP during stress recovery, $c d c 19^{\text {irrev }}$ cells could not metabolize the accumulated PEP and hence failed to restore ATP production (Fig. 1E). We conclude that wild-type and $c d c 19^{\text {irrev }}$ cells differ primarily in their ability to restore energy production after stress.

To test if increasing ATP levels was sufficient to disassemble SGs, we restored energy metabolism in heat stressed $c d c 19^{\text {irrev }}$ cells by two different Cdc19-independent means. First, we artificially overexpressed Pyk2, a pyruvate-kinase isoform that is expressed at very low levels in glucose-rich conditions but like Cdc19 uses PEP to produce ATP [44]. In accordance with our hypothesis, restoring ATP production by overexpressing Pyk2 re-solubilized Cdc19 $9^{\text {irrev }}$ aggregates and SGs, and restored growth of $c d c 19^{\text {irrev }}$ cells exposed to heat stress (Fig. $1 \mathrm{G}$ and H). Second, we grew $c d c 19^{\text {irrev }}$ cells with $2 \%$ ethanol instead of glucose. Under these conditions, ATP production occurs 
mainly via oxidative phosphorylation independently of Cdc19 [45]. Also in this case, having an alternative ATP source allowed re-solubilization of $\mathrm{Cdc} 19^{\text {irrev }}$ aggregates and SGs (Fig. 1I). We conclude that under physiological conditions, re-solubilizing Cdc19 amyloids is critical to resume ATP production after stress, which in turn is likely required to power ATP-dependent processes to dissolve SGs and restart cell growth.

\section{Re-solubilization of Cdc19 amyloids in vivo is triggered by its allosteric regulator fructose-1,6- bisphosphate (FBP)}

To better understand the molecular mechanisms regulating Cdc19 amyloid disassembly, we performed a genetic suppressor screen to identify mutants that are able to restore growth of stressed $c d c 19^{\text {irrev }}$ cells (Suppl. Fig. 2A). Briefly, we subjected exponentially growing cells expressing GFPtagged $\mathrm{Cdc} 19^{\text {irrev }}$ to a $42{ }^{\circ} \mathrm{C}$ heat shock treatment for $30 \mathrm{~min}$, and plated the cells at $30{ }^{\circ} \mathrm{C}$ to isolate rare spontaneous suppressors which were able to resume growth after stress. We deleted pyk 2 in the isolated mutants to exclude the possibility that suppression is mediated by upregulating Pyk2 activity, and then backcrossed to wild-type cells to confirm Mendelian segregation of the phenotype. Using whole genome sequencing, we identified a single point mutation in Tps3, a protein involved in trehalose biosynthesis, as the likely cause for the Cdc19 $9^{\text {irrev }}$ suppressor phenotype (Suppl. Fig. 2B and C). Trehalose is a disaccharide which is synthesized in a two-steps process (Fig. 2A). In a first step, Tps1 catalyses the conversion of glucose-6-phosphate (G6P) into trehalose-6-phosphate (T6P), and Tps2 then converts T6P into trehalose. Tps3 acts as cofactor, facilitating this trehalose production. Deletion of either tps1, tps2, or tps3, which abrogates trehalose production (Suppl. Fig. 2E, [46]), did not alter growth compared to wild-type controls (Suppl. Fig. 2D), but allowed $c d c 19^{\text {irrev }}$ cells to overcome stressinduced growth arrest and partially restored growth after stress release (Fig. 2B), suggesting that decreasing trehalose metabolism favours Cdc19 re-solubilization. As Tps1 catalyses the first reaction in the trehalose synthesis pathway and its deletion resulted in the strongest rescue (Fig. 2B), we first focussed on this mutant. Fluorescence microscopy confirmed that growth restart correlated with resolubilization of Cdc19 irrev amyloids and SG disassembly in the tps 14 strain (Fig. 2C). As expected, Cdc19 re-solubilization in turn coincided with restored ATP production after stress release. Indeed, 
metabolomic data showed that $c d c 19^{\text {irrev }}$ cells fail to produce high ATP concentrations and instead maintain high intracellular PEP levels after stress release, while $c d c 19^{\text {irrev }}$ tps $1 \Delta$ cells consume PEP and can resume ATP production (Fig. 2D).

We next wanted to understand how Tps1 deletion allows $c d c 19^{\text {irrev }}$ amyloid disassembly. We reasoned that Tps1 activity decreases glycolytic flux on the one hand by diverting G6P from glycolysis into trehalose synthesis, and on the other hand by generating T6P, which in turn inhibits hexokinase (Fig. 2A, [36-38]). As a result, tps $1 \Delta$ cells contain virtually no trehalose, but have enhanced flux through glycolysis, which results in increased levels of the glycolytic intermediate fructose-1,6-bisphosphate (FBP) [38]. FBP levels tightly correlate with glycolytic flux and cell growth, with low FBP reflecting low glycolysis and a stressed, quiescent cell state, while high FBP levels denote high glycolytic flux and metabolic conditions favourable for growth $[47,48]$. Interestingly, besides being a "glycolysis flux sensor", FBP is also a known allosteric regulator of Cdc19 [31]. We thus hypothesized that increased FBP levels in tps $1 \Delta$ cells may directly or indirectly play a role in the disassembly of Cdc19 aggregates. To test this, we compared intracellular trehalose and FBP levels in $c d c 19^{\text {irrev }}$ and $c d c 19^{\text {irrev }}$ tps $1 \Delta$ cells exposed to heat shock conditions. As expected, deletion of Tps1 abrogated the production of the disaccharide trehalose (Fig. 2E). Moreover, similar to glucose starvation [38], heat stress induced a rapid decrease in FBP, but during stress recovery this metabolite then accumulated to significantly higher levels in $c d c 19^{\text {irrev }} \operatorname{tps} 1 \Delta$ mutants compared to $c d c 19^{\text {irrev }}$ controls (Fig. 2F). To genetically test whether the observed rescue of stressed Cdc1 $9^{\text {irrev }}$ mutants by tps $1 \Delta$ is mediated by increased FBP levels, we deleted Pfk2, the main FBP-producing enzyme in glycolysis, to impair FBP production [38]. Interestingly, in contrast to tps $1 \Delta$, tps $1 \Delta p f k 2 \Delta$ double mutants were unable to recover from heat stress, while $p f k 2 \Delta$ alone neither significantly reduced growth rates nor impaired survival to stress (Fig. 2G), despite comparable expression levels of the Cdc19 variants (Suppl. Fig 2F). Moreover, tps1 $\Delta$ pfk $2 \Delta$ cells were unable to dissolve Cdc19 $9^{\text {irrev }}$ aggregates and SGs (Fig. $2 \mathrm{H}$ ). Thus, we conclude that high FBP levels contribute to efficient stress recovery and Cdc19 amyloid and SG disassembly. 

trehalose has been shown to affect SG dynamics by tuning cytoplasmic viscosity [15]. Indeed, also deleting Tps2, the enzyme catalysing the last step in the trehalose synthesis pathway, weakly rescues the stress recovery and aggregate dissolution defects observed in $c d c 19^{\text {irrev }}$ cells (Suppl. Fig. 2G), although this effect is much weaker compared to tps $1 \Delta$ cells (Fig. 2B). While both strains are impaired in trehalose synthesis, only deletion of Tps1, and not Tps2, resulted in increased FBP levels after stress in $c d c 19^{\text {irrev }}$ cells (Suppl. Fig. 2H). We thus conclude that FBP-dependent and -independent mechanisms might co-operate to achieve efficient Cdc19 amyloid disassembly upon stress release, although increasing cytoplasmic viscosity via trehalose breakdown plays a minor role compared to increased FBP levels.

\section{FBP directly regulates the formation and disassembly of reversible Cdc19 amyloids}

Our results suggest that FBP serves as a sensor to couple cellular metabolism and energy production with SG disassembly and cell growth, by directly modulating Cdc19 aggregation and catalytic activity (Fig. 2). Two mechanisms could be envisioned to explain how direct FBP binding could regulate this process (Fig. 3A). First, FBP could reduce aggregation by promoting Cdc19 tetramerization (Fig. 3A, arrow 1), as Cdc19 tetramers are less aggregation-prone than monomers [4]. Alternatively, FBP could directly bind to Cdc19 amyloids and induce conformational changes that lead to Cdc19 amyloid and SG disassembly (Fig. 3A, arrow 2). These two modes of action are not mutually exclusive, and could co-operate to ensure rapid Cdc19 re-solubilization. To elucidate the mechanism underlying FBP-mediated regulation of Cdc19 amyloids, we next investigated the effects of FBP on the formation and disassembly of Cdc19 aggregates. First, we biochemically compared Cdc19 amyloid formation in the presence or absence of FBP and other metabolites in vitro (Fig. 3B-D). Incubation of purified Cdc19 ${ }^{\mathrm{WT}}$ with $5 \mathrm{mM}$ FBP, but not its structurally-related precursor fructose-6-phosphate (F6P), interfered with aggregate formation as demonstrated by a simple centrifugation assay (Fig. 3B). Indeed, in the presence of FBP, a significant portion of $\mathrm{Cdc} 19^{\mathrm{WT}}$ remained soluble, with a corresponding decrease in the aggregate pellet fraction. Conversely, addition of $5 \mathrm{mM} \mathrm{F6P,} \mathrm{ATP,} \mathrm{PEP} \mathrm{or} \mathrm{trehalose} \mathrm{did}$ not influence Cdc19 aggregation (Suppl. Fig. 3A-C). To confirm that FBP reduces Cdc19 aggregation 
by directly binding to the protein, we next measured the aggregation behaviour of a Cdc19 point-mutant (T403E) unable to bind FBP (Cdc19 ${ }^{\triangle \mathrm{FBP}}$, [49]). As shown in Figure 3C, addition of FBP did not interfere with heat shock-induced pelleting of $\mathrm{Cdc} 19^{\triangle \mathrm{FBP}}$. Taken together, these data show that direct binding of FBP to Cdc19 monomers prevents amyloid formation in part by promoting the formation of nonaggregating Cdc19 tetramers (Fig. 3A, arrow 1). However, FBP did not affect the formation of Cdc19 irrev aggregates (Fig. 3D), suggesting that this mutant might have a reduced FBP-binding ability and/or be unable to tetramerize. Indeed, size-exclusion chromatography demonstrated that the $\mathrm{Cdc} 19^{\text {irrev }}$ protein is fully monomeric both in vivo and in vitro (Fig. 3E and F). Importantly, these results imply that the FBP-mediated rescue of the Cdc19 $9^{\text {irrev }}$ mutant in vivo cannot simply be explained by increased tetramerization (Fig. 3A, arrow 1), but rather FBP must also promote aggregate disassembly (Fig. 3A, arrow 2). To confirm that direct binding of FBP to Cdc19 amyloids is required for their disassembly, we compared aggregation dynamics of wild-type Cdc19 and the FBP binding-deficient $\mathrm{Cdc} 19^{\triangle \mathrm{FBP}}$ mutant in vivo (Fig. 3G). Interestingly, while the $\mathrm{Cdc} 19^{\triangle \mathrm{FBP}}$ mutant protein was soluble under favourable growth conditions and rapidly aggregated upon heat shock, Cdc19 $9^{\triangle \mathrm{FBP}}$ aggregates were irreversible and unable to disassemble after stress release. In contrast to Cdc19irrev aggregates, Cdc19 $9^{\triangle \mathrm{FBP}}$ aggregates could not be rescued by increasing FBP levels by TPS1 deletion. Taken together, these data show that direct binding of FBP to Cdc19 is essential to disassemble Cdc19 amyloids in vivo.

Next, we examined whether FBP is sufficient to disassemble pre-formed Cdc19 amyloids in vitro. We induced fibril formation of purified wild-type Cdc19 by heat shock, and incubated the obtained amyloids with varying concentrations of FBP. After several hours, the aggregates were pelleted by centrifugation and the soluble and insoluble fractions analysed by SDS-PAGE. As shown in Suppl. Fig. 3 D, we did not observe significant re-solubilisation of Cdc19 even upon incubation with excess FBP (20 mM), indicating that FBP binding is not sufficient to disassemble Cdc19 amyloids in vitro. However, we found that FBP is able to alter the morphology of Cdc19 amyloids. Indeed, incubation of pre-formed $\mathrm{Cdc} 19^{\mathrm{WT}}$ aggregates with FBP resulted in clear morphological alterations as shown by negative staining TEM (Fig. 3H, +FBP). Formerly straight fibrils developed protruding globular bulges and several regions of mild curvature along the fibril axis. Furthermore, larger globular 
assemblies appeared close to the fibrils, and the fibrils were consistently positively stained (i.e. of dark colour). This change from negative (white fibrils) to positive (black fibrils) staining results from acidic FBP molecules bound to the fibrils that attract significantly more of the positively charged uranyl stain. In contrast, incubation of pre-formed Cdc19 ${ }^{\mathrm{WT}}$ amyloids with buffer (Fig. 3H, buffer) or the FBP "analogue" F6P (Fig. 3H, +F6P) did not cause any morphological changes and the Cdc19 ${ }^{\mathrm{WT}}$ amyloids appeared as needle-like fibrils characterized by smooth edges, thus confirming FBP-specificity. As expected, Cdc19 $9^{\triangle \mathrm{FBP}}$ amyloid fibrils incubated with FBP also retained their straight, smooth morphology, confirming that the observed conformational changes are induced by direct FBP binding to $\mathrm{Cdc} 19$ (Fig. 3H, lower panels). Together, these striking differences suggest that FBP directly binds to Cdc19 amyloids and induces pronounced conformational changes along the fibril. While these alterations are not sufficient to trigger Cdc19 amyloid re-solubilization in vitro, they may facilitate binding of other factors that together promote the efficient amyloid disassembly observed in vivo.

\section{Chaperones co-operate with FBP to achieve efficient re-solubilization of Cdc19 amyloids in vivo}

Untangling of cellular misfolded protein aggregates is mediated by different chaperones, including Hsp104 and members of the Hsp70 (Ssa1-4) and the Hsp110 families (Sse1-2) [20, 50, 51]. Certain chaperones, such as Hsp104 and Ssa2, have also been implicated in re-modelling functional protein assemblies and SGs, and indeed cells lacking HSP104 or SSAl/2 are slightly delayed in SG disassembly [50]. We thus asked whether these chaperones could also be involved in facilitating disaggregation of Cdc19 amyloids. Interestingly, we observed that inhibiting Hsp104 activity using $\mathrm{Gdn}-\mathrm{HCl}$ caused a delay in dissolution of both wild-type Cdc19 aggregates, as well as Cdc19 $9^{\text {irrev }}$ aggregates in tps $1 \Delta$ cells (Fig. 4A and B). Conversely, overexpressing Hsp104 was sufficient to at least partially restore growth of heat stressed $c d c 19^{\text {irrev }}$ cells (Fig. 4C), and this effect was further enhanced by simultaneously co-overexpressing Ssa2 (Suppl. Fig. 4A and B). Moreover, Hsp104 overexpression slightly improved growth of $c d c 19^{\text {irrev }}$ tps $1 \Delta$ mutants after heat stress, indicating that increased FBP and chaperone levels have an additive effect (Fig. 4D). Increasing FBP levels by TPS1 deletion allowed cells to rapidly restart growth after stress release, while Hsp104 overexpression seemed to take effect in facilitating growth at a later phase, suggesting that high FBP may be particularly important in the 
initial steps of Cdc19 amyloid disassembly (Suppl. Fig. 4C). Together, these results imply that FBP binding to Cdc19 initiates aggregate re-solubilization and co-operates with Hsp104 and Ssa2 to disassemble SG in vivo. To further investigate the interplay between FBP levels and chaperones in the disassembly of Cdc19 amyloids, we compared the localization of Cdc19-GFP and Hsp104-mCherry or Ssa2-mCherry in wild-type, $c d c 19^{\text {irrev }}$ and $c d c 19^{\text {irrev }}$ tps $1 \Delta$ cells. As expected, fluorescence microscopy confirmed that Hsp104-mCherry and Ssa2-mCherry co-localized with heat shock-induced Cdc19-GFP aggregates in the majority of wild-type cells (Fig. 4E and F). Surprisingly, however, Hsp104 and Ssa2 were not recruited to irreversible $\mathrm{Cdc} 19^{\text {irrev }}$ aggregates but rather remained dispersed throughout the cytoplasm. Co-localization was restored upon deletion of tps 1 , consistent with the notion that increased FBP levels and FBP binding to Cdc19 allow chaperone recruitment to SGs (Fig. 4E and F) to promote Cdc19 and SG re-solubilization (Suppl. Fig. 4D). To corroborate these results, we next examined colocalization of Hsp104-mCherry with GFP-tagged Cdc19 $9^{\triangle \mathrm{FBP}}$. Indeed, Cdc19 $9^{\triangle \mathrm{FBP}}$ amyloids failed to recruit Hsp104-mCherry, thus confirming that FBP binding to Cdc19 is essential for chaperone recruitment (Fig. 4G). In this background Hsp104-mCherry showed a punctate pattern by binding to aggregates formed upon heat stress, but these foci did not co-localize with irreversible Cdc19 $9^{\triangle \mathrm{FBP}}$ amyloids (Fig. 4G). As expected, co-localization with $\mathrm{Cdc} 19^{\triangle \mathrm{FBP}}$ aggregates was not restored in tps $1 \Delta$ cells. We thus conclude that FBP-binding to Cdc19 aggregates triggers a conformational change that promotes chaperone binding and allows their recruitment to SGs, which in turn is essential for Cdc19 amyloid re-solubilization, resumption of ATP production, and SG disassembly.

\section{Discussion}

Cell survival under stress conditions requires rapid and reversible implementation of protective measures, including reprogramming of cellular metabolism, cytoplasmic re-organization and activation of selective autophagy [1]. A key step in this process is the formation of stress granules (SGs), highly dynamic, cytoplasmic, membrane-less organelles that are thought to store and protect mRNAs as well as proteins from stress-induced degradation [3]. Formation and disassembly of SGs needs to be tightly 
regulated, as persistent SGs have been associated with several human pathologies [3]. Among the proteins regulating SG dynamics is the ATP-producing enzyme Cdc19. Cdc19 assembles into solid but reversible aggregates and localizes to SGs upon stress [4]. Once cells are released from stress, resolubilization of Cdc19 aggregates is an essential step in disassembling SGs and restoring cell growth. However, the mechanisms regulating Cdc19 aggregate re-solubilization and their role in SG disassembly was poorly understood. Here, we show that reactivating energy metabolism is essential for SG disassembly, and propose a mechanism that links cellular metabolism and energy production to the assembly state of Cdc19.

\section{Cdc19 forms catalytically inactive, reversible amyloids in response to stress conditions}

Untimely degradation of essential proteins such as Cdc19 has lethal consequences. During stress, proteosomal degradation is upregulated [52] and misfolded proteins are often degraded through various pathways [53]. Therefore, essential proteins required for restarting growth need to be protected from degradation [4]. Several lines of evidence suggest that stress-induced Cdc19 aggregates assemble into a proteasome-resistant amyloid structure, similar to irreversible pathological aggregates characteristic of neurodegenerative diseases [39], while preserving the capacity to resolubilize after returning to stress-free conditions. Indeed, our CD measurements, amyloid-specific dye stainings, and TEM analyses demonstrate that purified Cdc19 forms amyloids in vitro. Furthermore, in vivo FRAP measurements [4] and staining of Cdc19 aggregates isolated from permeabilized yeast cells with the amyloid-specific dye Congo Red indicate that Cdc19 aggregates acquire solid, amyloid structures also in vivo. Finally, structural analysis by LiP-MS confirmed that Cdc19 aggregates in vivo resemble the amyloids formed by purified proteins in vitro. Taken together, we conclude that Cdc19 aggregates avoid stress-induced degradation in vivo owing to their proteolysis-resistant amyloid structure. These rigid assemblies may also have a scaffolding function and influence the structural integrity and dynamics of SGs. The comparison of $\mathrm{Cdc} 19^{\mathrm{WT}}$ and $\mathrm{Cdc} 19^{\text {irrev }}$ aggregates further highlights that the structural features of physiological, reversible amyloids and irreversible structures are remarkably similar. It is worth mentioning that even single amino acid changes can be sufficient to transform reversible aggregates into aberrant entities associated with diseases, even without major structural changes [54]. 

its activity and hence ATP production. The formation of catalytically inactive Cdc19 amyloids results in a rapid decrease of cellular ATP levels and accumulation of glycolytic intermediates such as PEP during stress [31]. Thus, stress-induced Cdc19 aggregates are distinct from glycolytic bodies, which are thought to increase glycolytic flux in response to hypoxic stress by coalescing glycolytic enzymes [55]. Therefore, while in some cases concentrating molecular interactions or fixing favourable conformations promotes enzymatic reactions, the stress-induced structural rearrangements of Cdc19 prevent catalytic activity. While LiP-MS measurements exposed small conformational changes in the catalytic site, detailed structural information is required to understand how catalysis is inhibited in Cdc19 amyloids. Nevertheless, it is likely that the catalytic site undergoes a conformational change and/or becomes inaccessible to substrate when Cdc19 monomers assemble into higher order structures upon stress. In summary, forming solid amyloids protects Cdc19 from degradation and inhibits glycolysis during stress, while preserving the rapid re-initiation capability of cell growth.

FBP binding regulates disassembly of Cdc19 amyloids by recruiting molecular chaperones

Our data show that signals from glycolysis and cell cycle converge onto Cdc19, which in turn controls SG dynamics and cell survival during stress. Previous experiments indicate that FBP levels “metabolic sensor" for glycolytic flux.

Our results suggest that FBP regulates the assembly state and activity of Cdc19 by two mechanisms (Fig. 5). First, during exponential growth, FBP binds to soluble Cdc19, promoting its tetramerization. In tetramers, the aggregation-prone LCR of Cdc19 forms an $\alpha$-helix buried at the subunit interface, and is thus inaccessible to seed amyloids [4]. In contrast, low FBP levels characteristic of stressed conditions allow the formation of Cdc19 monomers, and the exposed LCR can trigger aggregation. Second, upon stress release, glycolysis restarts and FBP levels reach a critical concentration that facilitates Cdc19 re-solubilization by directly binding Cdc19 amyloids, inducing 
conformational changes needed to trigger their disassembly. Indeed, cells expressing Cdc19 ${ }^{\triangle \mathrm{FBP}}$ mutant protein, which cannot bind FBP, form aggregates that are unable to disassemble after stress release. Genetically increasing FBP levels by deleting TPS1 could not rescue this phenotype, but was sufficient to allow disassembly of amyloids formed by the otherwise irreversible Cdc19 irrev mutant. Since the Cdc19 $9^{\text {irrev }}$ protein is exclusively monomeric in vitro and in vivo, it is likely that it exhibits decreased FBP binding affinity, which can be overcome by increased FBP levels. Although FBP is not sufficient to disassemble amyloid Cdc19 fibrils in vitro, TEM analysis showed that FBP binding directly triggers profound morphological alterations along the entire fibrils axis that may help to dissolve these rigid structures. Based on these data we conclude that FBP links cellular metabolism to Cdc19 activity and regulates both the formation and disassembly of Cdc19 amyloids in vivo.

The dissolution of aggregated proteins commonly requires the help of molecular chaperones. In yeast, Hsp104 and Ssa1/Ssa2 play important roles in both counteracting and clearing aberrant protein aggregates [20,51], as well as in regulating the formation and disassembly of functional structures such as SGs. Interestingly, we found that following FBP binding to Cdc19 aggregates, Hsp104 and Ssa2 are required for re-solubilization. Both Hsp104 and Ssa2 co-localize with Cdc19 amyloids in SGs, and their ATP-dependent activity contributes to efficiently dissolve these reversible structures after stress release. In contrast, $\mathrm{Cdc} 19^{\triangle \mathrm{FBP}}$ and $\mathrm{Cdc} 19^{\text {irrev }}$ amyloids fail to recruit Hsp104 and Ssa2, and consequently cannot disassemble after stress release. In $c d c 19^{\text {irrev }}$ cells, Hsp104 and Ssa2 remain dispersed throughout the cytoplasm. Since substrate binding of Hsp104 is ATP-dependent [56], this effect is likely due to the low ATP levels after stress in these cells. As expected, co-localization with Cdc19 amyloids is restored in $c d c 19^{\text {irrev }}$ tps $1 \Delta$ cells, where ATP production can be resumed. Surprisingly, however, Hsp104 and Ssa2 fail to co-localize with irreversible amyloids formed by $\mathrm{Cdc} 19^{\triangle \mathrm{FBP}}$, even when ATP-production is restored by overexpressing Pyk2. In these mutant cells, both Hsp104 and Ssa2 form cytoplasmic puncta upon stress, as they are also needed to dissolve other aggregates formed by misfolded proteins, but do not co-localize with Cdc19. Chaperones recognize misfolded proteins by the exposure of hidden hydrophobic domains or specific sequences. Our results suggest that the conformational change triggered by FBP binding to Cdc19 amyloids not only initiates release of individual Cdc19 monomers, 
but also forms a binding site for efficient chaperone recruitment. Indeed, an algorithm predicting potential Hsp70-binding sites [57] identifies a likely chaperone binding site in Cdc19, which corresponds to the FBP-binding site. We conclude that Cdc19 amyloids are physiological targets of Hsp104 and Ssa2, but FBP-binding is required to help chaperone recruitment and enhance Cdc19 amyloid re-solubilization in vivo. Future work will be required to explore mechanistic differences in recognition and activity of chaperones towards physiological and pathological targets.

\section{Re-solubilization of Cdc19 amyloids restores ATP production to allow SG disassembly} and SGs (Fig. 5). Stressed cells suffer from low ATP levels, and ATP synthesis is a pre-requisite for SG disassembly and restart of growth-promoting processes [19]. Thus, upon stress-release, increasing FBP levels and binding to Cdc19 amyloids triggers a conformational change, which initiates disassembly and increases catalytic activity of $\mathrm{Cdc} 19$ to start converting the readily available PEP into ATP. FBP binding together with increasing ATP allows recruitment of the chaperones Hsp104 and Ssa2, which further disentangle Cdc19 fibrils, resulting in even more ATP production. This positive feedback loop rapidly solubilizes Cdc19 monomers, which in the presence of increasing FBP concentrations assemble into fully active tetramers [4]. The need for ATP production by Cdc19 can be bypassed with non-glycolytic substrates, which are metabolized by oxidative phosphorylation in mitochondria. While Hsp104 was identified as the critical ATP-consuming enzyme during this process [19], other cellular factors such as RNA/DNA-helicases, protein kinases and AAA-ATPases may also contribute to further dissolve SGs and restart cell growth [9]. In addition, metabolic changes can alter the physico-chemical properties of the cytoplasm, such as viscosity or crowding, which in turn may influence aggregation. For instance, recent experiments revealed that stress-induced changes in trehalose and glycogen levels tune cytoplasmic diffusion, thereby altering phase separation [15]. While our results suggest that regulated trehalose levels contribute to Cdc19 disassembly in vivo, this effect is rather minor compared to FBP binding to Cdc19 amyloids. Nevertheless, breakdown of trehalose upon 
and further boost FBP and ATP production [58]. Finally, high ATP levels may also directly function as biological hydrotrope, and together with increasing cytosolic pH facilitate SG solubilisation [27]. cell is coupled to SG disassembly via the regulated formation of Cdc19 amyloids. With this work, we highlight the crucial function of reversible Cdc19 amyloids in SG regulation and cell survival under stress, and elucidate how functional amyloids can be disassembled in a physiological context. As several metabolic enzymes reversibly aggregate during stress [59], similar mechanisms may regulate their activity. Finally, although the interplay between physiological and pathological amyloids remains to be explored, understanding how amyloids can be regulated in a cellular context might provide important insights into understanding the pathogenesis of neurodegenerative disease.

\section{Methods}

Media, growth assays, yeast strains and plasmid construction

All plasmids and yeast strains used in this work are listed in Supplementary Tables S1 and S2, respectively. Yeast strains and plasmids were obtained using standard molecular biology protocols, and details are available upon request. Yeast cells were grown at $30{ }^{\circ} \mathrm{C}$ in synthetic SD media $(2 \%$ glucose, $0.5 \% \mathrm{NH}_{4}$-sulfate, $0.17 \%$ yeast nitrogen base, and amino acids) or YPD media (2\% C-source, $2 \%$ peptone, $1 \%$ yeast extract), as previously described [4]. Where indicated, $2 \%$ glucose in SD media was substituted with $2 \%$ ethanol. When grown exponentially, $\mathrm{Cdc} 19^{\text {irrev }}$ strains were never allowed to grow above an optical density $600\left(\mathrm{OD}_{600}\right)$ of 0.8 to avoid stress conditions. Heat shock was induced by shifting exponentially growing cells $\left(\mathrm{OD}_{600} 0.4-0.6\right)$ for $30 \mathrm{~min}$ to $42{ }^{\circ} \mathrm{C}$. Wherever indicated, cycloheximide (Sigma-Aldrich, C-7698), was added to the medium to a final concentration of $25 \mu \mathrm{g} / \mathrm{ml}$. Growth rates of different strains before or after stress were determined by measuring $\mathrm{OD}_{600}$ in a $96-$-well plate (Greiner Bio-One) using a plate reader (BioLector m2p-labs or ClarioStar BMG Labtech) over several hours at $30{ }^{\circ} \mathrm{C}$, or by spotting dilution series of those cells onto appropriate SD plates and incubating them for 3 days at $30{ }^{\circ} \mathrm{C}$. Overexpression of proteins from estradiol-inducible promoters was 
achieved by exposing cells to $10 \mathrm{mM}$ estradiol (Sigma-Aldrich E8875) for 3 hours, and Hsp104 activity was inhibited with $5 \mathrm{mM} \mathrm{Gdn-HCl} \mathrm{(Sigma-Aldrich} \mathrm{50950)} \mathrm{for} 3 \mathrm{~h}$ prior to stress initiation.

\section{Fluorescence microscopy, image analysis and quantification} Manager software. For time-lapse experiments, cells were loaded in commercial microfluidic chips (CellASIC ONIX2, Merck Millipore) at $30^{\circ} \mathrm{C}$ as previously described [4]. Heat shock was induced thanks to the chip's temperature controller by increasing the temperature to $42{ }^{\circ} \mathrm{C}$ for $30 \mathrm{~min}$, before returning it to $30{ }^{\circ} \mathrm{C}$, or by shifting cultures for $30 \mathrm{~min}$ in a shaking incubator (Kühner shaker, LT W Lab-Therm) pre-heated at $42{ }^{\circ} \mathrm{C}$ (the two heat shock methods proved to be equivalent). Images were recorded every $10 \mathrm{~min}$. Quantifications were carried out as described in the corresponding figure legends.

\section{Screen for suppressors of irreversible Cdc19 aggregates}

Cells expressing Cdc19 $9^{\text {irrev }}$-GFP were grown in SD-His media to $\mathrm{OD}_{600}$ of 0.6 , heat shocked for 30 min at $42{ }^{\circ} \mathrm{C}$ in a shaking incubator (Kühner shaker, LT W Lab-Therm), collected by centrifugation and spread on SD-His plates to single colonies. Surviving single colonies were isolated and visually inspected by fluorescence microscopy. Pyk2 was deleted by homologous recombination in promising candidates to exclude suppressors that upregulate Pyk2. Remaining suppressors were subsequently backcrossed to wild-type and dissected to ensure Mendelian segregation of suppressors. Suppressor strains with single gene traits were further characterized and subjected to whole-genome sequencing to identify relevant mutations. For this, 10 HIS positive heat shock-resistant spores and 10 HIS positive heat shock-sensitive spores were pooled to minimize sequencing errors and secondary mutations. Identified mutations in single genes were validated by crossing $c d c 19^{\text {irrev }}$ strains with the full-deletion of the mutated gene.

\section{Protein purification}


with $30 \mu \mathrm{g} / \mathrm{ml}$ chloramphenicol and $100 \mu \mathrm{g} / \mathrm{ml}$ carbenicillin to $\mathrm{OD}_{600}$ 0.6. Protein production was induced by adding $0.1 \mathrm{mM}$ IPTG to the cells, which were then grown at $16^{\circ} \mathrm{C}$ overnight. Cells were harvested by centrifugation, resuspended in pre-cooled lysis buffer $(100 \mathrm{mM}$ Tris/HCl pH 7.4, $200 \mathrm{mM}$ $\mathrm{NaCl}, 1 \mathrm{mM} \mathrm{MgCl} 2,10 \%$ glycerol, $1 \mathrm{mM}$ DTT, 1 mM phenylmethylsulfonyl fluoride (PMSF), protease inhibitor mix (Roche) and $75 \mathrm{U} / \mathrm{ml}$ of Pierce universal nuclease), and processed for lysis by freezer milling (SPEX SamplePrep 6870 Freezer/Mill; five cycles of 2 min cooling and 2 min grinding at setting $15 \mathrm{CPS}$ ). Extracts were cleared by centrifugation at $4{ }^{\circ} \mathrm{C}$ for $30 \mathrm{~min}$ at $48000 \mathrm{~g}$, and the supernatant was loaded on a Strep-Tactin Superflow Plus column (Qiagen) at $4{ }^{\circ} \mathrm{C}$ following the manufacturer's instructions. Proteins were eluted using $2.5 \mathrm{mM}$ D-desthiobiotin in purification buffer $(100 \mathrm{mM}$ Tris/ $\mathrm{HCl} \mathrm{pH}$ 7.4, $200 \mathrm{mM} \mathrm{NaCl}, 1 \mathrm{mM} \mathrm{MgCl}$, 10\% glycerol, $1 \mathrm{mM}$ DTT, $1 \mathrm{mM}$ PMSF). Fractions were collected, their purity was checked by SDS-PAGE and Coomassie blue staining, and pure fractions were pooled and aliquoted for storage at $-80^{\circ} \mathrm{C}$ until further use.

\section{Transmission electron microscopy (TEM)}

After thawing on ice, Cdc19 samples in purification buffer $\mathrm{pH} 7.4$ were centrifuged at $21000 \mathrm{~g}$ and $4{ }^{\circ} \mathrm{C}$ for $10 \mathrm{~min}$ and the supernatant with soluble Cdc19 was retrieved. A $0.2 \mathrm{mg} / \mathrm{ml} \mathrm{Cdc} 19$ dilution was then prepared in purification buffer $\mathrm{pH} 7.4$ and the $\mathrm{pH}$ was adjusted to 6-7. Subsequently, a nonglow discharged carbon film 300 mesh copper grid (CF300-CU from Electron Microscopy Sciences) was submerged in this Cdc19 dilution and incubated for $10 \mathrm{~min}$ at $42{ }^{\circ} \mathrm{C}$. Excess sample was then manually removed with Whatman filter paper. Where indicated, grids with pre-formed Cdc19 aggregates were incubated with $4 \mathrm{mM}$ FBP or $4 \mathrm{mM}$ F6P dissolved in purification buffer for $20 \mathrm{~min}$, or simply washed twice with purification buffer, and stained with two drops of $2 \%$ uranyl acetate. Transmission electron micrographs were acquired with a FEI Morgagni 268 microscope at $100 \mathrm{kV}$ using a CCD $1376 \times 1032$ pixel camera at different magnifications.

\section{In vitro protein-metabolite precipitation}

Purified wild-type or mutant $\mathrm{Cdc} 19$ was thawed on ice and cleared by centrifugation $\left(4{ }^{\circ} \mathrm{C}, 10\right.$ min, $21000 \mathrm{~g}$ ). Different metabolites (FBP, F6P ATP, PEP, trehalose) were first solubilized in the same 
buffer as the protein $(100 \mathrm{mM}$ Tris/ $\mathrm{HCl} \mathrm{pH} 7.4,200 \mathrm{mM} \mathrm{NaCl}, 1 \mathrm{mM} \mathrm{MgCl} 2,10 \%$ glycerol, $1 \mathrm{mM}$ DTT, $1 \mathrm{mM}$ PMSF) and were then individually added to Cdc19 to the indicated final concentrations. Final protein concentration was $0.5 \mathrm{mg} / \mathrm{ml}$. Protein-metabolite samples were incubated for $14 \mathrm{~h}$ at 30 ${ }^{\circ} \mathrm{C}$. Aggregated Cdc19 was pelleted by centrifugation $\left(4{ }^{\circ} \mathrm{C}, 10 \mathrm{~min}, 21000 \mathrm{~g}\right)$, and separated from the supernatant containing soluble Cdc19. Aggregation was quantified by loading pellet and supernatant on a SDS-PAGE gel, followed by Coomassie blue staining and quantification with ImageJ software.

\section{In vitro aggregate re-solubilization with FBP}

Purified wild-type $\mathrm{Cdc} 19$ was heat shocked for $10 \mathrm{~min}$ at $42{ }^{\circ} \mathrm{C}$ and the resulting aggregates were incubated with buffer or $20 \mathrm{mM}$ FBP for $5 \mathrm{~h}$ at $4{ }^{\circ} \mathrm{C}$. Aggregates were pelleted by centrifugation $\left(4{ }^{\circ} \mathrm{C}, 10 \mathrm{~min}, 21000 \mathrm{~g}\right.$ ) and $\mathrm{Cdc} 19$ re-solubilization in the presence or absence of FBP was quantified by loading supernatant and pellet fractions on a SDS-PAGE gel, followed by Coomassie blue staining.

\section{Metabolite extraction and LC-MS/MS measurements}

For metabolite extraction, $1 \mathrm{ml}$ culture sample was vacuum-filtered on a $0.45 \mu \mathrm{m}$ pore size PVDF filter (Millipore) and the filter containing the cell pellet was immediately transferred into $4 \mathrm{ml}$ of $-20{ }^{\circ} \mathrm{C}$ cold extraction solution [40:40:20 acetonitrile/methanol/water, HPLC-grade solvents] supplemented with $200 \mathrm{ul}$ of ${ }^{13} \mathrm{C}$ internal standard and incubated at $-20{ }^{\circ} \mathrm{C}$ for $2 \mathrm{~h}$. Afterwards, the extraction solution was transferred to $15 \mathrm{ml}$ tubes on ice, centrifuged for $15 \mathrm{~min}$ at $0{ }^{\circ} \mathrm{C}, 4000 \mathrm{rpm}$ and the supernatant containing the metabolites was transferred to fresh tubes. Samples were dried in a speedvac overnight and stored at $-80^{\circ} \mathrm{C}$ until analysis. Prior to targeted metabolomics measurements, samples were resuspended in $100 \mu \mathrm{lddH_{2 }} \mathrm{O}$.

Compounds were analyzed using an LC-MS/MS mass spectrometer system consisting of a 1290 Infinity LC (Agilent Technologies) coupled to a 5500 QTRAP triple quadrupole mass spectrometer (AB Sciex) in negative multiple reaction monitoring (MRM) scan mode. Five $\mu$ l of metabolite extracts were injected on an Agilent PoroShell 120 HILIC-Z column $(150 \times 2.1 \mathrm{~mm}, 2.7 \mu \mathrm{m}$; Agilent, Santa Clara, CA) using a mobile phase A (water, $10 \mathrm{mM}$ ammonium acetate, $5 \mu \mathrm{M}$ medronic acid, $\mathrm{pH}$ 9) and mobile phase B (90\% acetonitrile, $10 \%$ water, $10 \mathrm{mM}$ ammonium acetate, $5 \mu \mathrm{M}$ medronic acid, $\mathrm{pH}$ 9) 
at a constant flow rate of $250 \mu \mathrm{l} / \mathrm{min}$. Gradient was as follows: 10\% A, 2 min: 10\% A, 12 min: $40 \%$ A, 15 min: 40\% A, 16 min: 10\% A, 24 min: 10\% A. The MRM settings were adapted from Yuan et al. [60]. The raw data were processed and analyzed using custom software in Matlab (Mathworks).

Intracellular metabolite concentration was calculated based on the measured ${ }^{12} \mathrm{C} /{ }^{13} \mathrm{C}$ ratio, assuming a dry weight [61] of $0.53 \mathrm{~g} * 1-1 * \mathrm{OD}-1$ and a cell volume [62] of $0.0021 * \mathrm{~g}-1$. Calibration curves were obtained from dilutions of pure compounds containing same amounts of ${ }^{13} \mathrm{C}$ internal standard.

\section{Protein extraction and Western blotting}

Proteins were precipitated using a TCA/acetone-based protocol. Briefly, exponentially growing cells were incubated $10 \mathrm{~min}$ on ice after the addition of $10 \% \mathrm{TCA}$, centrifuged $\left(5 \mathrm{~min}, 4^{\circ} \mathrm{C}, 21000 \mathrm{~g}\right)$, and pellets were washed twice with acetone. Precipitated proteins were resuspended using a SDS-based loading buffer and protein levels were quantified by Western blotting with specific antibodies. The following antibodies were used (all at 1:3000 dilution): $\alpha$-GFP (Roche, 11814460 001), rabbit polyclonal $\alpha$-Crm1 antibody (provided by Karsten Weis), $\alpha$-Pgk1 (Invitrogen, 459250), HRP-coupled secondary antibody (Biorad, 170-6516) and $\alpha-$ Hsp104 (1:1000) (Abcam, ab69549).

\section{Pyruvate kinase activity assay}

Pyruvate kinase activity was measured with an enzymatic assay where pyruvate production by Cdc19 is coupled to lactate production by lactate dehydrogenase and the simultaneous conversion of $\mathrm{NADH}$ to NAD+ is measured spectrophotometrically at $340 \mathrm{~nm}$. Briefly, purified Cdc19 was diluted to $0.2 \mathrm{mg} / \mathrm{ml}$ and either kept on ice or heat shocked $60 \mathrm{~min}$ at $42{ }^{\circ} \mathrm{C}$. Soluble or aggregated Cdc19 was diluted in an activity buffer $(50 \mathrm{mM}$ imidazole $\mathrm{pH}$ 7, $100 \mathrm{mM} \mathrm{KCl}, 25 \mathrm{mM} \mathrm{MgCl} 2,10 \mathrm{mM}$ ADP, 0.3 $\mathrm{mM}$ NADH, $10 \mathrm{U} / \mathrm{ml} \mathrm{LDH}$ ) to a final protein concentration of $2 \mu \mathrm{g} / \mathrm{ml}$. Reactions were started by adding PEP to a final concentration of $2 \mathrm{mM}$, and decrease in absorbance at $340 \mathrm{~nm}$ was monitored over time.

\section{Size-exclusion chromatography (SEC)}



centrifuging $10 \mathrm{~min}$ at $21000 \mathrm{~g}$ at $4{ }^{\circ} \mathrm{C}$. Protein $(0.1 \mathrm{mg})$ was loaded on a Superdex 200 10/300 GL sizeexclusion column (GE Healthcare) connected to an ÄKTA pure (GE Healthcare) at $4{ }^{\circ} \mathrm{C}$. UV absorbance (280 and $215 \mathrm{~nm}$ ) was measured to follow elution of proteins as a function of elution volume, and fractions were collected, run on a SDS-PAGE gel, and stained with Coomassie blue. type or mutant Cdc19-GFP in SD media to an $\mathrm{OD}_{600}$ of 0.6. Cells were harvested by centrifugation, and pellets were resuspended in lysis buffer (PBS buffer (pH 7.4), 10\% glycerol, 0.5\% Tween 20, $20 \mathrm{mM}$ $\beta$-glycero-phosphate, $1 \mathrm{mM}$ vanadate, $1 \mathrm{mM} \mathrm{NaF}, 1 \mathrm{mM}$ PMSF, protease inhibitor mix (Roche)). Cells were mixed with glass beads (Sigma-Aldrich, 425-600 $\mu \mathrm{m}$ ) and lysed with a FastPrep homogenizer (FastPrep-24 5G, MP Biochemicals). Resulting extracts were cleared first by centrifugation (10 min at $21000 \mathrm{~g}$ at $\left.4{ }^{\circ} \mathrm{C}\right)$, and then by filtering $(0.2 \mu \mathrm{m}$ filter, Millipore). Protein concentration of the supernatant was measured by Nanodrop and a total of 4 mg protein was loaded on a Superdex 200 10/300 GL sizeexclusion column (GE Healthcare) connected to an ÄKTA pure (GE Healthcare). The column had been previously equilibrated in lysis buffer and run according to manufacturer's instructions. Fractions were collected and analyzed by Western blotting with an $\alpha$-GFP antibody.

\section{Circular dichroism (CD), Thioflavin T (ThT) and Congo Red (CR) staining in vitro} spectropolarimeter (Jasco) using a quartz cuvette with $0.1 \mathrm{~cm}$ path length. or Congo Red (CR, Sigma-Aldrich, 75768-25MG) were dissolved in purification buffer (100 mM Tris/ $\mathrm{HCl} \mathrm{pH} 7.4,200 \mathrm{mM} \mathrm{NaCl}, 1 \mathrm{mM} \mathrm{MgCl} 2,10 \%$ glycerol, $1 \mathrm{mM} \mathrm{DTT}, 1 \mathrm{mM}$ PMSF) to a final concentration of $2.5 \mathrm{mM}$ or $1 \mathrm{mM}$, respectively, and filtered ( $0.2 \mu \mathrm{m}$, Millipore). Purified wild-type or mutant $\mathrm{Cdc} 19$ were thawed on ice, cleared by centrifugation $\left(10 \mathrm{~min}, 4{ }^{\circ} \mathrm{C}, 21000 \mathrm{~g}\right)$, diluted to 
intensity was measured in a 384-well plate (Corning Life Sciences) at the indicated temperature using a CLARIOstar plate reader (BMG Labtech). For ThT, excitation was set at $450 \mathrm{~nm}$, and emission spectra were recorded at $490 \mathrm{~nm}$. For CR, fluorescence intensity was measured at $614 \mathrm{~nm}$ with excitation at $560 \mathrm{~nm}$.

\section{Congo Red (CR) staining ex vivo}

For ex vivo CR staining, cells expressing wild-type or mutant Cdc19-GFP were collected by centrifugation and resuspended in lysis buffer (PBS buffer $\mathrm{pH} 7.4,10 \%$ glycerol, $0.5 \%$ Tween 20, 1 mM PMSF). Glass beads (Sigma-Aldrich, $425-600 \mu \mathrm{m}$ ) were added and lysis was performed at $4{ }^{\circ} \mathrm{C}$ with a FastPrep homogenizer (FastPrep-24 5G, MP Biochemicals). Lysates were cleared by centrifuging 2 min at $2000 \mathrm{rpm}$ at $4{ }^{\circ} \mathrm{C}$, and total protein concentration was determined by Nanodrop. Glass microscope slides were cleaned with consecutive washes of $\mathrm{H}_{2} \mathrm{O}, 100 \%$ ethanol and acetone, and carefully dried. Then, $3 \mathrm{~mm}$-wide stripes of double-sided tape were applied along the length of the glass slides in order to form three channels (approximatively $3 \mathrm{~mm}$-wide), which were then covered with a cover slip. Assembled flow chambers were functionalized by incubating them with a solution of 0.3 $\mathrm{mg} / \mathrm{ml}$ Avidin DN (Vector Laboratories) in T50 buffer (10 mM Tris pH 8.0 and $50 \mathrm{mM} \mathrm{NaCl}$ ) for 20 min, then blocking with $0.1 \%$ puronic F-127 (Sigma-Aldrich) in T50 buffer for 30 min, washing twice with $0.1 \mathrm{mg} / \mathrm{ml}$ BSA in T50 buffer, and incubating with biotinylated G-protein in lysis buffer for 30 min. Finally, $10 \mathrm{nM}$ of anti-GFP antibody in lysis buffer were flushed into the channels and total protein extract was loaded in each channel. Sample was allowed to bind for $10 \mathrm{~min}$ and then washed three times with lysis buffer to remove non-bound excess. For staining, a solution of $250 \mu \mathrm{M} \mathrm{CR}$ in lysis buffer was flushed in the channels, and excess was removed by washing twice with lysis buffer. Fluorescence microscopy was performed as described above.

\section{LiP-MS experiments, SRM assay development and quantification analysis.}

For the in vivo LiP-MS experiment, cells expressing Cdc19 $9^{\mathrm{WT}}$-GFP or Cdc19 $9^{\text {irrev }}$-GFP were grown in SD media and three biological replicates were collected when cells were growing exponentially $\left(\mathrm{OD}_{600} 0.6\right)$ or following entry into stationary phase (two days). Cells were resuspended 
in $100 \mathrm{mM}$ HEPES, $150 \mathrm{mM} \mathrm{KCl}, 1 \mathrm{mM} \mathrm{MgCl} 2, \mathrm{pH} 7.6$ and disrupted by bead beating in the presence of acid-washed glass beads in three consecutive rounds of $30 \mathrm{~s}$ of beating and 4 min of incubation at 4 ${ }^{\circ} \mathrm{C}$. Protein concentration in the yeast extracts was determined by the bicinchoninic acid assay (BCA Protein Assay Kit, Thermo Scientific). Based on the protein concentration determined by the BCA assay, equal amounts of the cell lysates were treated with proteinase $\mathrm{K}$ (Sigma Aldrich) at a concentration lysate:proteases of 100:1 for three min. Then, the protease activity was quenched by heating at $95{ }^{\circ} \mathrm{C}$ and by the addition of 5\% sodium deoxycholate (Sigma Aldrich) as described in [63]. Equal amounts of cell lysates were treated in parallel with vehicle instead of proteinase $\mathrm{K}$ to estimate Cdc19 protein amount variations in the two conditions assayed (exponential growth vs stationary phase). Tris(2-carboxyethyl)phosphine-HCl (TCEP-HCl, Thermo Fisher Scientific) was added to a final concentration of $5 \mathrm{mM}\left(35 \mathrm{~min}, 37^{\circ} \mathrm{C}\right)$ to reduce the disulfide bridges, followed by the alkylation of free cysteine residues with iodoacetamide (Sigma aldrich) at $20 \mathrm{mM}$ final concentration (30 min, room temperature). Peptide and protein fragments were then further digested to completion in denaturing conditions with LysC and trypsin proteases. Before the addition of $\mathrm{LysC}$ and trypsin, the reaction mixtures were diluted with $0.1 \mathrm{M}$ ammonium bicarbonate (Sigma Aldrich) to reach a sodium deoxycholate concentration lower than $1 \%$. First, LysC digestion was performed at an enzyme to substrate (E/S) ratio of $1: 100$ for $4 \mathrm{~h}$ at $37{ }^{\circ} \mathrm{C}$, then trypsin digestion was carried out at an E/S ratio of 1:100, for $16 \mathrm{~h}$ at $37^{\circ} \mathrm{C}$. For the in vitro experiment, limited proteolysis experiments were carried out with recombinantly purified $\mathrm{Cdc} 19^{\mathrm{WT}}$ and $\mathrm{Cdc} 19^{\text {irrev }}$ instead of protein lysates. Aliquots of recombinantly purified $\mathrm{Cdc} 19^{\mathrm{WT}}$ and $\mathrm{Cdc} 19^{\text {irrev }}$ were incubated for 10 min at $25^{\circ} \mathrm{C}$ or $42^{\circ} \mathrm{C}$ in triplicates to induce Cdc19 structural variations just before the limited proteolysis step.

The development and validation of Selected Reaction Monitoring (SRM) assays to measure abundances of Cdc19 was guided by a spectral library generated in-house through a LC-MS/MS analysis of the in vivo yeast samples on a QE-plus (Thermo Fisher scientific) mass spectrometer equipped with a nano-electrospray ion source. On-line chromatographic separation of the peptides was achieved with an Easy-nLC 1000 nano liquid chromatography system (Thermo Fisher scientific) equipped with a $40 \mathrm{~cm}$ fused-silica column (New Objective), packed in-house with Reprosil Pur C18 
651 Aq, $1.9 \mu \mathrm{m}$ beads (Dr Maisch). The peptide mixtures $(\sim 1 \mu \mathrm{g})$ were separated with a linear gradient from $6525 \%$ to $35 \%$ acetonitrile in $120 \mathrm{~min}$. The collected spectra were searched against the S. cerevisiae UniProt 653 reference database with Sorcerer-Sequest (Thermo Electron). Trypsin was set as the digesting protease 654 with the tolerance of two missed cleavages and semi tryptic termini. Carbamidomethylation of cysteines 655 $(+57.0214 \mathrm{Da})$ was defined as a fixed modification, and oxidation of methionine $(+15.99492)$ as a variable modification. Protein identifications were filtered with a false discovery rate of $<1 \%$, calculated on the basis of a target-decoy approach. Proteome discoverer 2.2 (Thermo Fisher scientific) was used to build a consensus spectral library using the LiP-MS in vivo samples. Lab Software, University of Washington), and the 10 most intense transitions consisting of doubly or triply charged precursor ions and singly or doubly charged fragment ions of the $y$ - and b-ion series were experimentally tested in SRM mode to select the most suitable transitions for quantification experiments. Samples were measured on a triple quadrupole/ion-trap mass spectrometer (5500 QTrap, ABSciex) equipped with a nano-electrospray ion source and operated in SRM mode. On-line chromatographic separation of the peptides was achieved with an Eksigent 1D-plus Nano liquid chromatography system equipped with a $15 \mathrm{~cm}$ fused-silica column with $75 \mu \mathrm{m}$ inner diameter, packed in-house with Reprosil Pur C18 Aq, $5 \mu \mathrm{m}$ beads (Dr Maisch). The peptide mixtures ( 1 $\mu \mathrm{g}$ ) were separated with a linear gradient from 5\% to $35 \%$ acetonitrile in $30 \mathrm{~min}$. SRM analysis was conducted with Q1 and Q3 operated at unit resolution $(0.7 \mathrm{~m} / \mathrm{z}$ half-maximum peak width) with a dwell time of $20 \mathrm{~ms}$ and a cycle time $<3.0 \mathrm{~s}$.

SRM-MS data was analyzed with Skyline, and for the final SRM assay retention times were scheduled and at least three transitions per precursor ion of Cdc19 were retained. The identity of each SRM peak was confirmed against the spectral library by matching realigned retention times and relative fragment-ion intensities. 199 transitions matching to $44 \mathrm{Cdc} 19$ precursors were selected for Cdc19 peptide quantification purposes. Integration of peak areas was manually inspected and relative quantification was performed for the Cdc19 peptides in the LiP-MS in vitro and in vivo experiments. 
were normalized using the option "equalize medians" of MSstats. In the LiP-MS in vivo experiment peptide intensities were corrected for variations of Cdc19 protein levels between the exponential and stationary conditions. The normalization factor applied was equivalent to the relative variation of the Cdc19 ${ }^{\mathrm{WT}}$ or $\mathrm{Cdc} 19^{\text {irrev }}$ proteins between the exponential and stationary conditions.

\section{Statistical analysis and data availability}

All shown data are representative results from at least three independent experiments, unless differently specified in the respective figure legend. Whenever possible, mean \pm S.E.M. and individual data points of three or more independent experiments are shown. See figure legends for details.GraphPad Prism was used to analyse and plot data.. Detailed experimental procedures and additional data supporting the findings of this study are available from the corresponding authors upon request.

\section{Acknowledgements}

We thank Charlie Boone (Donnelly Centre, University of Toronto, Canada) for providing strains for chaperone overexpression; Karsten Weis (Institute of Biochemistry, ETH Zürich) for antibodies and yeast strains; Claudine Kraft (Institute for Biochemistry and Molecular Biology, University of Freiburg, Germany) for help with yeast cell semi-permeabilization; Sung-Sik Lee, Lorenzo Garbani Marcantini, Jan Schleicher, and Anastasia Timofiiva for help with microscopy and data analysis; ScopeM and the Functional Genomic Center Zürich (FGCZ) for their technical support; Alicia Smith for critical editing, and Roland Riek, Paolo Arosio, Dan Jarosz, Asim Sengör and members of the Peter lab for helpful discussions and comments on the manuscript. This work was funded by the Synapsis Foundation, ETH Zürich, and the Swiss National Science Foundation. In addition, PP received funding from the European Research Council (ERC-CoG), and the EPIC-XS Consortium.

\section{Author contributions}


AA, IP, HY, SS; Writing - Original Draft Preparation: GC, MP; Writing - Review \& Editing: GC,

705

706

707

708

709

710

711

712

713

714

715

716

717

718

719

720

721

722

723

724

725

726

727

728

729

730

731

732

733

734

735

736

737

738

739

740
MP, with input from all authors; Visualization: GC, MP; Supervision: MP, PP, US, AD; Funding

Acquisition: MP, PP, US, AD. The authors declare that they have no competing financial interest

related to this paper.

\section{References}

1. Fulda, S., et al., Cellular Stress Responses: Cell Survival and Cell Death. International Journal of Cell Biology, 2010. 2010: p. 214074.

2. Aulas, A., et al., Stress-specific differences in assembly and composition of stress granules and related foci. J Cell Sci, 2017. 130(5): p. 927-937.

3. Protter, D.S.W. and R. Parker, Principles and Properties of Stress Granules. Trends Cell Biol, 2016. 26(9): p. 668-679.

4. Saad, S., et al., Reversible protein aggregation is a protective mechanism to ensure cell cycle restart after stress. Nat Cell Biol, 2017. 19(10): p. 1202-1213.

5. Begovich, K., et al., Conserved metabolite regulation of stress granule assembly via AdoMet. J Cell Biol, 2020. 219(8).

6. Cabrera, M., et al., Chaperone-Facilitated Aggregation of Thermo-Sensitive Proteins Shields Them from Degradation during Heat Stress. Cell Rep, 2020. 30(7): p. 2430-2443.e4.

7. Wolozin, B. and P. Ivanov, Stress granules and neurodegeneration. Nat Rev Neurosci, 2019. 20(11): p. 649-666.

8. Cao, X., X. Jin, and B. Liu, The involvement of stress granules in aging and aging-associated diseases. Aging Cell, 2020. 19(4): p. e13136.

9. Jain, S., et al., ATPase-Modulated Stress Granules Contain a Diverse Proteome and Substructure. Cell, 2016. 164(3): p. 487-98.

10. Molliex, A., et al., Phase separation by low complexity domains promotes stress granule assembly and drives pathological fibrillization. Cell, 2015. 163(1): p. 123-33.

11. Banani, S.F., et al., Biomolecular condensates: organizers of cellular biochemistry. Nat Rev Mol Cell Biol, 2017. 18(5): p. 285-298.

12. Van Treeck, B., et al., RNA self-assembly contributes to stress granule formation and defining the stress granule transcriptome. Proc Natl Acad Sci U S A, 2018. 115(11): p. 2734-2739.

13. Hofmann, S., et al., Molecular mechanisms of stress granule assembly and disassembly. Biochim Biophys Acta Mol Cell Res, 2021. 1868(1): p. 118876.

14. Bounedjah, O., et al., Macromolecular crowding regulates assembly of mRNA stress granules after osmotic stress: new role for compatible osmolytes. J Biol Chem, 2012. 287(4): p. 244658.

15. Persson, L.B., V.S. Ambati, and O. Brandman, Cellular Control of Viscosity Counters Changes in Temperature and Energy Availability. Cell, 2020.

16. Ohn, T., et al., A functional RNAi screen links O-GICNAc modification of ribosomal proteins to stress granule and processing body assembly. Nat Cell Biol, 2008. 10(10): p. 1224-31.

17. Eum, H., et al., ATP-driven reactions are required for the assembly of large stress granules. Biochem Biophys Res Commun, 2020. 521(1): p. 238-244.

18. Kroschwald, S., et al., Different Material States of Pub1 Condensates Define Distinct Modes of Stress Adaptation and Recovery. Cell Rep, 2018. 23(11): p. 3327-3339. 
19. Sathyanarayanan, U., et al., ATP hydrolysis by yeast Hsp104 determines protein aggregate dissolution and size in vivo. Nat Commun, 2020. 11(1): p. 5226.

20. Walters, R.W., et al., Differential effects of Ydj1 and Sis1 on Hsp70-mediated clearance of stress granules in Saccharomyces cerevisiae. Rna, 2015. 21(9): p. 1660-71.

21. Buchan, J.R., et al., Eukaryotic stress granules are cleared by autophagy and Cdc48/VCP function. Cell, 2013. 153(7): p. 1461-74.

22. Shattuck, J.E., et al., The prion-like protein kinase Sky1 is required for efficient stress granule disassembly. Nat Commun, 2019. 10(1): p. 3614.

23. Wang, B., et al., ULK1 and ULK2 Regulate Stress Granule Disassembly Through Phosphorylation and Activation of VCP/p97. Mol Cell, 2019. 74(4): p. 742-757.e8.

24. Hondele, M., et al., DEAD-box ATPases are global regulators of phase-separated organelles. Nature, 2019. 573(7772): p. 144-148.

25. Chalupnikova, K., et al., Recruitment of the RNA helicase RHAU to stress granules via a unique RNA-binding domain. J Biol Chem, 2008. 283(50): p. 35186-98.

26. Loschi, M., et al., Dynein and kinesin regulate stress-granule and P-body dynamics. J Cell Sci, 2009. 122(Pt 21): p. 3973-82.

27. Patel, A., et al., ATP as a biological hydrotrope. Science, 2017. 356(6339): p. 753-756.

28. Hofmann, S., et al., Translation suppression promotes stress granule formation and cell survival in response to cold shock. Mol Biol Cell, 2012. 23(19): p. 3786-800.

29. Weitzel, G., U. Pilatus, and L. Rensing, The cytoplasmic $\mathrm{pH}$, ATP content and total protein synthesis rate during heat-shock protein inducing treatments in yeast. Exp Cell Res, 1987. 170(1): p. 64-79.

30. Soini, J., et al., Transient increase of ATP as a response to temperature up-shift in Escherichia coli. Microb Cell Fact, 2005. 4(1): p. 9.

31. Xu, Y.F., et al., Regulation of yeast pyruvate kinase by ultrasensitive allostery independent of phosphorylation. Mol Cell, 2012. 48(1): p. 52-62.

32. Fiechter, A., G.F. Fuhrmann, and O. Käppeli, Regulation of glucose metabolism in growing yeast cells. Adv Microb Physiol, 1981. 22: p. 123-83.

33. Flores, C.L., et al., Carbohydrate and energy-yielding metabolism in non-conventional yeasts. FEMS Microbiol Rev, 2000. 24(4): p. 507-29.

34. Grignaschi, E., et al., A hydrophobic low-complexity region regulates aggregation of the yeast pyruvate kinase Cdc19 into amyloid-like aggregates in vitro. J Biol Chem, 2018. 293(29): p. 11424-11432.

35. Anastasiou, D., et al., Pyruvate kinase M2 activators promote tetramer formation and suppress tumorigenesis. Nat Chem Biol, 2012. 8(10): p. 839-47.

36. Blázquez, M.A., et al., Trehalose-6-phosphate, a new regulator of yeast glycolysis that inhibits hexokinases. FEBS Lett, 1993. 329(1-2): p. 51-4.

37. van Vaeck, C., et al., Analysis and modification of trehalose 6-phosphate levels in the yeast Saccharomyces cerevisiae with the use of Bacillus subtilis phosphotrehalase. Biochem J, 2001. 353(Pt 1): p. 157-162.

38. Peeters, K., et al., Fructose-1,6-bisphosphate couples glycolytic flux to activation of Ras. Nat Commun, 2017. 8(1): p. 922.

39. Cereghetti, G., et al., Reversible, functional amyloids: towards an understanding of their regulation in yeast and humans. Cell Cycle, 2018: p. 1-14.

40. Villar-Piqué, A., et al., Screening for amyloid aggregation: in-silico, in-vitro and in-vivo detection. Curr Protein Pept Sci, 2014. 15(5): p. 477-89.

41. Linder, T., Evaluation of the chitin-binding dye Congo red as a selection agent for the isolation, classification, and enumeration of ascomycete yeasts. Arch Microbiol, 2018. 200(4): p. 671-675.

42. Feng, Y., et al., Global analysis of protein structural changes in complex proteomes. Nat Biotechnol, 2014. 32(10): p. 1036-44. 
43. Cappelletti, V., et al., Dynamic 3D proteomes reveal protein functional alterations at high resolution in situ. Cell, 2021. 184(2): p. 545-559.e22.

44. Boles, E., et al., Characterization of a glucose-repressed pyruvate kinase (Pyk $2 p)$ in Saccharomyces cerevisiae that is catalytically insensitive to fructose-1,6-bisphosphate. J Bacteriol, 1997. 179(9): p. 2987-93.

45. Orlandi, I., et al., Ethanol and acetate acting as carbon/energy sources negatively affect yeast chronological aging. Oxid Med Cell Longev, 2013. 2013: p. 802870.

46. Bell, W., et al., Composition and functional analysis of the Saccharomyces cerevisiae trehalose synthase complex. J Biol Chem, 1998. 273(50): p. 33311-9.

47. Monteiro, F., et al., Measuring glycolytic flux in single yeast cells with an orthogonal synthetic biosensor. Mol Syst Biol, 2019. 15(12): p. e9071.

48. Ji, F., et al., Determination of intracellular metabolites concentrations in Escherichia coli under nutrition stress using liquid chromatography-tandem mass spectrometry. Talanta, 2018. 189: p. 1-7.

49. Fenton, A.W. and J.B. Blair, Kinetic and allosteric consequences of mutations in the subunit and domain interfaces and the allosteric site of yeast pyruvate kinase. Arch Biochem Biophys, 2002. 397(1): p. 28-39.

50. Cherkasov, V., et al., Coordination of translational control and protein homeostasis during severe heat stress. Curr Biol, 2013. 23(24): p. 2452-62.

51. Mogk, A., B. Bukau, and H.H. Kampinga, Cellular Handling of Protein Aggregates by Disaggregation Machines. Mol Cell, 2018. 69(2): p. 214-226.

52. Hanssum, A., et al., An inducible chaperone adapts proteasome assembly to stress. Mol Cell, 2014. 55(4): p. 566-77.

53. Ciechanover, A. and Y.T. Kwon, Degradation of misfolded proteins in neurodegenerative diseases: therapeutic targets and strategies. Exp Mol Med, 2015. 47(3): p. e147.

54. Bolognesi, B., et al., Single point mutations induce a switch in the molecular mechanism of the aggregation of the Alzheimer's disease associated A642 peptide. ACS Chem Biol, 2014. 9(2): p. 378-82.

55. Jin, M., et al., Glycolytic Enzymes Coalesce in G Bodies under Hypoxic Stress. Cell Rep, 2017. 20(4): p. 895-908.

56. Bösl, B., V. Grimminger, and S. Walter, Substrate binding to the molecular chaperone Hsp104 and its regulation by nucleotides. J Biol Chem, 2005. 280(46): p. 38170-6.

57. Gutierres, M.B.B., C.B.C. Bonorino, and M.M. Rigo, ChaperISM: improved chaperone binding prediction using position-independent scoring matrices. Bioinformatics, 2020. 36(3): p. 735741.

58. Suarez-Mendez, C.A., C. Ras, and S.A. Wahl, Metabolic adjustment upon repetitive substrate perturbations using dynamic (13)C-tracing in yeast. Microb Cell Fact, 2017. 16(1): p. 161.

59. Narayanaswamy, R., et al., Widespread reorganization of metabolic enzymes into reversible assemblies upon nutrient starvation. Proc Natl Acad Sci U S A, 2009. 106(25): p. 10147-52.

60. Yuan, M., et al., A positive/negative ion-switching, targeted mass spectrometry-based metabolomics platform for bodily fluids, cells, and fresh and fixed tissue. Nat Protoc, 2012. 7(5): p. 872-81.

61. Sonderegger, M. and U. Sauer, Evolutionary engineering of Saccharomyces cerevisiae for anaerobic growth on xylose. Appl Environ Microbiol, 2003. 69(4): p. 1990-8.

62. Lamprecht, I., B. Schaarschmidt, and G. Welge, Microcalorimetric investigation of the metabolism of yeasts. $V$. Influence of ploidy on growth and metabolism. Radiat Environ Biophys, 1976. 13(1): p. 57-61.

63. Piazza, I., et al., A Map of Protein-Metabolite Interactions Reveals Principles of Chemical Communication. Cell, 2018. 172(1-2): p. 358-372.e23. 
64. Plata, M.R., et al., Determination of carbohydrates present in Saccharomyces cerevisiae using mid-infrared spectroscopy and partial least squares regression. Anal Bioanal Chem, 2013. 405(25): p. 8241-50. 
Figure 1 - SG disassembly depends on re-solubilization of Cdc19 amyloids and restoration of ATP production

A
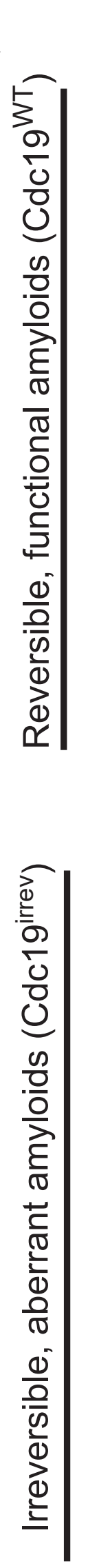

D

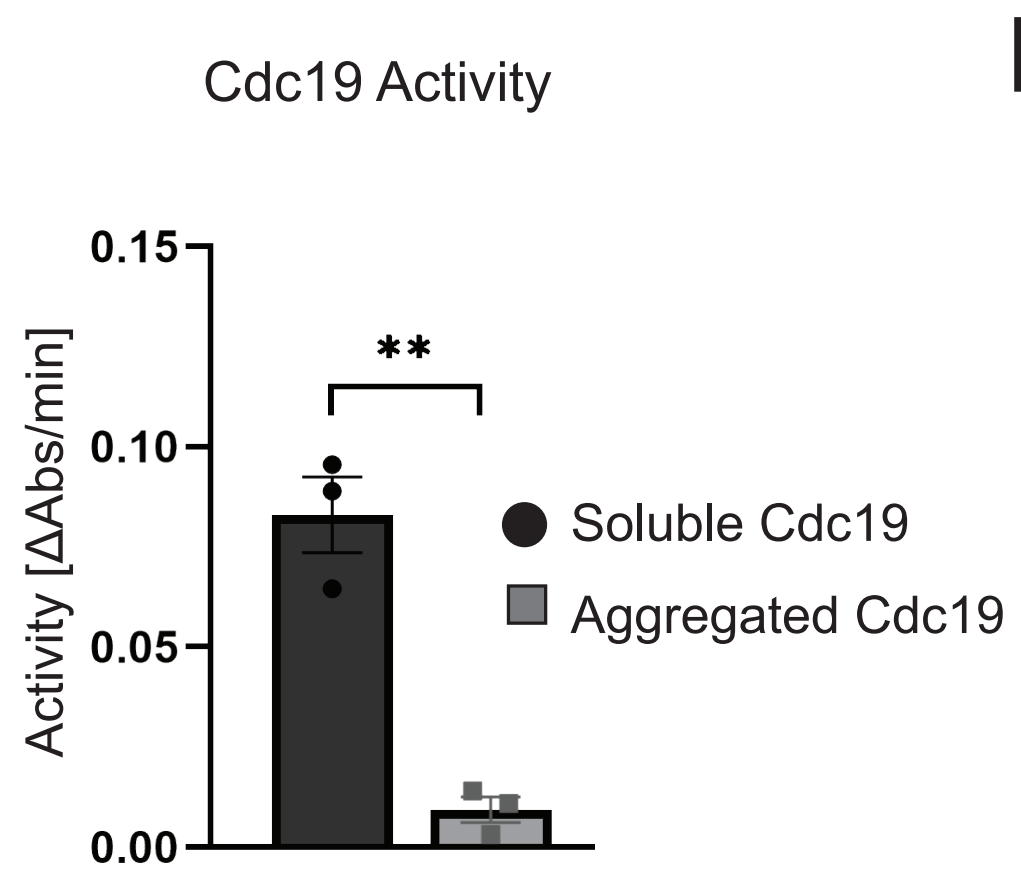

G

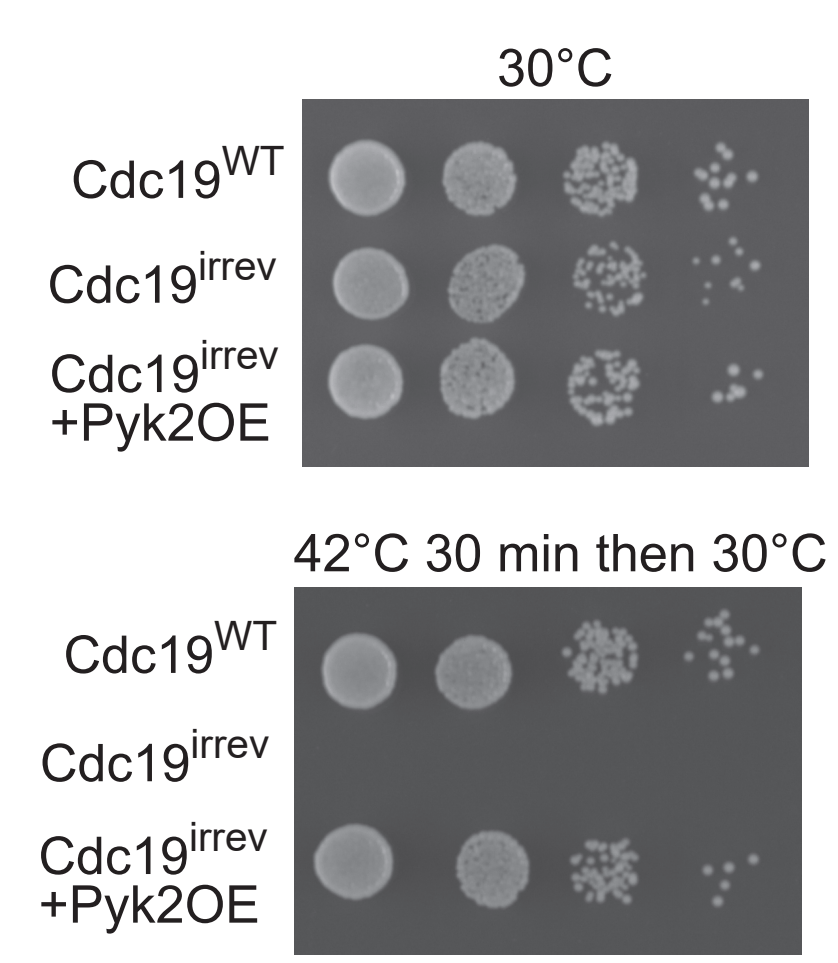

B
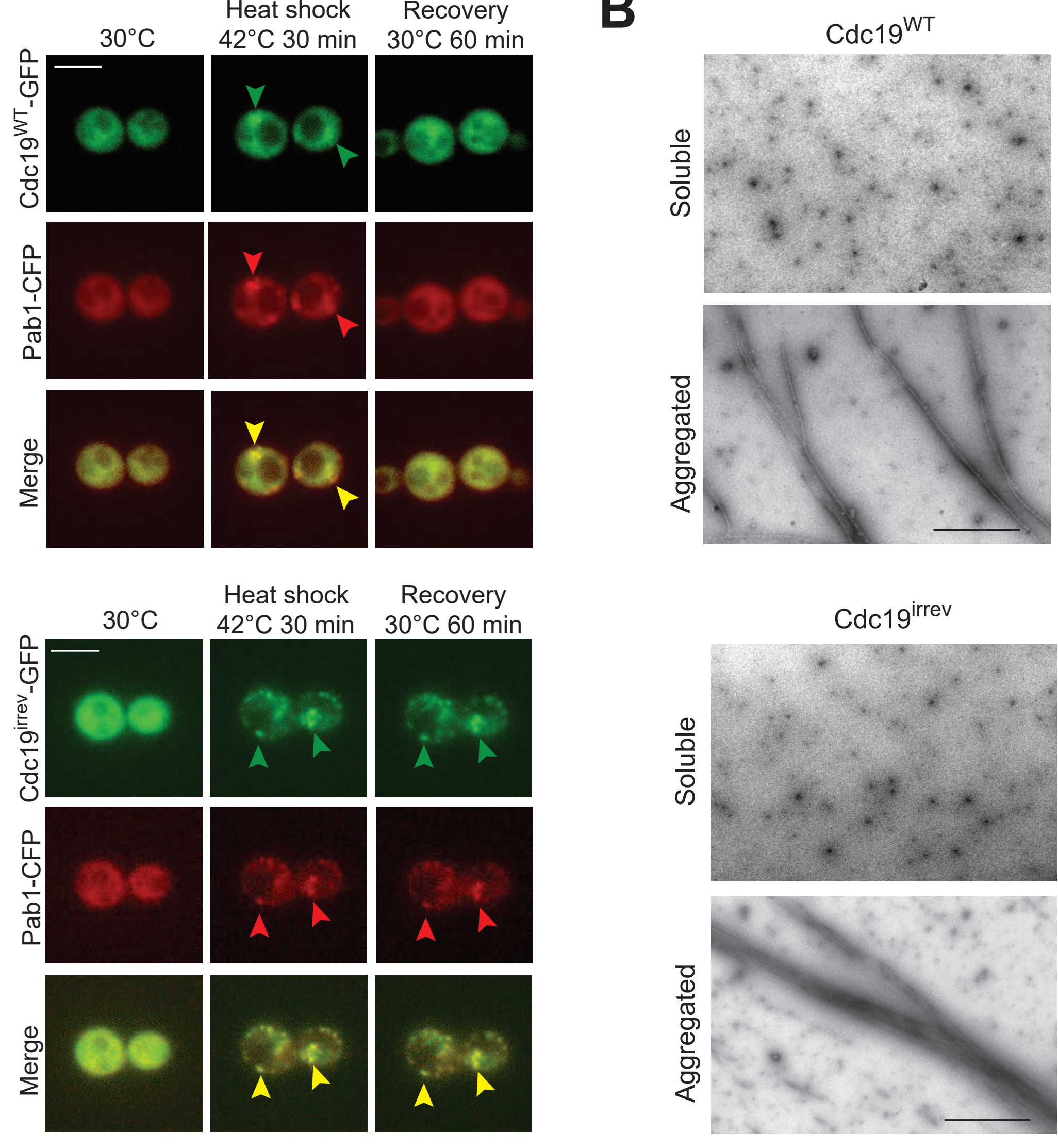

E

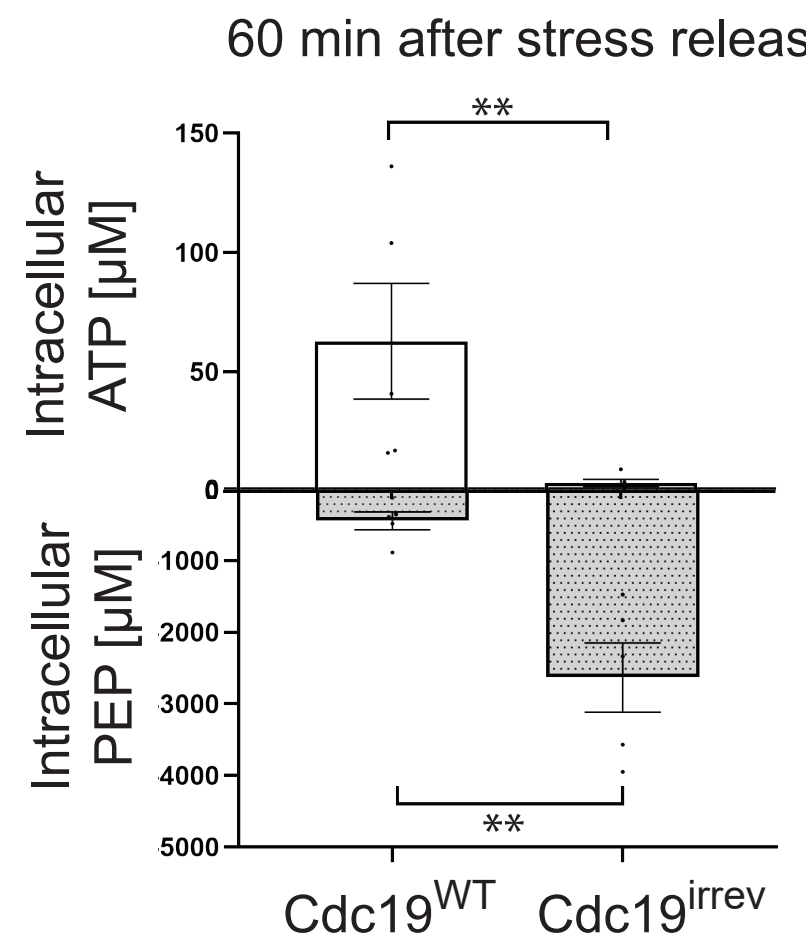

H

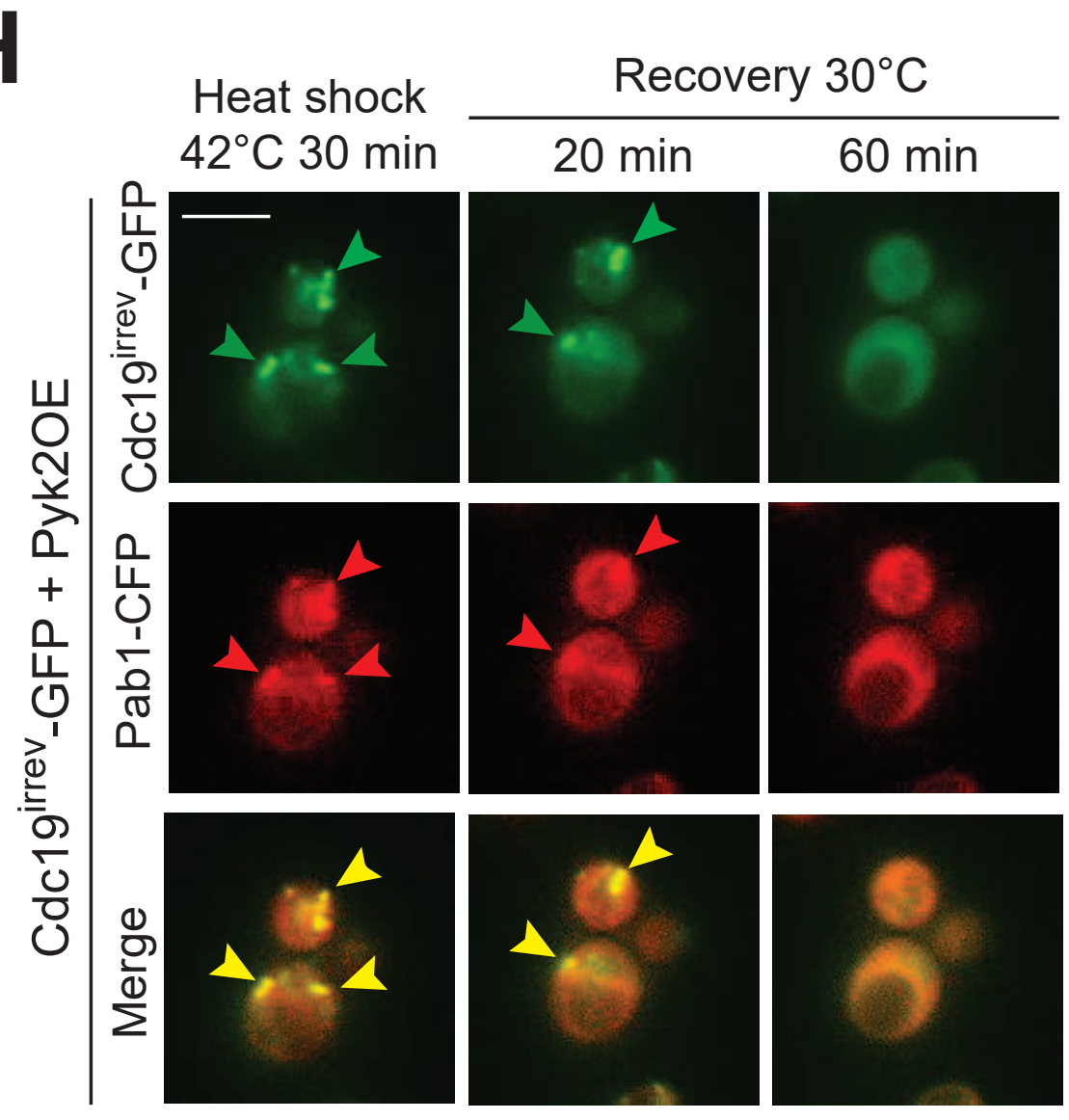

Cdc19 $9^{\text {irrev }}$
C
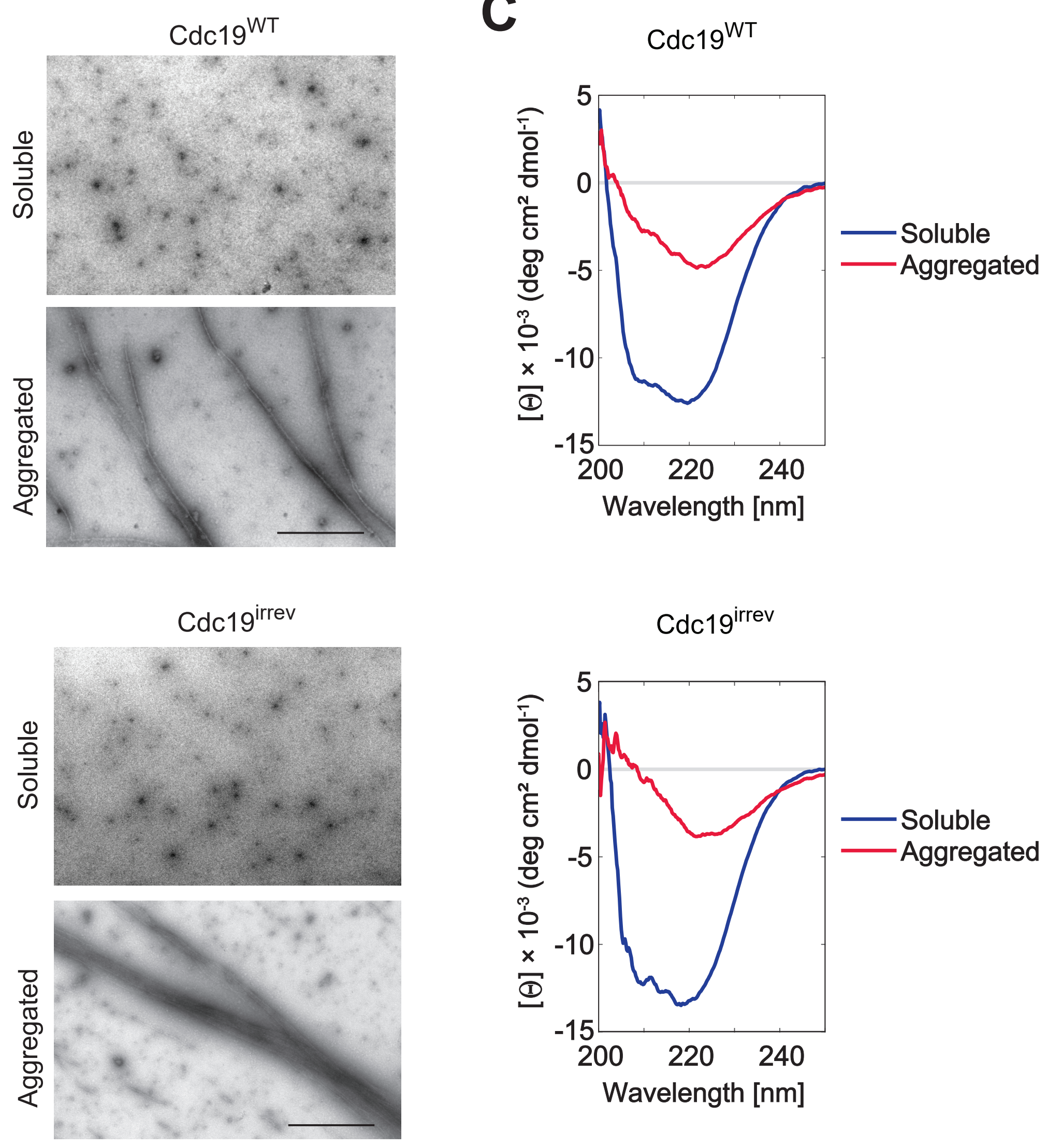

Cdc19irrev

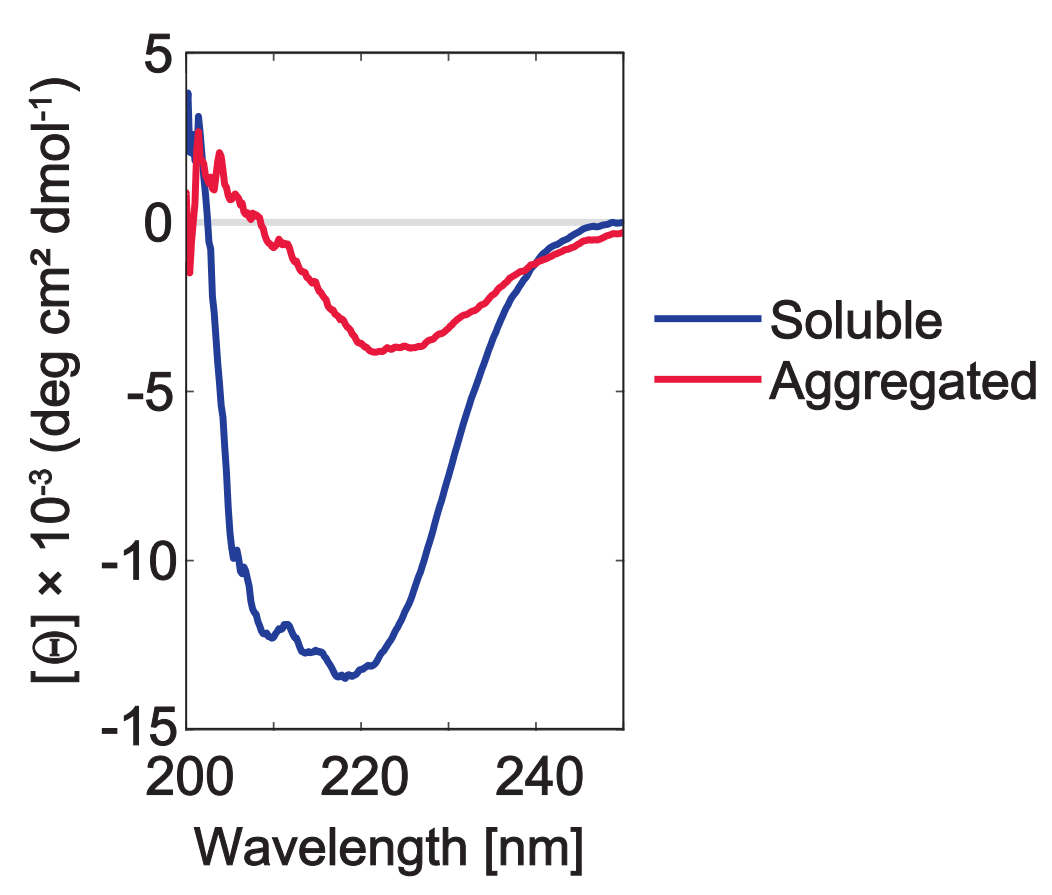

F

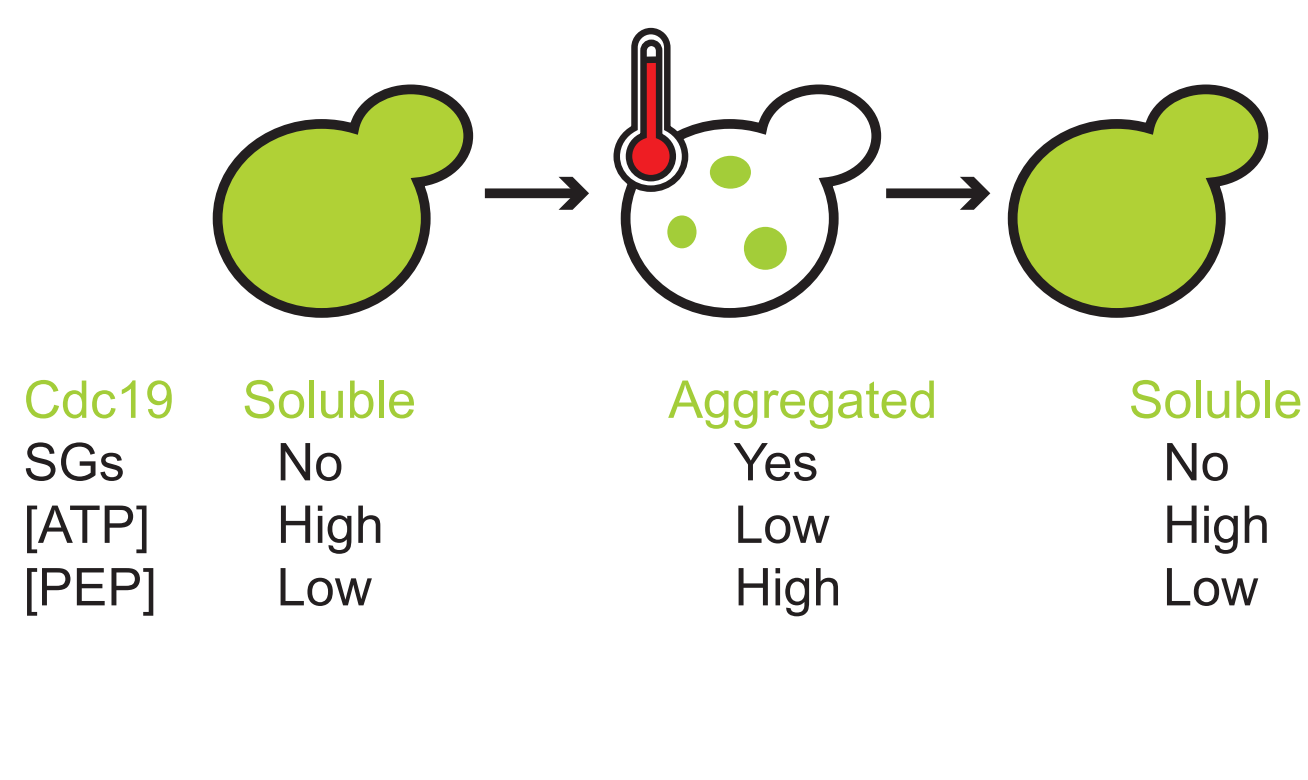

I

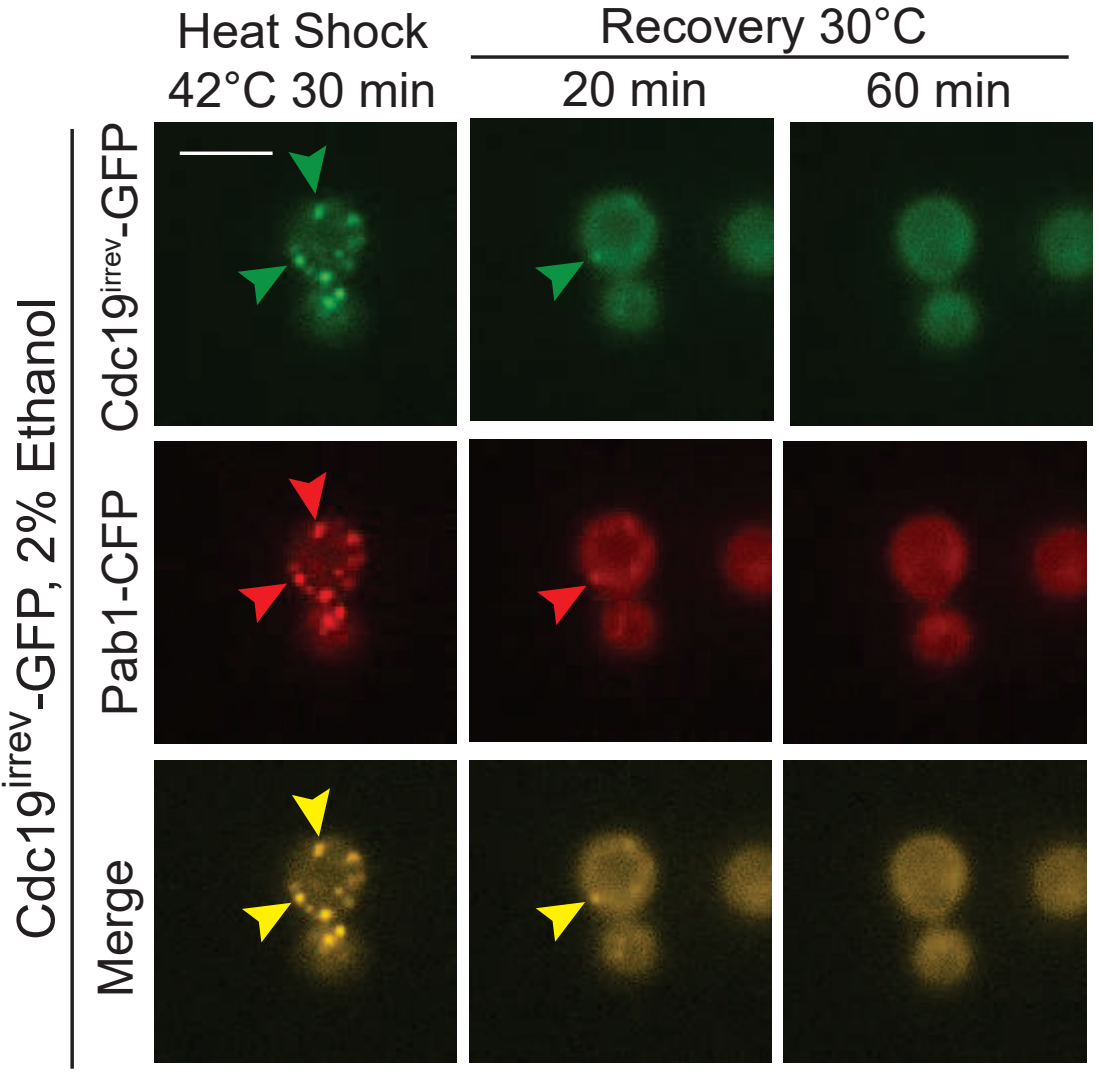


Figure 1: SG disassembly depends on re-solubilization of Cdc19 amyloids and restoration of

\section{ATP production}

(A) Exponentially growing Cdc19 ${ }^{\mathrm{WT}}$-GFP (top panel) and Cdc19 $9^{\text {irrev }}$-GFP (bottom panel) cells expressing the SG marker Pab1-CFP were grown in a microfluidic chip at $30^{\circ} \mathrm{C}$ in SD-full medium (left column), subjected to heat stress for 30 min at $42{ }^{\circ} \mathrm{C}$ (middle column) and allowed to recover at $30{ }^{\circ} \mathrm{C}$ (right column). Representative GFP- (top row) and CFP-images (middle row) were taken at the indicated time points, and merged (bottom row) to show co-localization of Cdc19 aggregates and SGs (arrow heads). Note that upon heat shock, Cdc19 ${ }^{\mathrm{WT}}$-GFP forms reversible aggregates that co-localize with SGs. The mutant Cdc19 $9^{\text {irrev }}$-GFP instead forms irreversible aggregates, which prevent SG disassembly after stress release. Images are representative of three independent experiments. Scale bar: $5 \mu \mathrm{m}$.

(B) - (C) $\mathrm{Cdc1} 19^{\mathrm{WT}}$ (top panels) or Cdc19 $9^{\text {irrev }}$ (bottom panels) proteins were expressed and purified from E. coli, and imaged by negative staining transmission electron microscopy (TEM) (B) or analyzed by circular dichroism (CD) (C) before (soluble) or after a 10 min heat shock at $42{ }^{\circ} \mathrm{C}$ (aggregated). Representative images of three independent experiments are shown. Note that upon heat shock Cdc19 assembles into $\beta$-sheet-rich, filamentous amyloids. Scale bar in (B): 2 $\mu \mathrm{m}$.

(D) The catalytic activity of purified $\mathrm{Cdc} 19^{\mathrm{WT}}$ was measured using a lactate dehydrogenase-coupled activity assay before (soluble) or after a $30 \mathrm{~min}$ heat shock at $42{ }^{\circ} \mathrm{C}$ (aggregated). The bars show Cdc19 activity expressed as the decrease in absorbance at $340 \mathrm{~nm}$ per min. Mean, standard error of the mean (S.E.M.) and single data points of three independent experiments are indicated (statistical analysis with two-tailed t-test, $P=0.0018$ ).

(E) $c d c 19^{\text {irrev }}$ cells are unable to re-activate Cdc19 and resume ATP production after stress release. Intracellular ATP and phosphoenolpyruvate (PEP) concentrations $(\mu \mathrm{M})$ were measured in wildtype and $c d c 19^{\text {irrev }}$ cells, after these cells were subjected to a 30 min heat shock at $42{ }^{\circ} \mathrm{C}$ and allowed to recover at $30{ }^{\circ} \mathrm{C}$ for $60 \mathrm{~min}$. Mean and S.E.M. determined by five independent experiments are shown (statistical analysis with Mann-Whitney test, $P_{A T P}=0.0079, P_{P E P}=$ 

Cdc19, while $c d c 19^{\text {irrev }}$ cells are not able to consume PEP and resume ATP production.

(F) Schematic drawing showing a yeast cell before, during, and after heat stress. Upon stress, Cdc19 (represented in green) forms inactive aggregates, which colocalize with SGs. ATP levels rapidly decrease under these conditions, while Cdc19's substrate phosphoenolpyruvate (PEP) accumulates. Upon stress release, Cdc19 re-solubilizes, gets re-activated, and consumes PEP to produce ATP, concomitant with SG disassembly.

(G) Serial dilutions of exponentially growing cells expressing Cdc19 $9^{\mathrm{WT}}$-GFP or Cdc19 $9^{\text {irrev }}$-GFP and harboring where indicated a plasmid overexpressing Pyk2 from the constitutive TEF-promoter (Pyk2OE) were spotted on agar plates before (upper panel) or after a 30 min heat shock at $42{ }^{\circ} \mathrm{C}$ (lower panel). The plates were incubated at $30{ }^{\circ} \mathrm{C}$ and imaged after 3 days.

(H) - (I) ATP production using Cdc19-independent pathways allows Cdc19 $9^{\text {irrev }}$ aggregate and SG

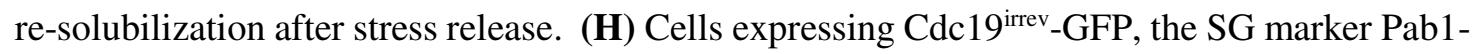
CFP, and a plasmid overexpressing Pyk2 from the constitutive TEF-promoter (Pyk2OE) were grown at $30{ }^{\circ} \mathrm{C}$ in $\mathrm{SD}$-full medium, subjected to heat stress for $30 \mathrm{~min}$ at $42{ }^{\circ} \mathrm{C}$ (left column) and allowed to recover at $30{ }^{\circ} \mathrm{C}$ (middle and right columns) in SD-full medium with $25 \mu \mathrm{g} / \mathrm{ml}$ cycloheximide to avoid de novo protein synthesis. (I) Cells expressing Cdc1 $19^{\text {irrev }}$-GFP and the SG marker Pab1-CFP were grown in SD medium containing $2 \%$ ethanol as the sole carbon source at $30{ }^{\circ} \mathrm{C}$, heat shocked for $30 \mathrm{~min}$ at $42{ }^{\circ} \mathrm{C}$ (left column) and allowed to recover at $30{ }^{\circ} \mathrm{C}$ (middle and right columns) in SD-full medium with $25 \mu \mathrm{g} / \mathrm{ml}$ cycloheximide to avoid de novo protein synthesis. Representative GFP- (top row) and CFP-images (middle row) were taken at 
Figure 2 - Re-solubilization of Cdc19irev amyloids and cell cycle restart after stress are regulated by the glycolytic metabolite FBP

A

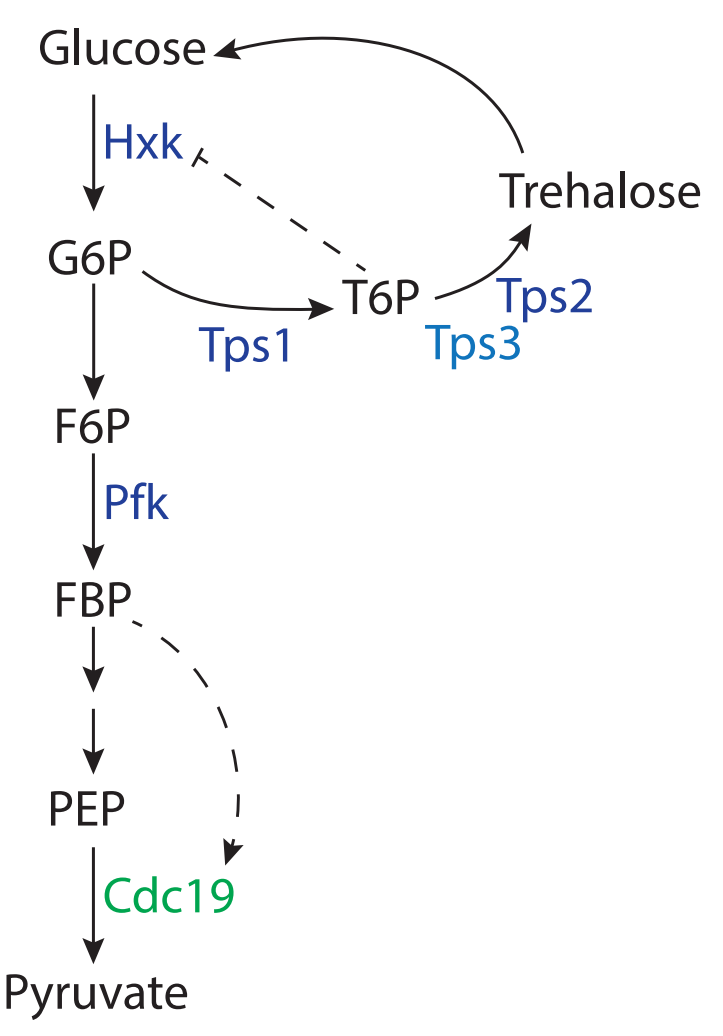

C

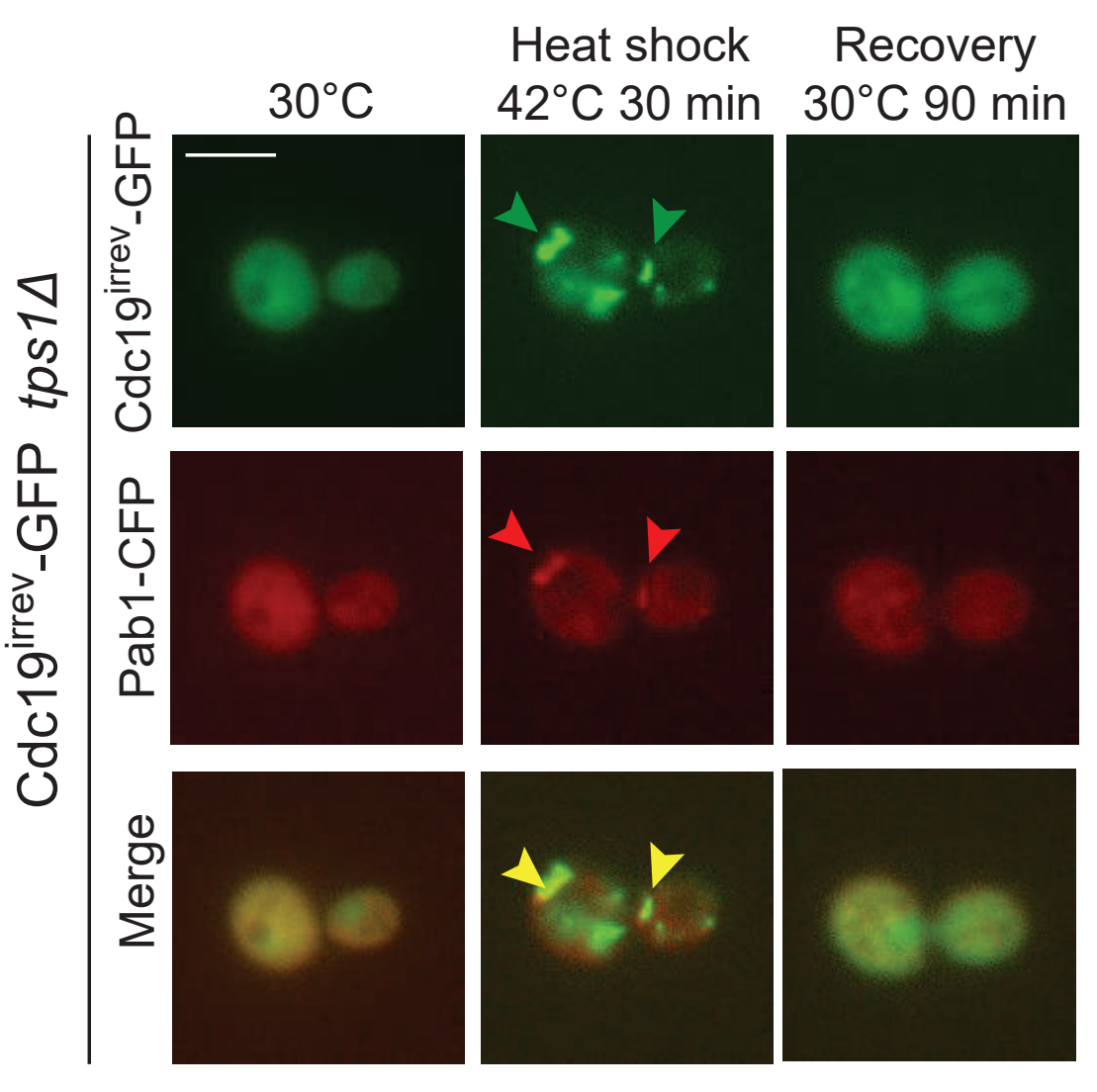

E

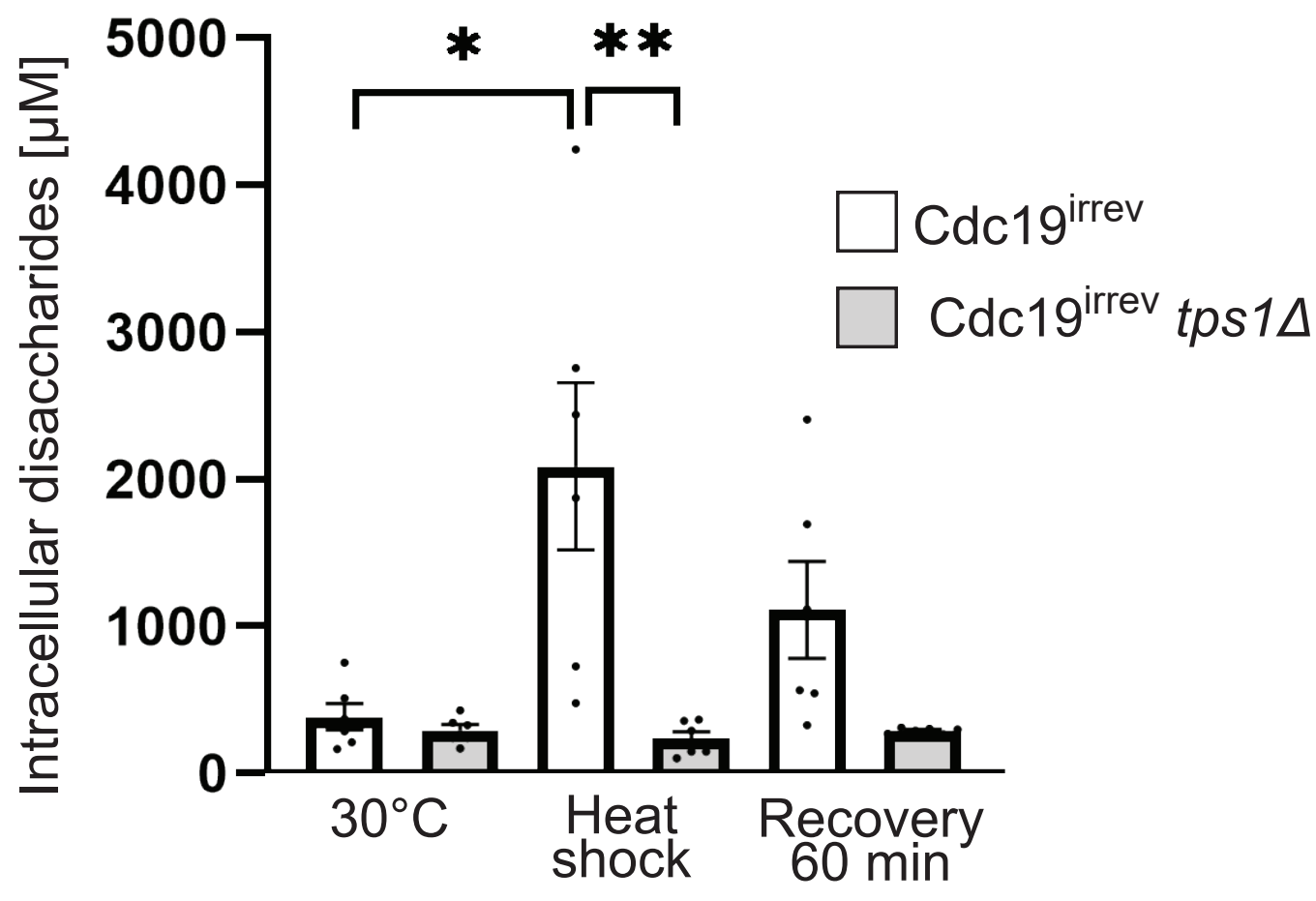

G
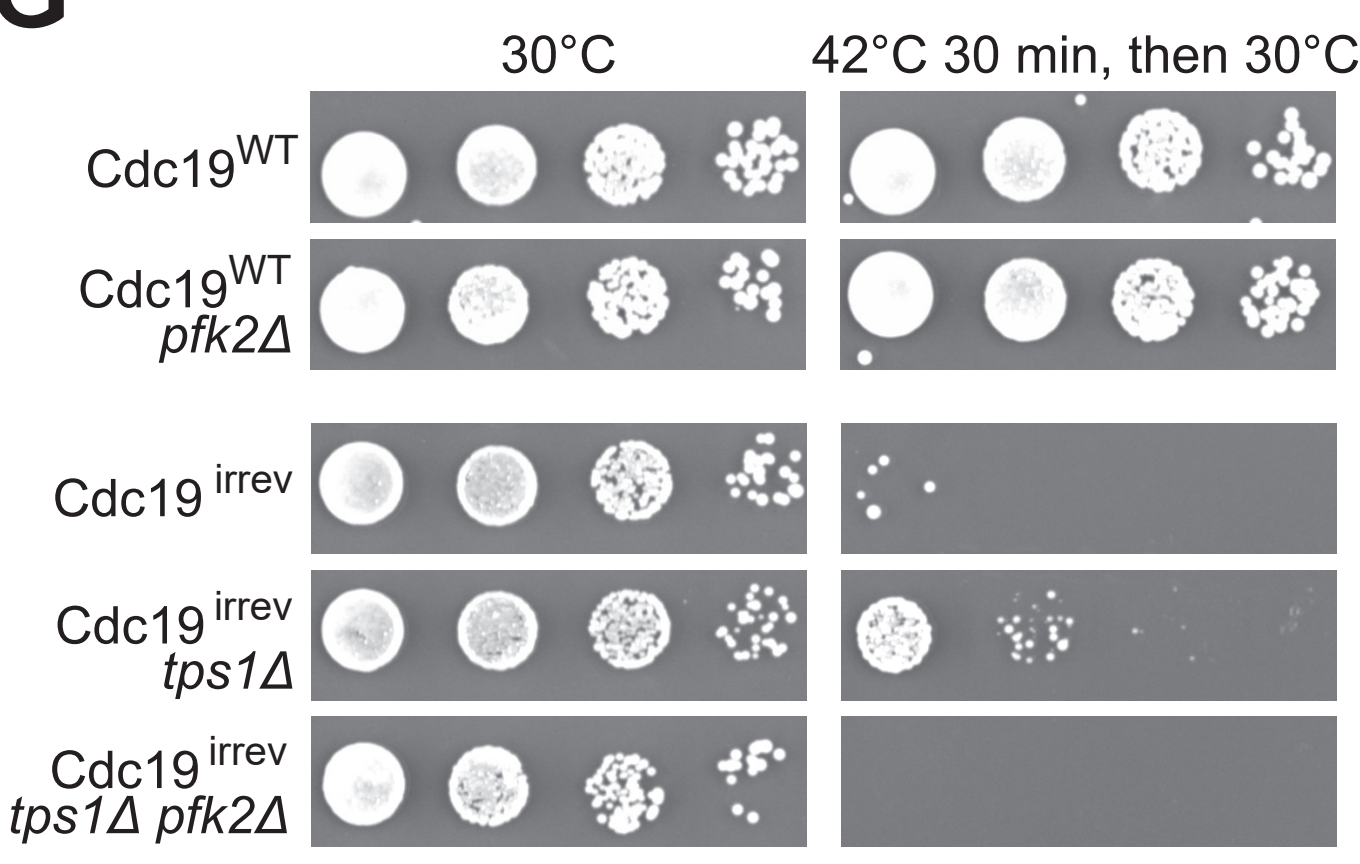

B

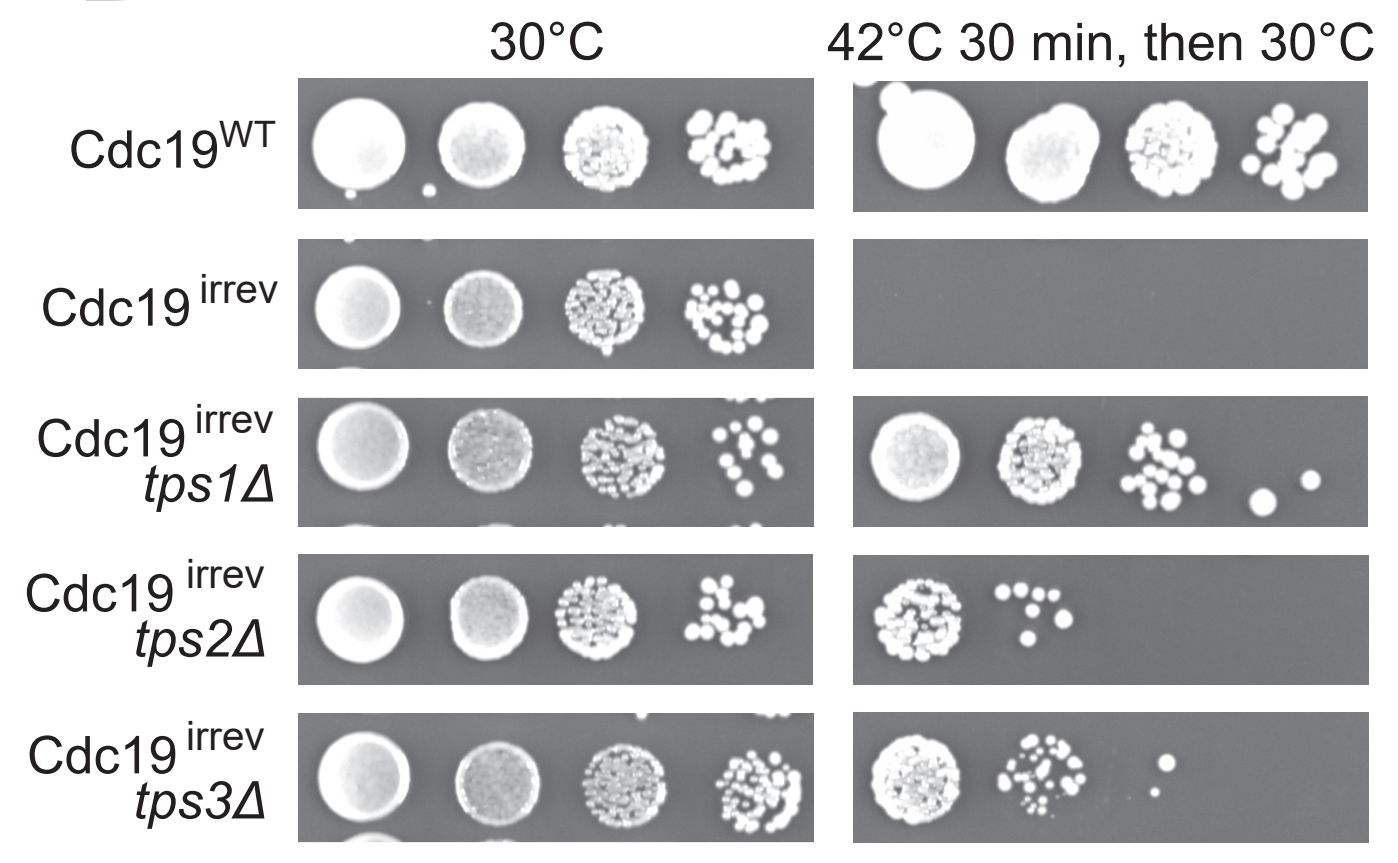

D

60 min after stress release

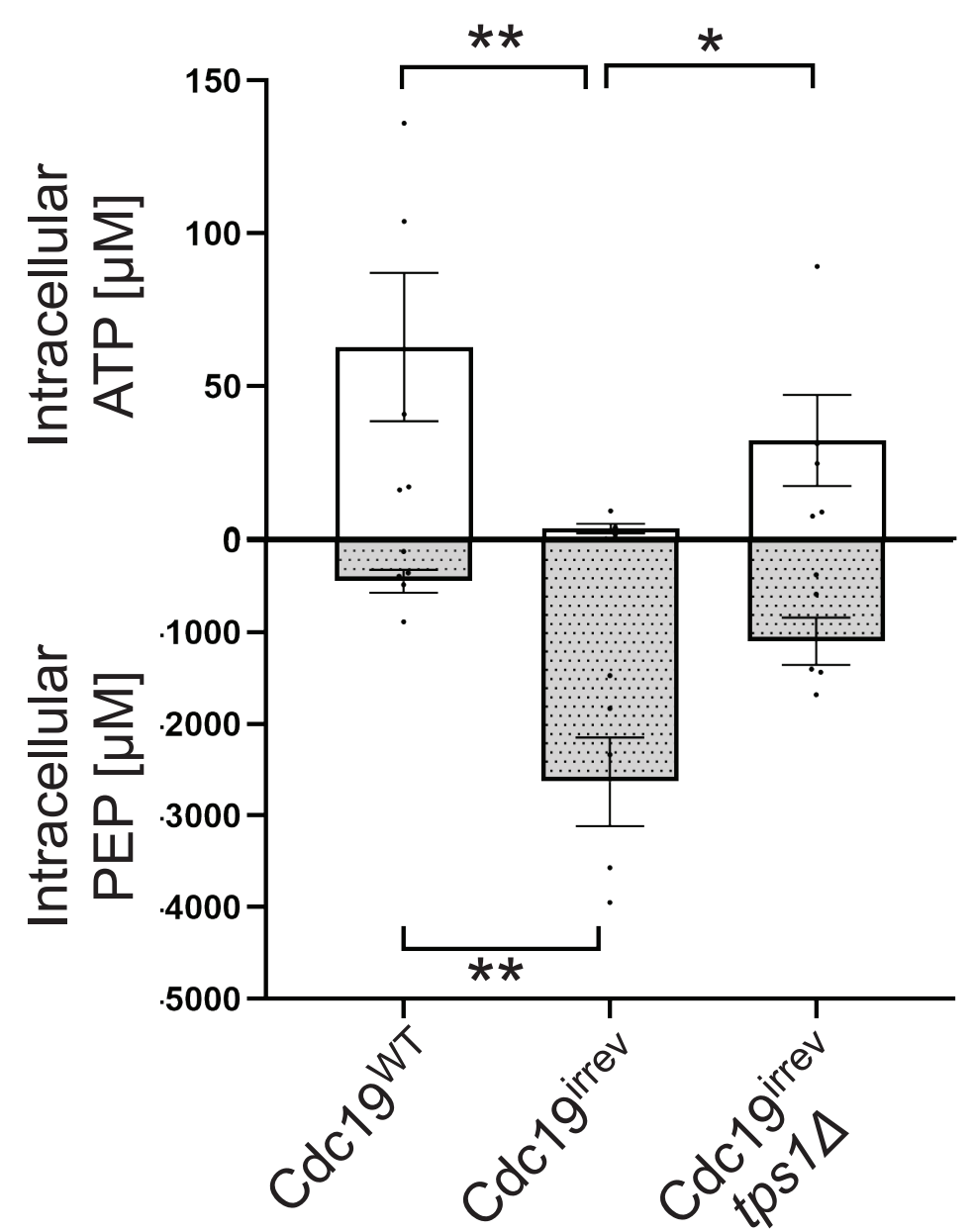

F
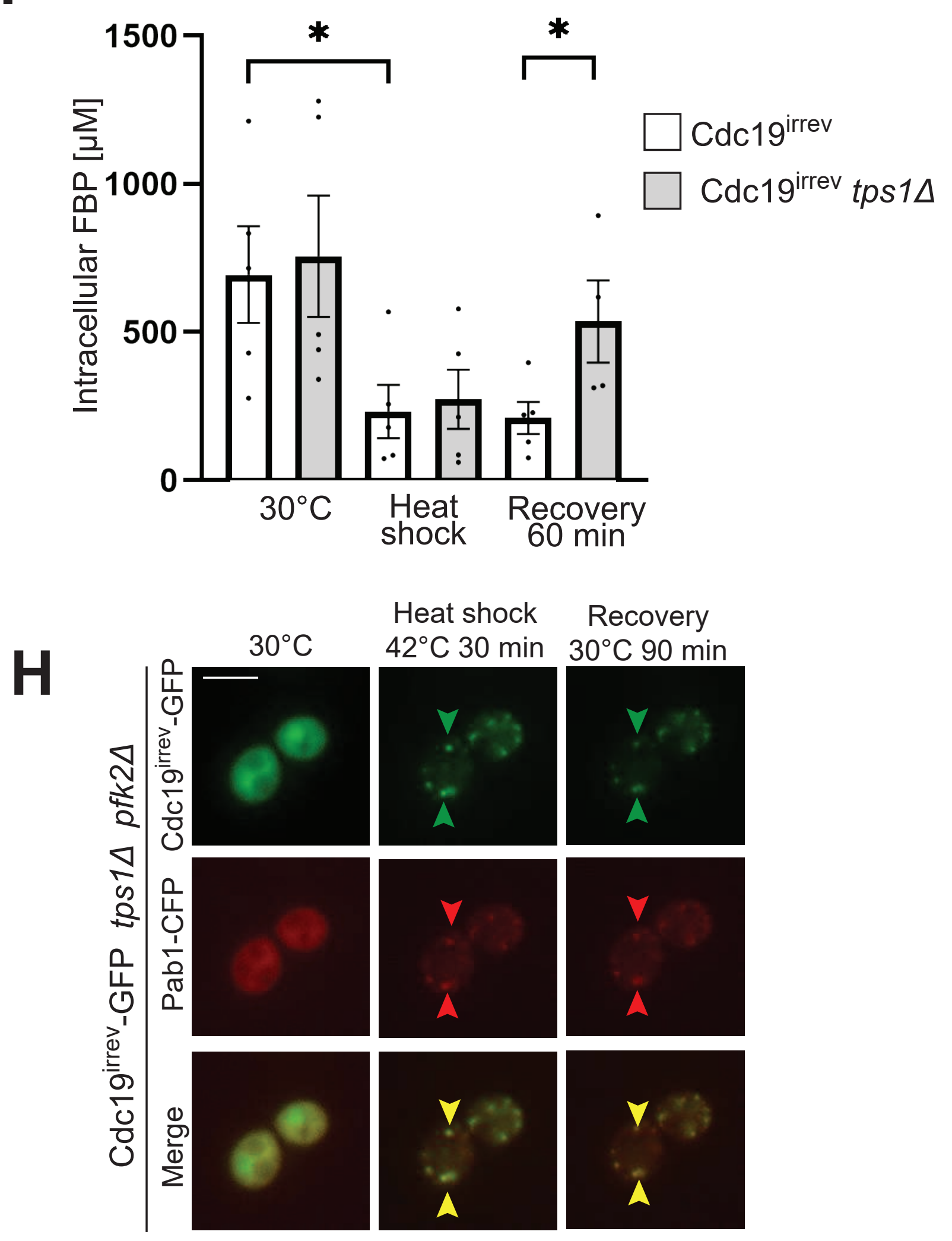


\section{by the glycolytic metabolite FBP}

(A) Schematic representation of glycolysis and trehalose metabolism. The enzymes and metabolites discussed in the following panels are highlighted. Abbreviations: glucose-6-phosphate (G6P), fructose-6-phosphate (F6P), fructose-1,6-bisphosphate (FBP), phosphoenolpyruvate (PEP), trehalose-6-phosphate (T6P), hexokinase (Hxk), trehalose synthase (Tps) and phosphofructokinase (Pfk). Note that T6P inhibits the Hxk activity (dashed blunt arrow), while FBP activates Cdc19 (dashed pointed arrow).

(B) Blocking trehalose metabolism overcomes stress-induced growth arrest of $c d c 19^{\text {irrev }}$ cells. Serial dilutions of exponentially growing wild-type, $\operatorname{tps} 1 \Delta$, $\operatorname{tps} 2 \Delta$ and $t p s 3 \Delta$ cells expressing as indicated Cdc19 $9^{\mathrm{WT}}$ or Cdc19 $9^{\text {irrev }}$. An equal number of cells was spotted on agar plates before (left) or after a 30 min heat shock at $42{ }^{\circ} \mathrm{C}$ (right), and grown at $30{ }^{\circ} \mathrm{C}$ for 3 days.

(C) Blocking trehalose metabolism allows re-solubilization of Cdc19 irrev aggregates and SG disassembly after stress. Exponentially growing $c d c 19^{\text {irrev }}$ tps $1 \Delta$ cells expressing the SG marker Pab1-CFP were grown in a microfluidic chip at $30{ }^{\circ} \mathrm{C}$ in SD-full medium (left column), heat shocked for $30 \mathrm{~min}$ at $42{ }^{\circ} \mathrm{C}$ (middle column) and allowed to recover at $30{ }^{\circ} \mathrm{C}$ (right column). Representative GFP- (top row) and CFP-images (middle row) were taken at the indicated time points, and also merged (bottom row) to show co-localization of Cdc19 aggregates and SGs (arrow heads). Data shown are representative of three independent experiments. Scale bar: 5 $\mu \mathrm{m}$.

(D) Intracellular ATP and phosphoenolpyruvate (PEP) concentrations $(\mu \mathrm{M})$ were measured in wildtype, $c d c 19^{\text {irrev }}$, and $c d c 19^{\text {irrev }} \operatorname{tps} 1 \Delta$ cells, after these cells were subjected to a 30 min heat shock at $42{ }^{\circ} \mathrm{C}$ and allowed to recover at $30{ }^{\circ} \mathrm{C}$ for $60 \mathrm{~min}$. Mean and S.E.M. determined by five independent experiments are shown (statistical analysis with Mann-Whitney test, for $c d c 19^{W T}$ - $c d c 19^{\text {irrev }}$ comparison: $P_{A T P}=0.0079$, for $c d c 19^{\text {irrev }}-c d c 19^{\text {irrev }} t p s 1 \Delta$ comparison: $P_{A T P}=$ $\left.0.0317, P_{P E P}=0.0556\right)$. Note that deletion of $t p s 1$ in $c d c 19^{\text {irrev }}$ cells allows to resume ATP production after stress release. 
(E) - (F) Intracellular disaccharide $(\mathbf{E})$ and FBP $(\mathbf{F})$ concentrations $(\mu \mathrm{M})$ were measured by massspectrometry in $c d c 19^{\text {irrev }}$ or $c d c 19^{\text {irrev }} \operatorname{tps} 1 \Delta$ strains before, during and after a 30 min heat shock at $42{ }^{\circ}$ C. Mean and S.E.M. determined by five independent experiments are shown (statistical analysis with Mann-Whitney test, for $c d c 19^{\text {irrev }} 30^{\circ} \mathrm{C}-$ Heat shock comparison: $P=0.0317$, for $c d c 19^{\text {irrev }}-c d c 19^{\text {irrev }}$ tps $1 \Delta$ Recovery 60 min comparison: $\left.P=0.0317\right)$. Note that intracellular disaccharides in yeast almost exclusively consist of trehalose [64]. Thus, in tps $1 \Delta$ cells trehalose production is impaired and FBP accumulates.

(G) Serial dilutions of exponentially growing wild-type, $p f k 2 \Delta$, tps $1 \Delta$ or tps $1 \Delta$ pfk $2 \Delta$ cells expressing either $\mathrm{Cdc} 19^{\mathrm{WT}}$ or $\mathrm{Cdc} 19^{\text {irrev }}$ were spotted on agar plates before (left) or after a 30 min heat shock of $42{ }^{\circ} \mathrm{C}$ (right). The plates were imaged after 3 days at $30{ }^{\circ} \mathrm{C}$.

(H) Exponentially growing tps $1 \Delta p f k 2 \Delta$ cells expressing the SG marker Pab1-CFP and the GFPtagged Cdc19 $9^{\text {irrev }}$ mutant were grown in a microfluidic chip at $30{ }^{\circ} \mathrm{C}$ in SD-full medium (left column), subjected to heat stress for $30 \mathrm{~min}$ at $42{ }^{\circ} \mathrm{C}$ (middle column) and allowed to recover at $30{ }^{\circ} \mathrm{C}$ (right column). Representative GFP- (top row) and CFP-images (middle row) were taken at the indicated time points, and also merged (bottom row) to show co-localization of Cdc19 aggregates and SGs (arrow heads). The intensity of the GFP and CFP images before heat shock has been slightly reduced to allow better visualization. Scale bar: $5 \mu \mathrm{m}$. Note that deleting the FBP-producing enzyme Pfk2 not only abolishes growth restart observed in tps $1 \Delta$ cells, but also Cdc19 $9^{\text {irrev }}$ aggregate re-solubilization and SG disassembly. 
Figure 3 - FBP directly regulates formation and disassembly of reversible Cdc19 amyloids

A
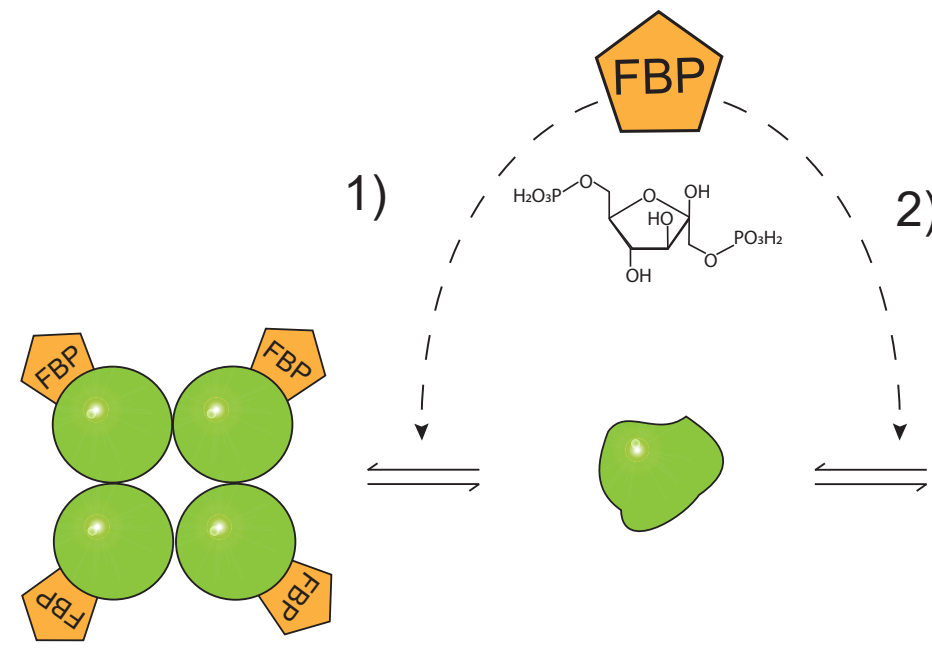

Soluble Cdc19

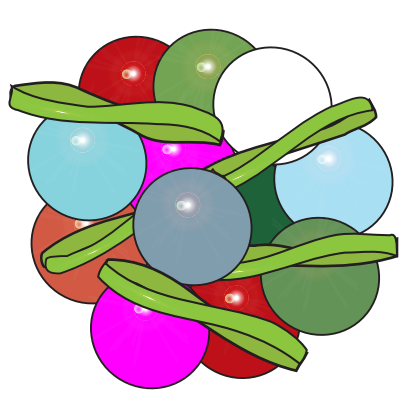

Stress granule (Cdc19 amyloids)
B
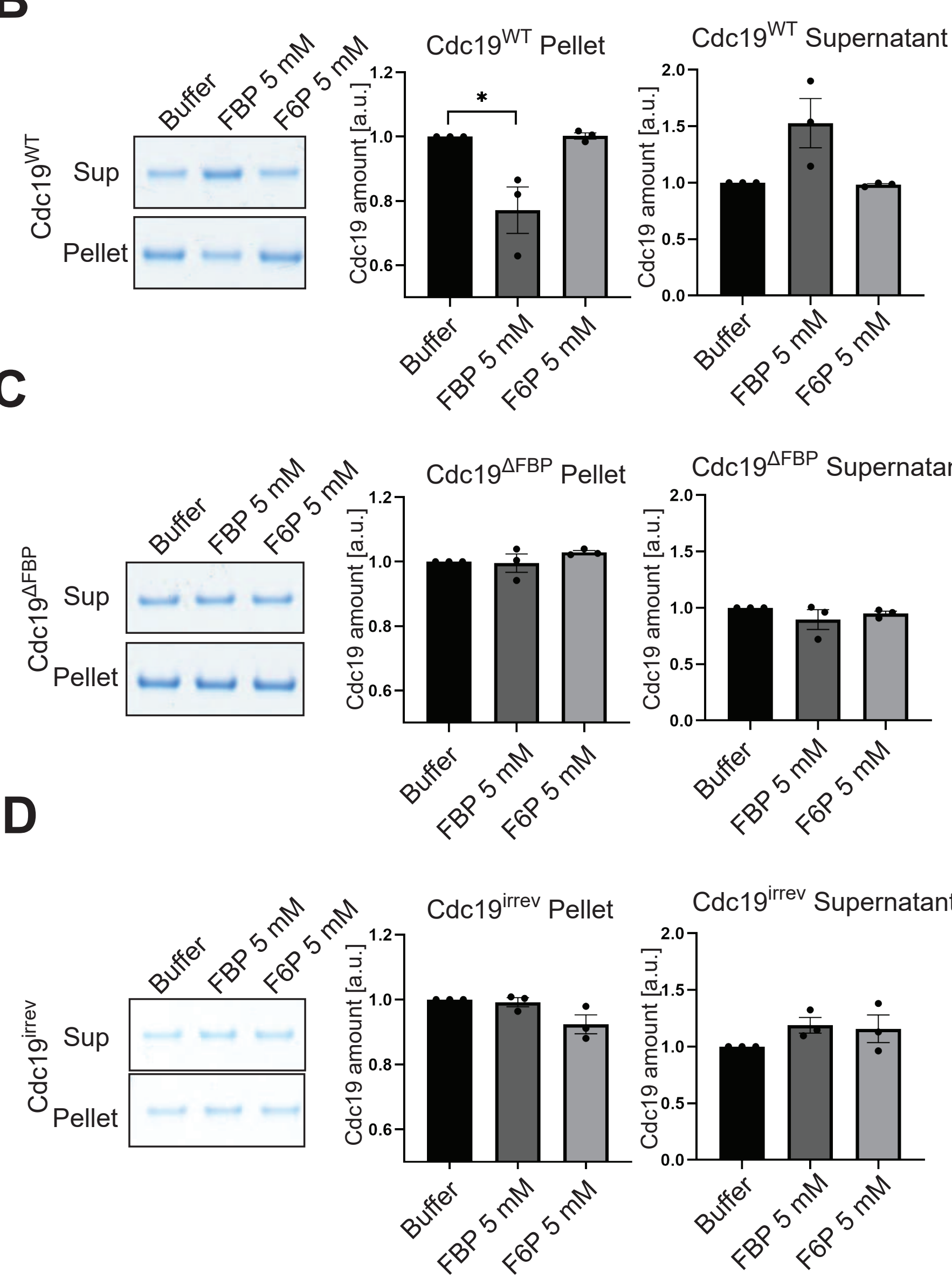

E

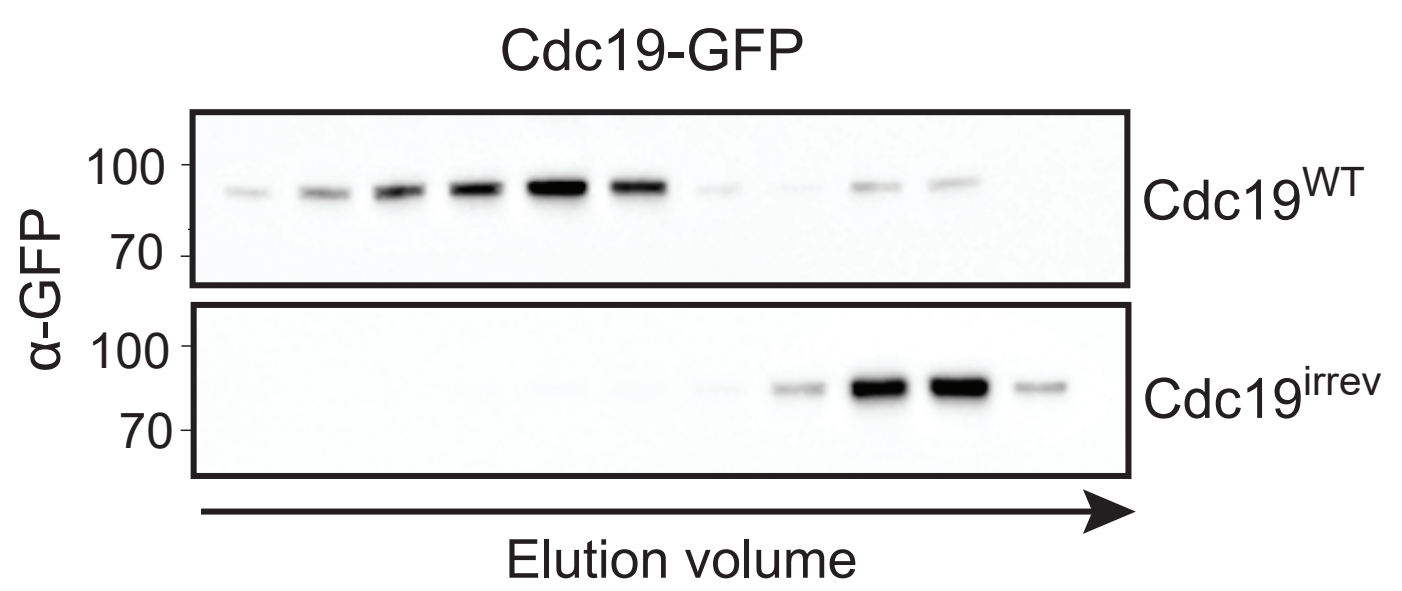

F

SEC with purified Cdc19*

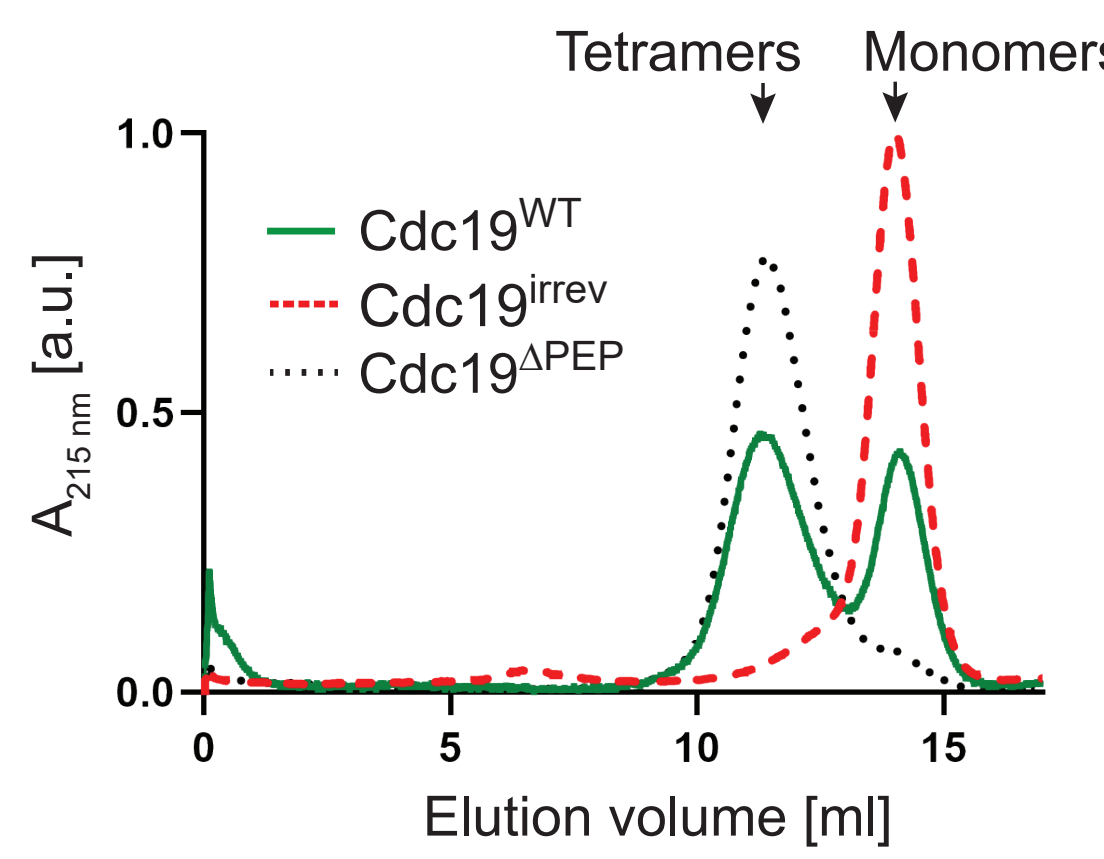

G

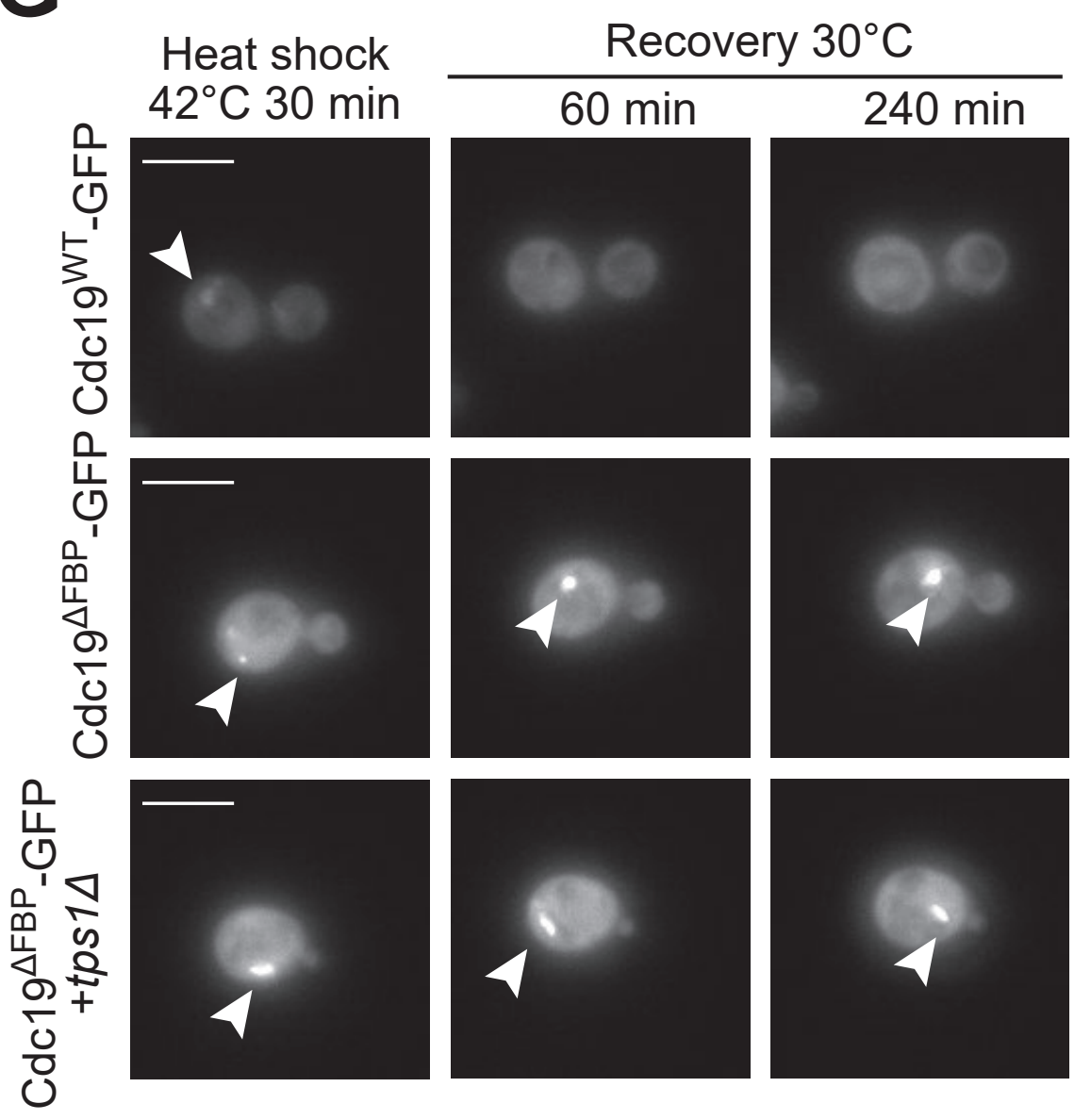

H
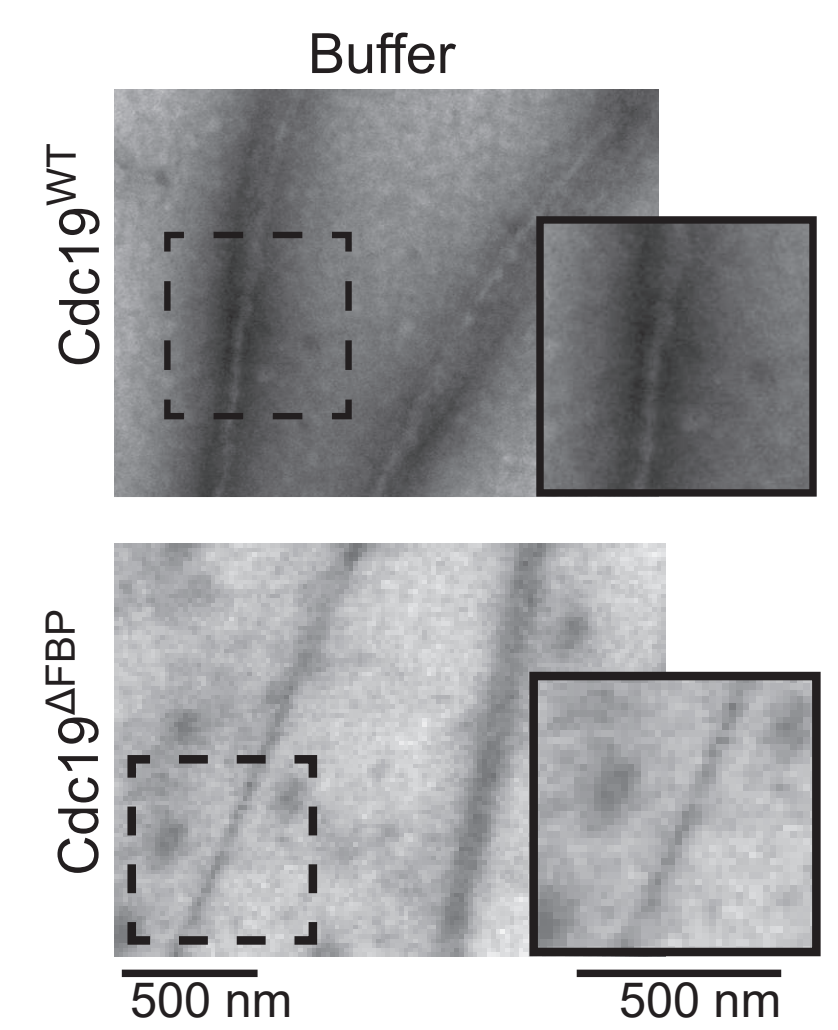

+ FBP
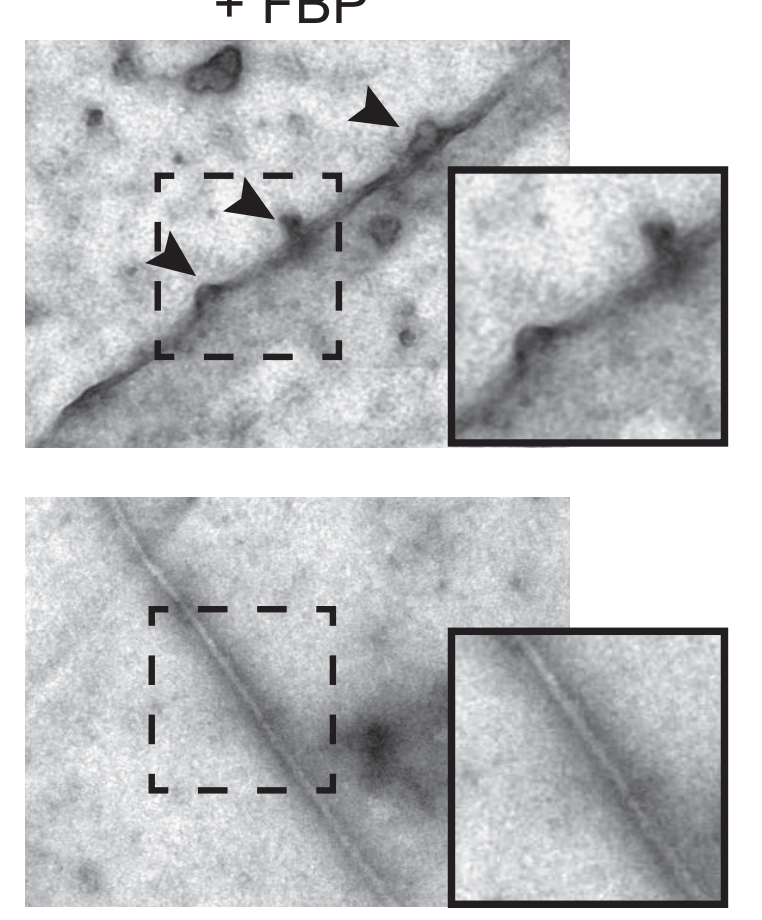

$+\mathrm{F} 6 \mathrm{P}$

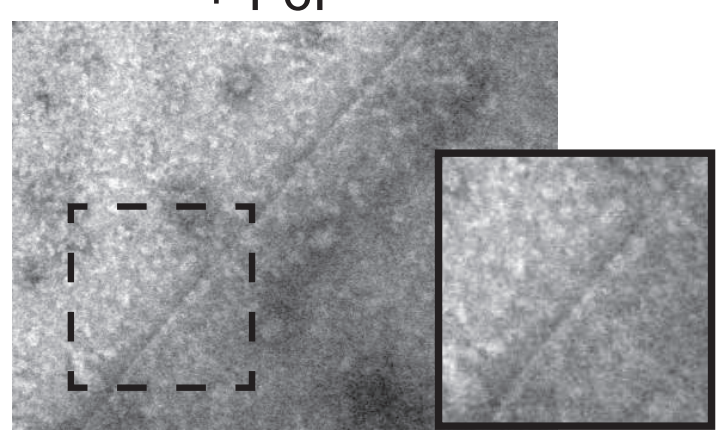


(A) Schematic representation of how FBP may regulate Cdc19 amyloid formation and disassembly. FBP binds soluble Cdc19 and promotes its tetramerization. Since Cdc19 tetramers are less aggregation-prone than the monomers [4], FBP may help to maintain a soluble Cdc19 pool, thus reducing aggregate formation (dashed arrow 1). Alternatively, FBP may also bind Cdc19 amyloids and promote aggregate re-solubilization (dashed arrow 2). Note that the two regulation.

(B) - (D) Binding of FBP to soluble Cdc19 ${ }^{\mathrm{WT}}$ reduces its aggregation in vitro. Purified wild-type Cdc19 (B), FBP binding-deficient Cdc19 ${ }^{\triangle \mathrm{FBP}}$ mutant (C) or Cdc19 $9^{\text {irrev }}$ mutant (D) proteins were mixed with $5 \mathrm{mM}$ FBP or for control $5 \mathrm{mM} \mathrm{F6P}$ or buffer and incubated at $30^{\circ} \mathrm{C}$ for 14 hours. Cdc19 aggregates were separated from soluble Cdc19 by centrifugation and the supernatant (Sup, containing soluble Cdc19) and pellet (Pellet, containing aggregated Cdc19) fractions were analysed by SDS-PAGE and Coomassie blue staining (left gels). Cdc19 amount was quantified in the Pellet (left graphs) and Supernatant (right graphs) by measuring Cdc19 band intensity using ImageJ and normalizing it to buffer control, and is shown as graphs with mean \pm S.E.M. from three independent experiments (statistical analysis with two-tailed t-test, $P=$ $0.0342)$.

(E) - (F) $\mathrm{Cdc} 19^{\text {irrev }}$ is exclusively monomeric in vivo and in vitro. (E) Extracts from cells expressing GFP-tagged Cdc19 ${ }^{\text {WT }}$ (top panel) or Cdc19 $9^{\text {irrev }}$ (bottom panel) were separated by size-exclusion chromatography (SEC) and selected peak fractions were analysed by Western blot with an $\alpha$ GFP antibody. The elution volume (ml) is indicated below, and the numbers on the left indicate size markers (in $\mathrm{kDa})$. (F) Recombinant wild-type $\left(\mathrm{Cdc} 19^{\mathrm{WT}}\right), \mathrm{Cdc} 19^{\text {irrev }}$ or for control tetrameric Cdc $19^{\triangle \mathrm{PEP}}$ mutant proteins were purified and analysed by SEC. Absorbance at $215 \mathrm{~nm}$ (arbitrary units) was normalized and plotted as a function of elution volume (ml). Data are representative of three independent experiments. 
(G) Direct FBP binding to Cdc19 is necessary to re-solubilize Cdc19 amyloids in vivo. Wild-type or tps $1 \Delta$ cells expressing GFP-tagged wild-type Cdc19 (top row) or the FBP binding-defective Cdc19 ${ }^{\triangle \mathrm{FBP}}$ mutant (middle and bottom row) and overexpressing Pyk2 were grown in a microfluidic chip at $30{ }^{\circ} \mathrm{C}$ in SD-full medium, subjected to heat stress for $30 \mathrm{~min}$ at $42{ }^{\circ} \mathrm{C}$ (left column) and allowed to recover at $30{ }^{\circ} \mathrm{C}$ in the presence of $25 \mu \mathrm{g} / \mathrm{ml}$ cycloheximide to avoid $d e$ novo protein synthesis. Representative GFP-images were taken 60 (middle column) or $240 \mathrm{~min}$ (right column) after heat shock end. Cdc19-GFP aggregates are marked with arrowheads. Scale bars: $5 \mu \mathrm{m}$.

(H) Binding of FBP to pre-formed $\mathrm{Cdc} 19^{\mathrm{WT}}$ amyloids triggers conformational changes visible by negative staining transmission electron microscopy (TEM) using uranyl acetate stain. Purified Cdc19 ${ }^{\text {WT }}$ (upper row) or the FBP binding-defective Cdc19 ${ }^{\triangle \mathrm{FBP}}$ mutant were aggregated by a 10 min heat shock at $42{ }^{\circ} \mathrm{C}$, and the resulting amyloids were incubated with FBP (4 mM) or for control F6P (4 mM) or buffer. Scale bar: $500 \mathrm{~nm}$. Arrows mark FBP-induced "bulges" that are frequently observed along the fibrils axis. The indicated sections (dashed squares) of representative fibrils were enlarged 1.5 times to better visualize the distinct morphologies (inserts). Images are representative of three independent experiments. 
Figure 4 - FBP binding to Cdc19 amyloids promotes chaperone recruitment triggering Cdc19 re-solubilization and SG disassembly in vivo

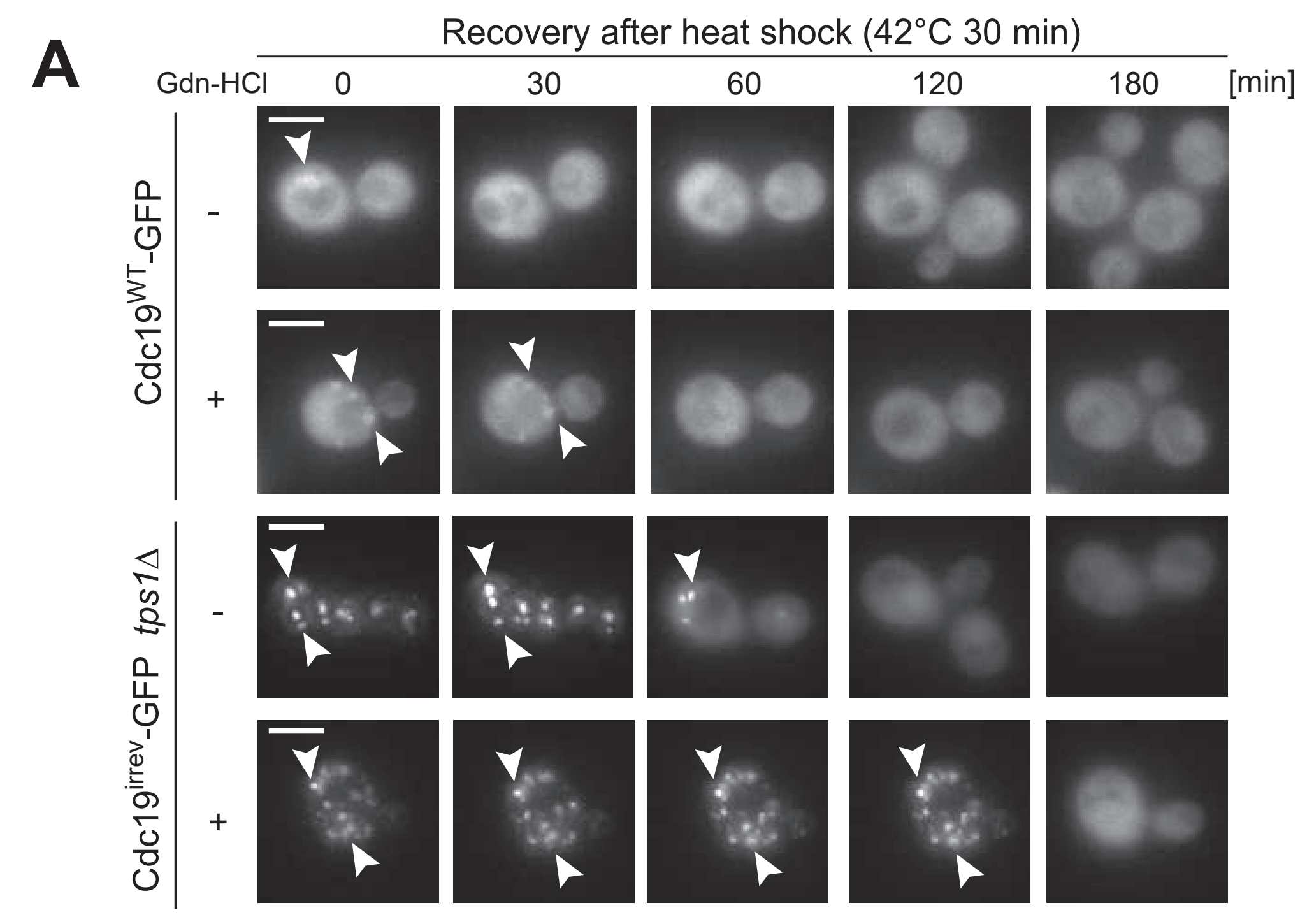

B

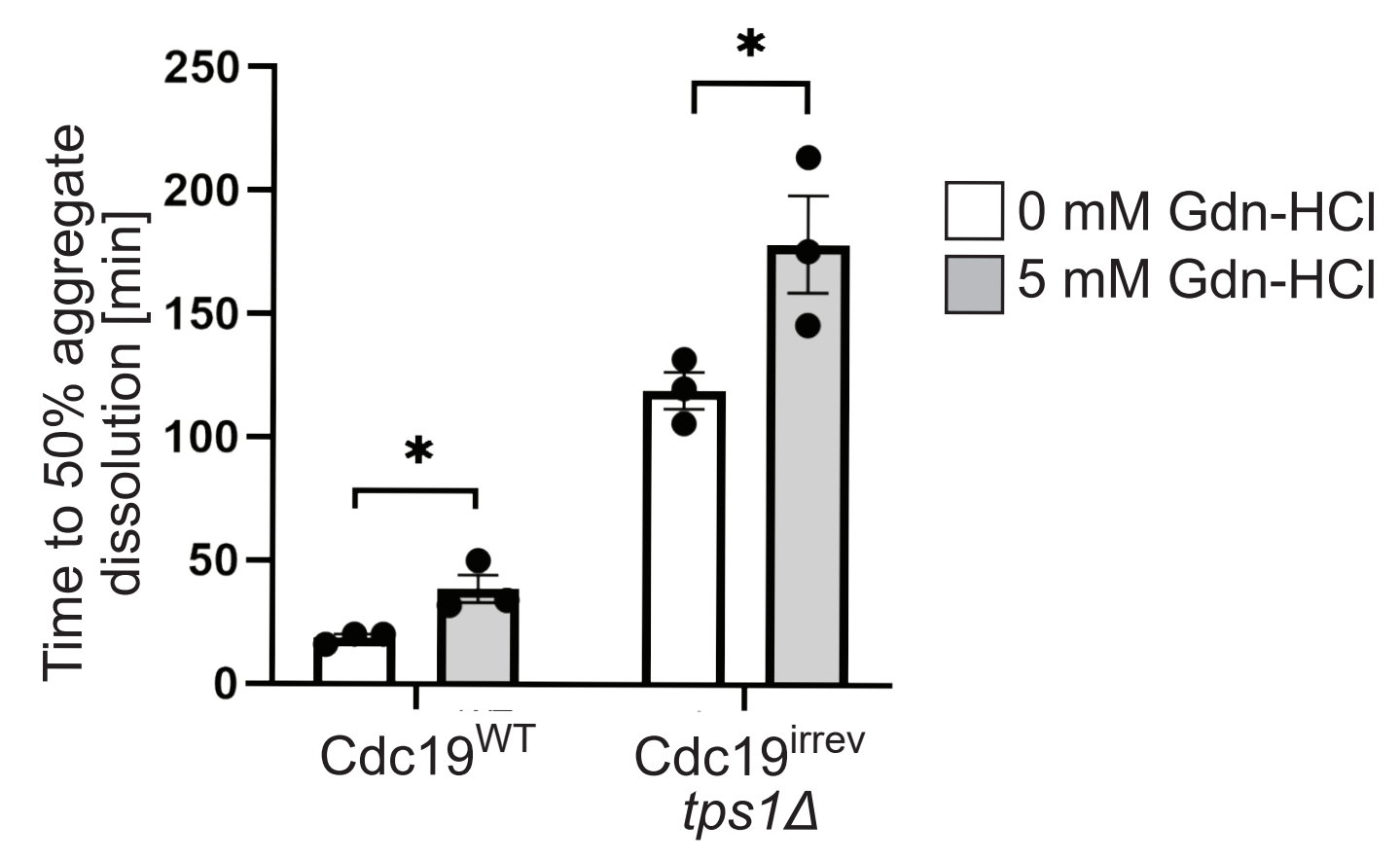

C

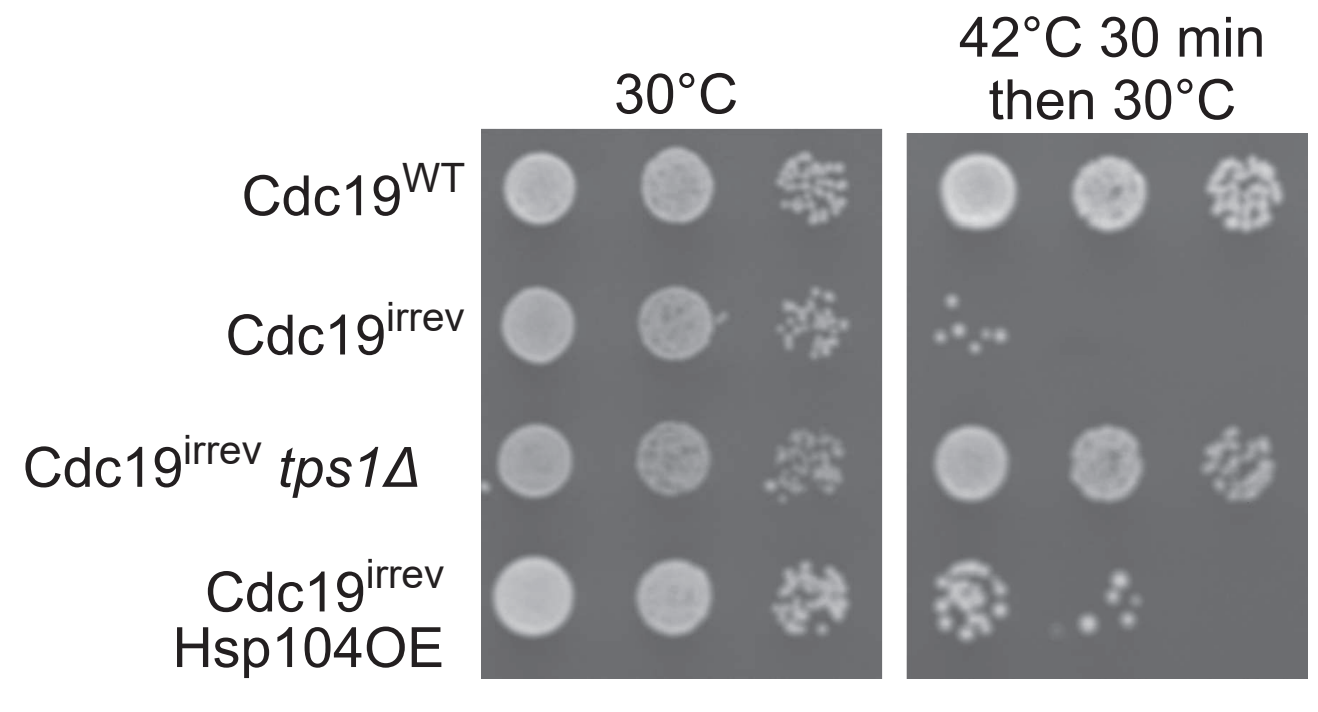

E
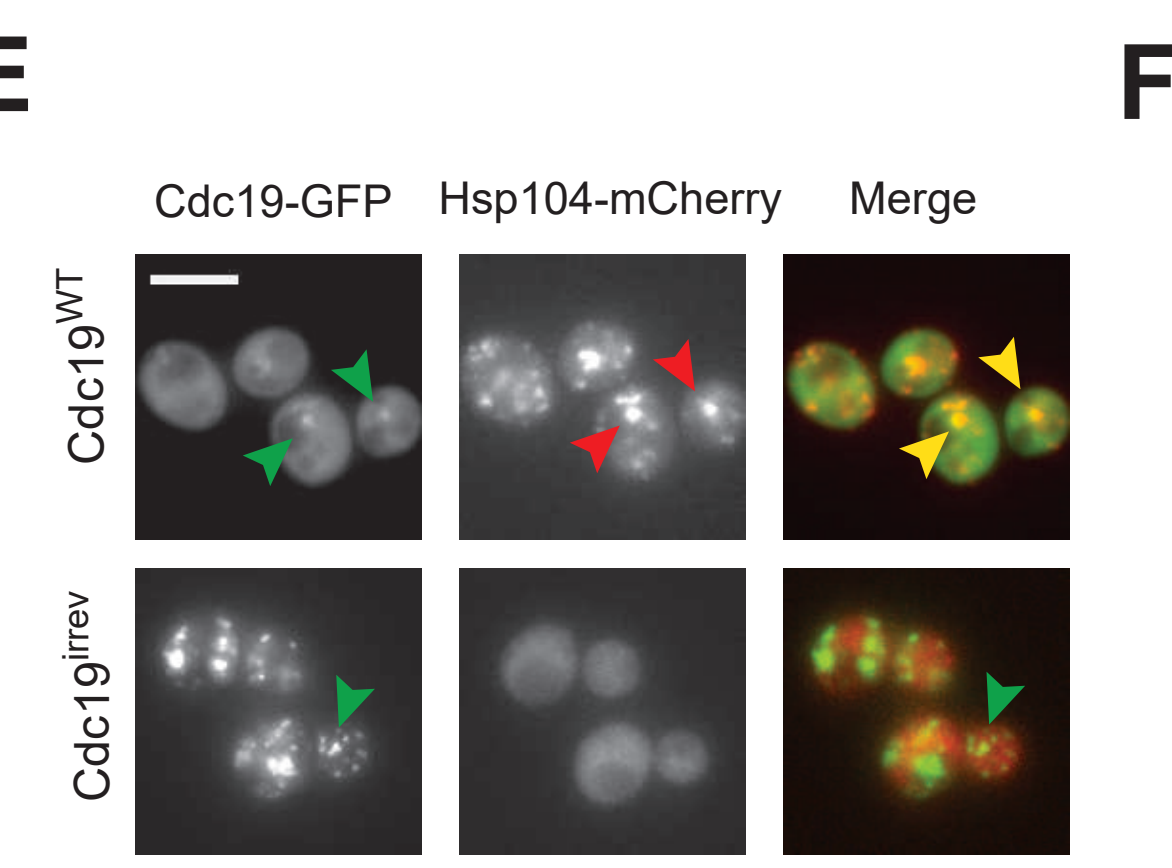

F
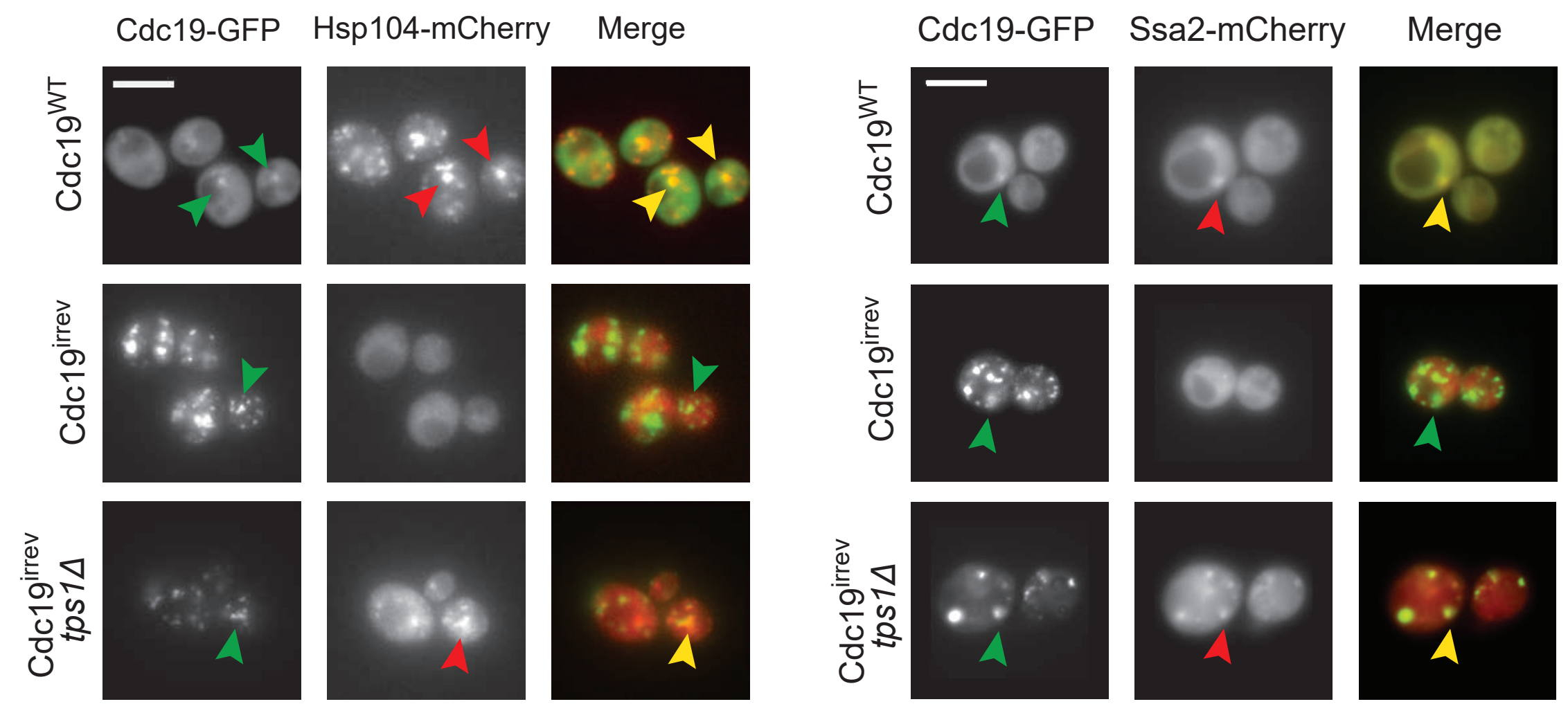

D

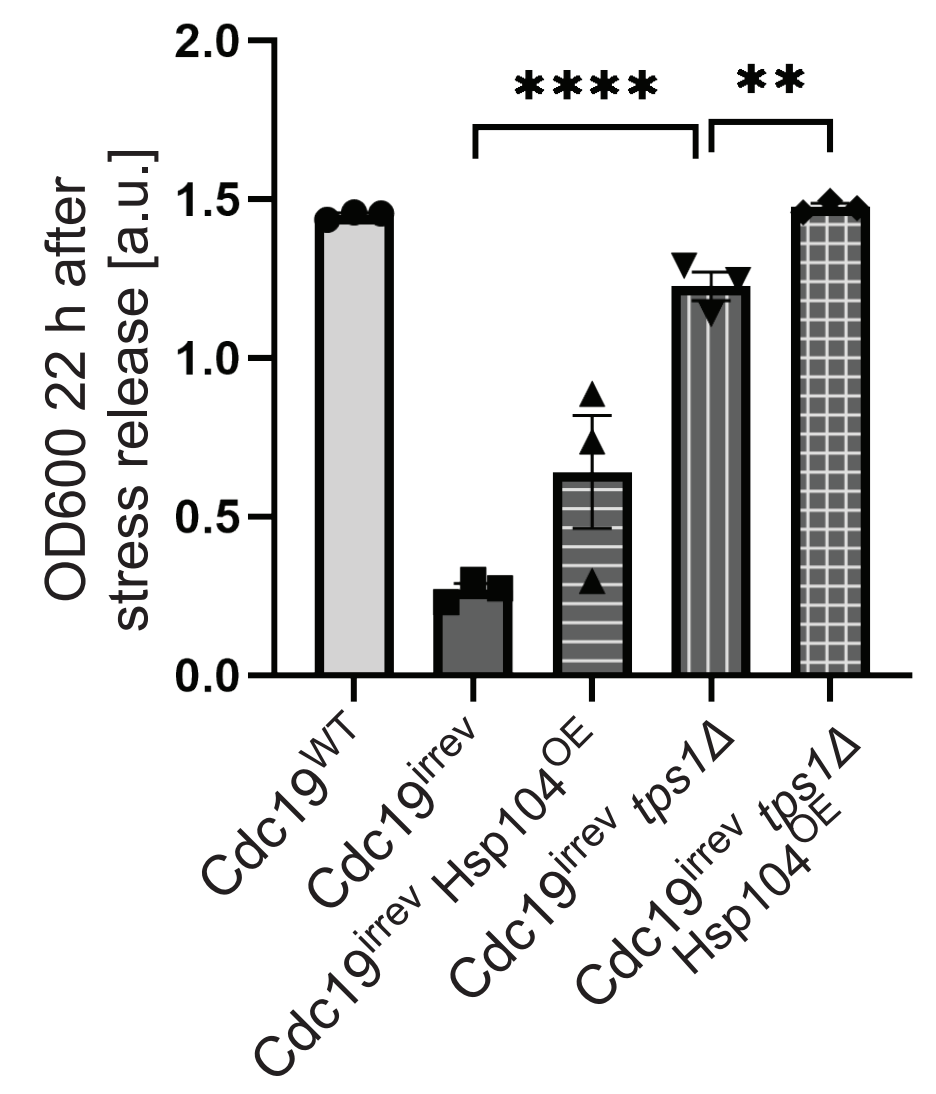

G
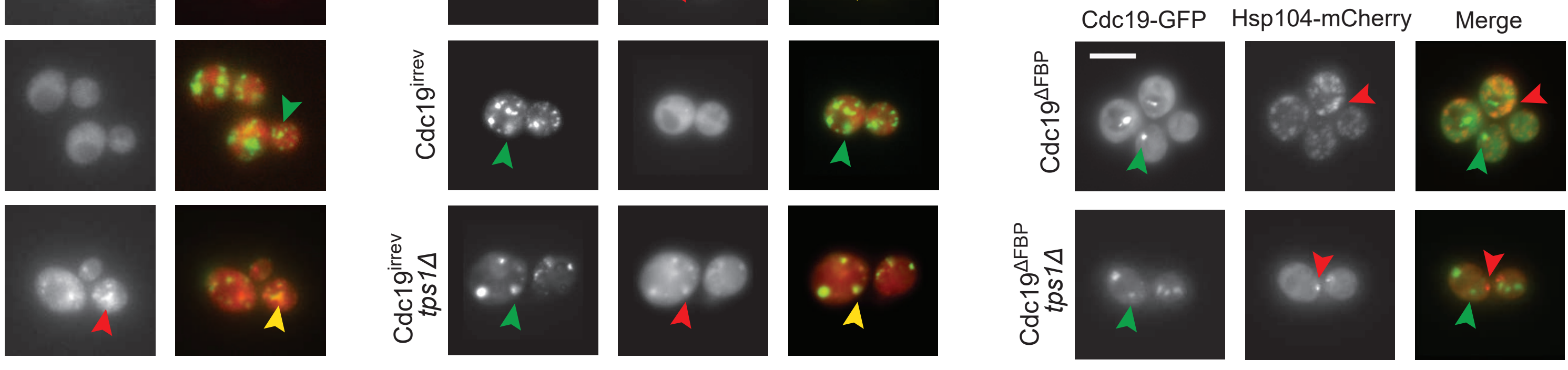

Cdc19*-GFP and Hsp104-mCherry

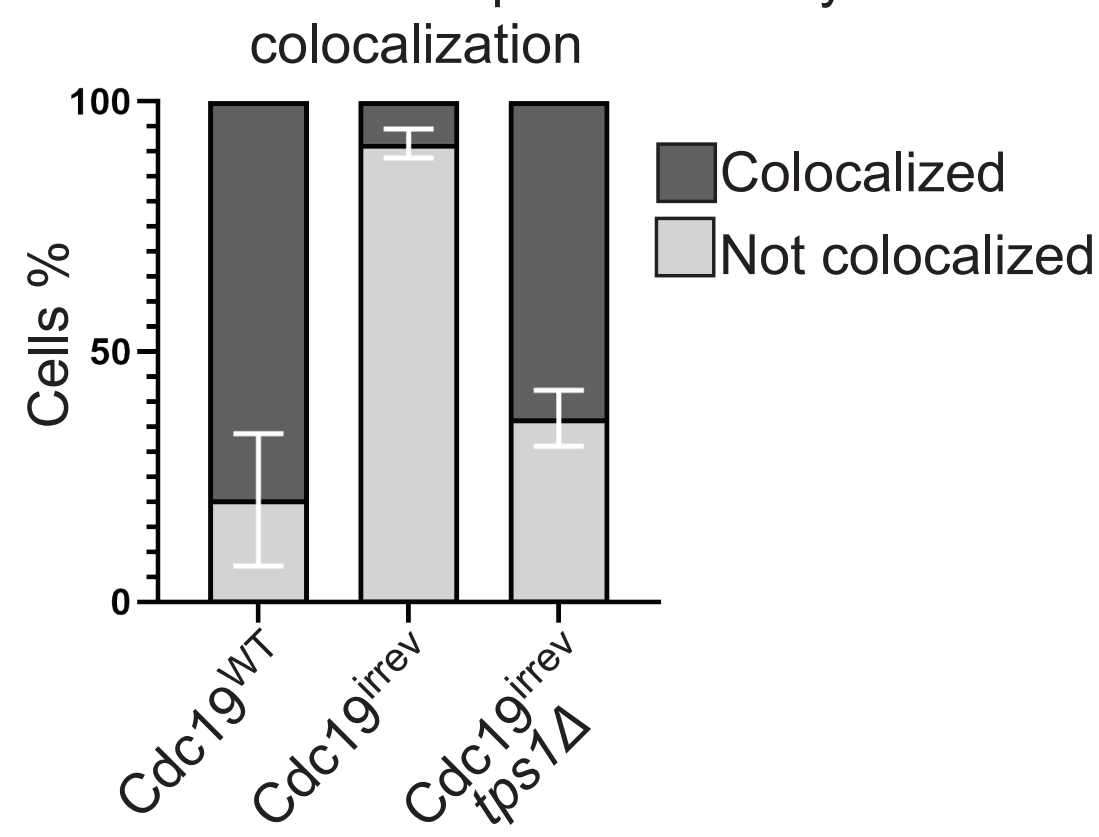

Cdc19*-GFP and Ssa2-mCherry colocalization

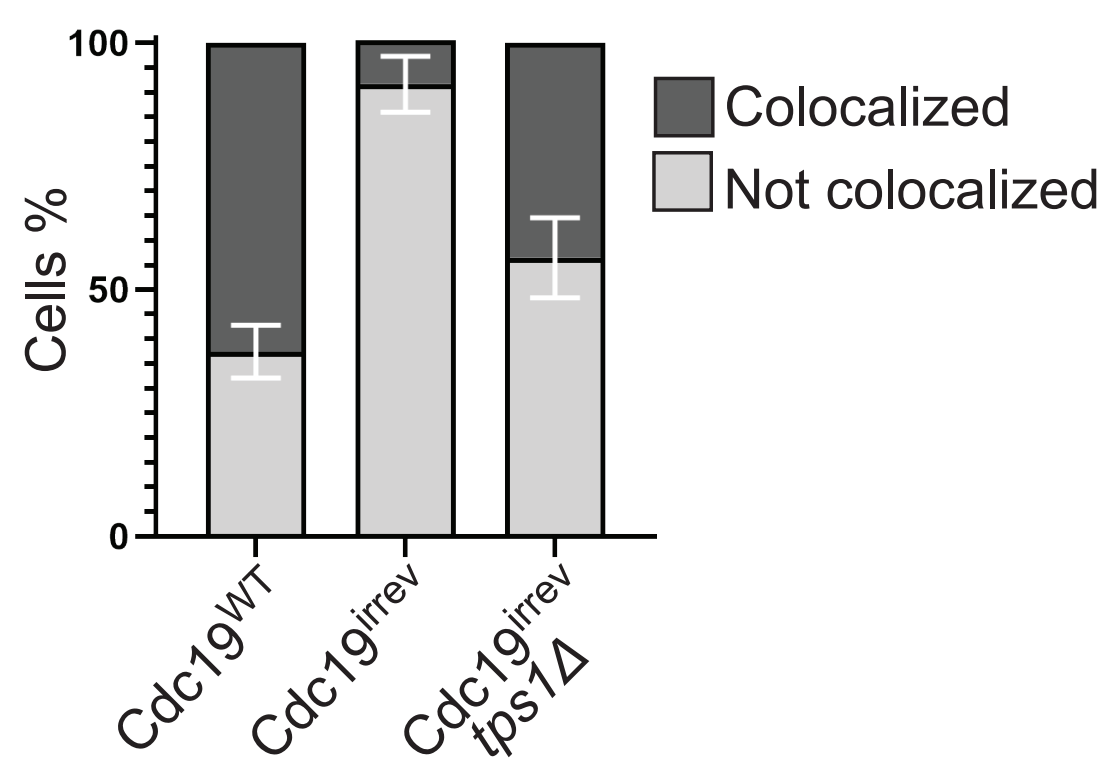

Cdc19*-GFP and Hsp104-mCherry colocalization

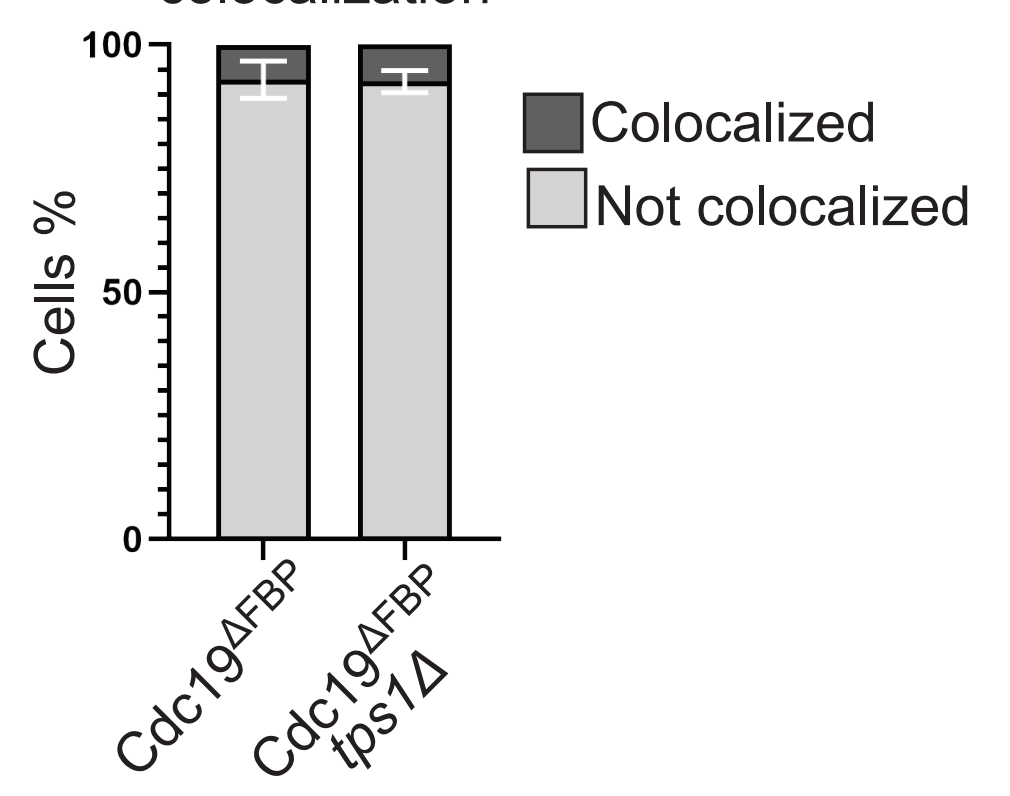


(A) - (B) Inhibition of Hsp104 activity delays re-solubilization of Cdc19 ${ }^{\text {WT }}$ and Cdc19 irrev aggregates in wild-type and tps $1 \Delta$ cells, respectively. Wild-type and tps $1 \Delta$ cells expressing respectively GFP-tagged wild-type Cdc19 or the $\mathrm{Cdc} 19^{\text {irrev }}$ mutant were treated with $5 \mathrm{mM}$ of the $\mathrm{Hsp} 104$ inhibitor $\mathrm{Gdn}-\mathrm{HCl}$ for 3 hours prior to a 30 min heat shock at $42{ }^{\circ} \mathrm{C}$. (A) Aggregate disassembly after stress release was monitored at the indicated time points by fluorescence microscopy. Representative images of three independent experiments are shown, with arrowheads marking Cdc19 aggregates. Scale bar: $5 \mu \mathrm{m}$. (B) Cdc19 aggregate disassembly was quantified by counting the number of foci at different time points after stress. The average time to reach a $50 \%$ reduction in foci number is displayed. Error bars represent S.E.M. (statistical

C) - (D) Overexpression of Hsp104 partially rescues growth of $c d c 19^{\text {irrev }}$ cells after heat shock. (C) Serial dilutions of exponentially growing wild-type or tps1 $\Delta$ cells expressing either $\mathrm{Cdc} 19^{\mathrm{WT}}$ or $\mathrm{Cdc} 19^{\text {irrev }}$ were spotted on agar plates before (left) or after a 30 min heat shock of $42{ }^{\circ} \mathrm{C}$ (right). Where indicated, overexpression of Hsp104 was induced by treating cells with $10 \mathrm{mM}$ estradiol for 3 hours. The plates were imaged after 3 days at $30^{\circ} \mathrm{C}$. (D) Growth restart of the indicated strains treated as described above was also quantified by measuring the cell density $\left(\mathrm{OD}_{600}\right)$ over time after inoculation of equal cell numbers at $30{ }^{\circ} \mathrm{C}$. Mean cell density 22 hours after stress release is shown, with error bars representing S.E.M. from three independent experiments (statistical analysis with two-tailed t-test, $P_{\text {irrev-irrev }+ \text { tps } I \Delta}<0.0001$, $\left.P_{\text {irrev }+ \text { tps } 14-\text { irrev }+ \text { tps } 14+H s p 104 O E}=0.0055\right)$. Note that Hsp104 overexpression and increased FBP levels co-operate to rescue $c d c 19^{\text {irrev }}$ cells after heat shock.

(E) - (G) FBP binding to Cdc19 amyloids promotes chaperone recruitment, which correlates with aggregate disassembly. Wild-type or tps $1 \Delta$ cells co-expressing mCherry-tagged Hsp104 (E, G) or Ssa2 (F) and either GFP-tagged Cdc19 ${ }^{\mathrm{WT}}$, Cdc19 $9^{\text {irrev }}$ or $\mathrm{Cdc} 19^{\triangle \mathrm{FBP}}$ were subjected to heat stress for $30 \mathrm{~min}$ at $42{ }^{\circ} \mathrm{C}$ and imaged by fluorescence microscopy. The $c d c 19^{\triangle F B P}$ cells (G) 
overexpress Pyk2 to restore ATP production and cell viability. Representative GFP- (left column) and mCherry-images (middle column) are shown, together with the merged image (right column) to visualize co-localization of Cdc19 aggregates and these chaperones. Images are representative of three independent experiments. Scale bar: $5 \mu \mathrm{m}$. The percentage (\%) of cells where Cdc19 and Hsp104 or Ssa2 co-localized was quantified and plotted as mean with S.E.M. in the bar graph. At least 300 cells were analysed for each condition. 
Figure 5 - Molecular network coupling cellular metabolism with reversible Cdc19 amyloids and stress granule dynamics

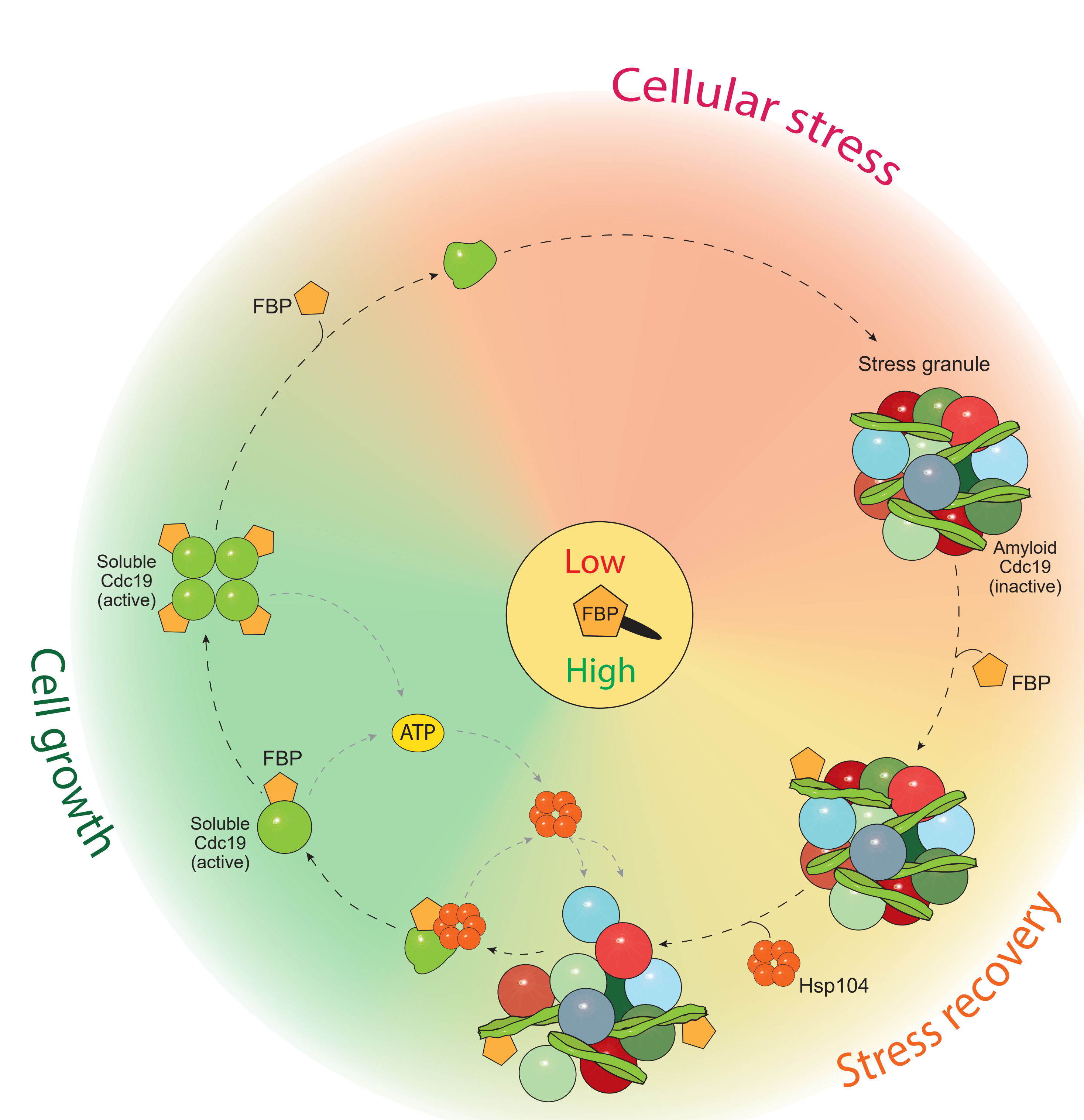


The metabolite FBP couples cellular metabolism to the assembly state and activity of Cdc19, which in turn regulates SG dynamics. During cell growth, FBP is produced through glycolysis tetramers. Upon stress, FBP levels rapidly drop, leading to tetramer disassembly and the accumulation of Cdc19 monomers. In this state, Cdc19 exposes its aggregation-prone LCR and is primed to form Cdc19 amyloids, which are inactive and accumulate in SGs. After stress release, FBP levels rise and FBP directly binds to Cdc19 amyloids, thereby triggering a conformational change along the Cdc19 fibrils that facilitates the recruitment of Hsp104 and Ssa2. These chaperones allow the re-solubilisation of Cdc19 amyloids and the release of Cdc19 monomers. Re-solubilised, active Cdc19 in turn uses PEP to produce ATP, which is required to activate chaperones to further solubilize $\mathrm{Cdc} 19$ and increase energy production. This positive feedback loop fuels disassembly of Cdc19 amyloids and other SG components. Concomitant breakdown of trehalose releases glucose moieties for glycolysis and modulates cytoplasmic diffusion. Orchestrated disassembly of SGs then releases several mRNAs and metabolic enzymes, and allows full resumption of cell growth and cell cycle progression. 

A
B

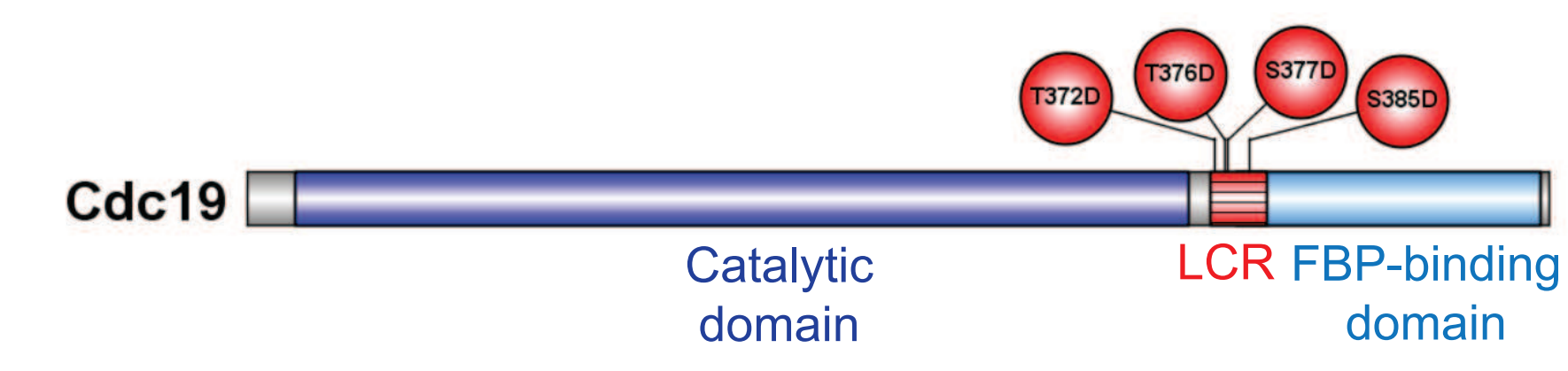

D
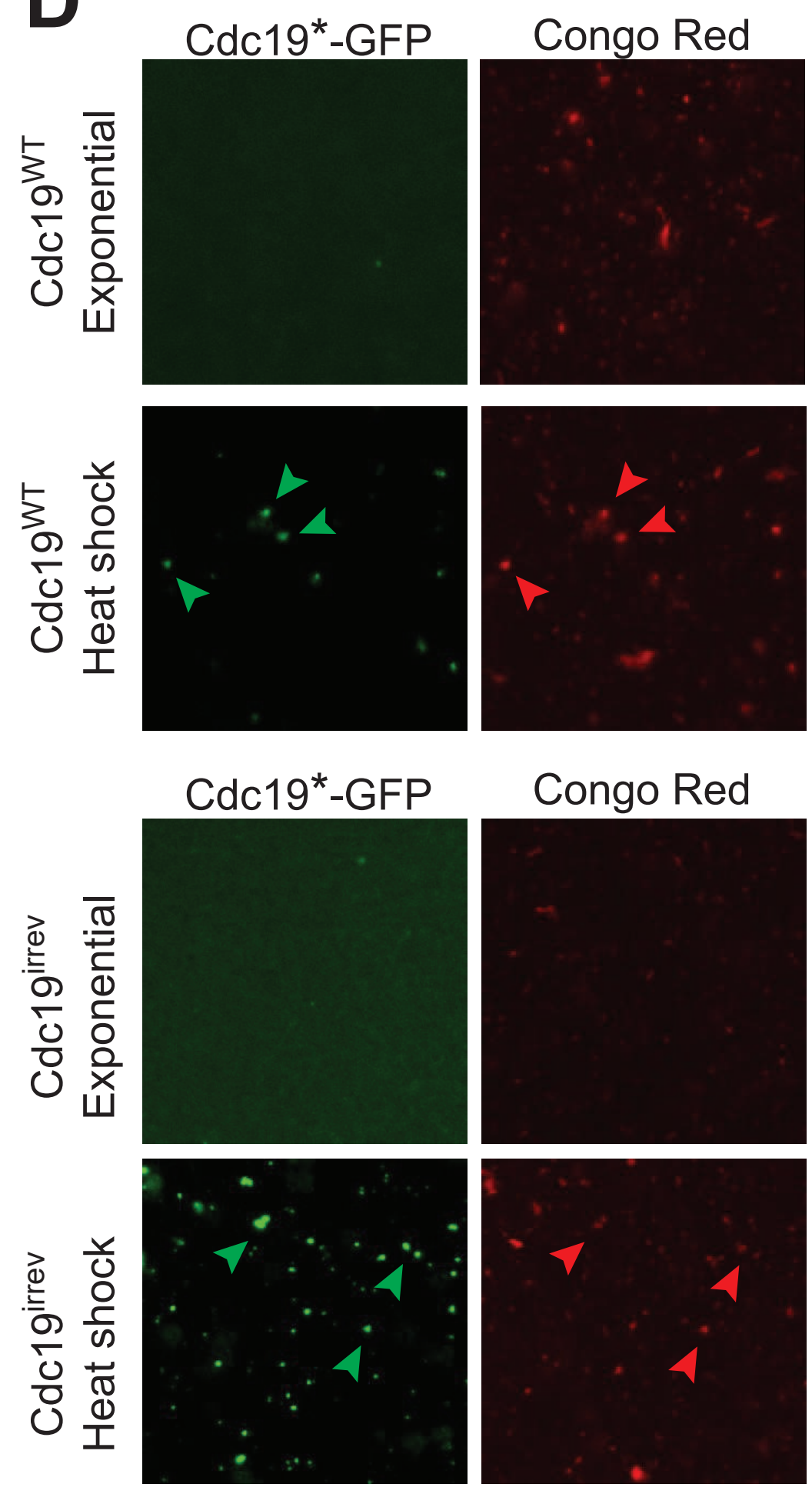

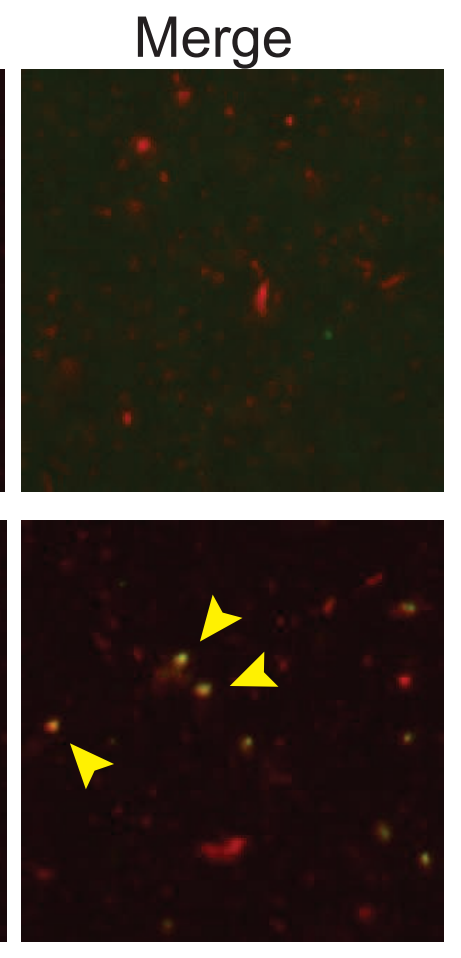

ThT emission at $490 \mathrm{~nm}$

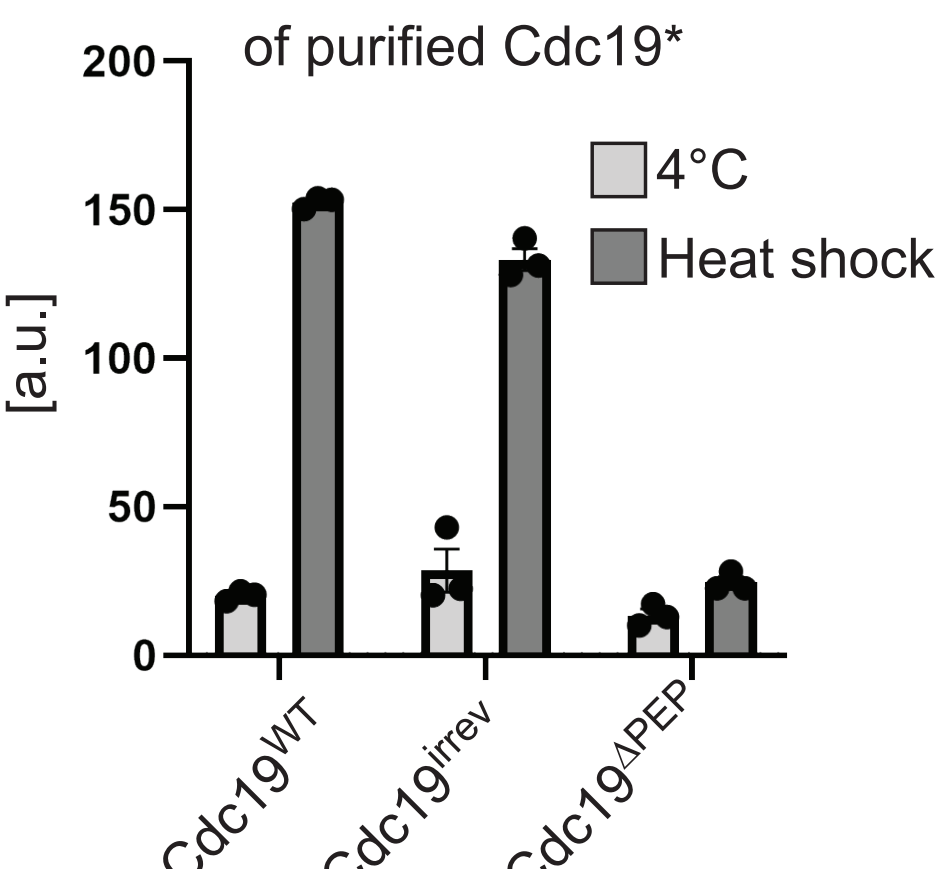

E
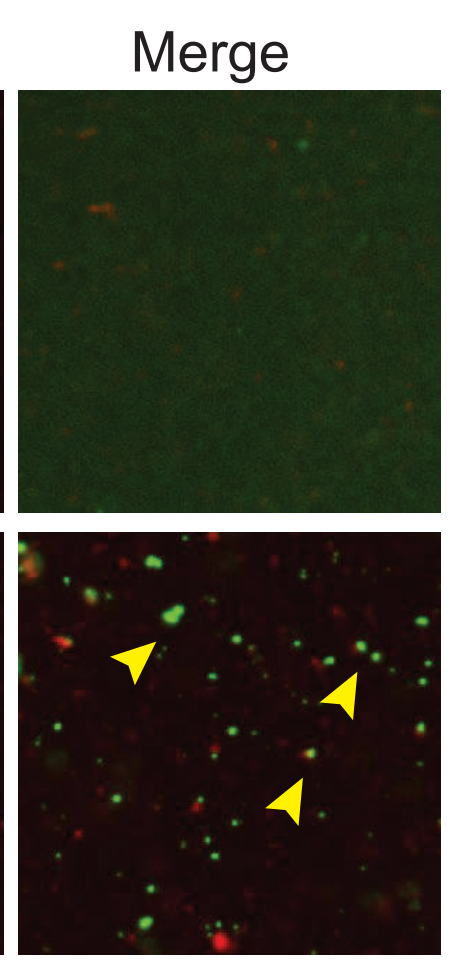

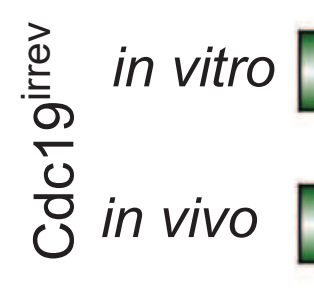

C

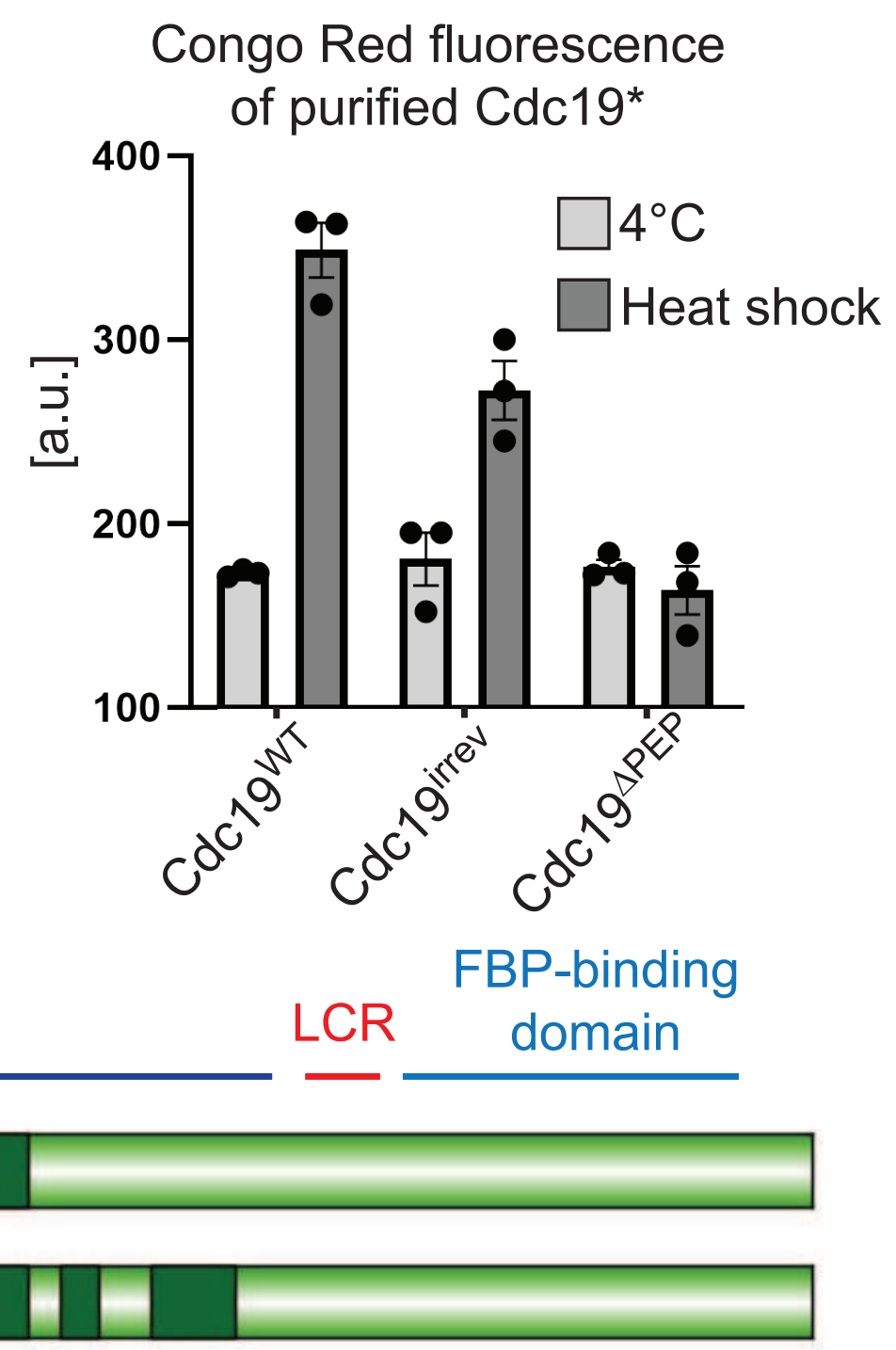

Regions undergoing structural changes upon aggregation

Changes in Cdc19 ${ }^{\text {WT }}$ upon heat shock Changes in Cdc19irrev upon heat shock

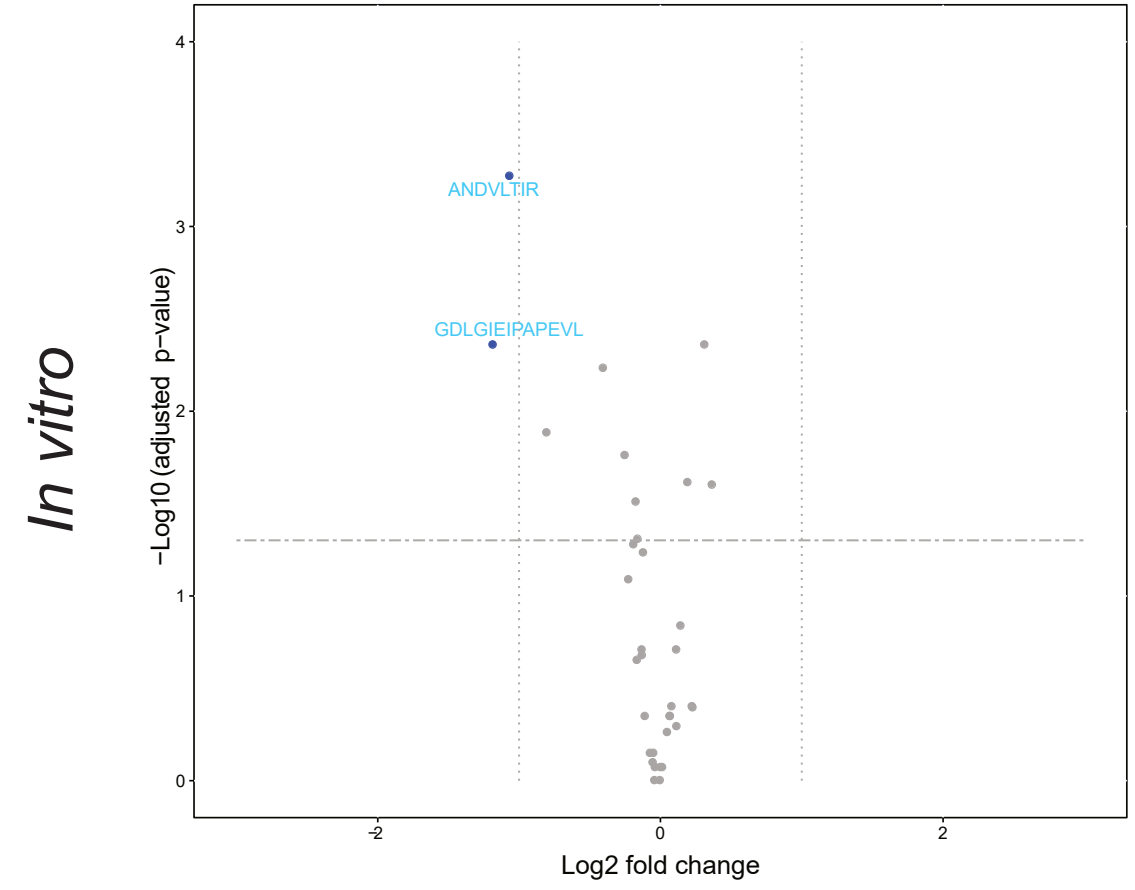

Changes in Cdc19wT upon starvation

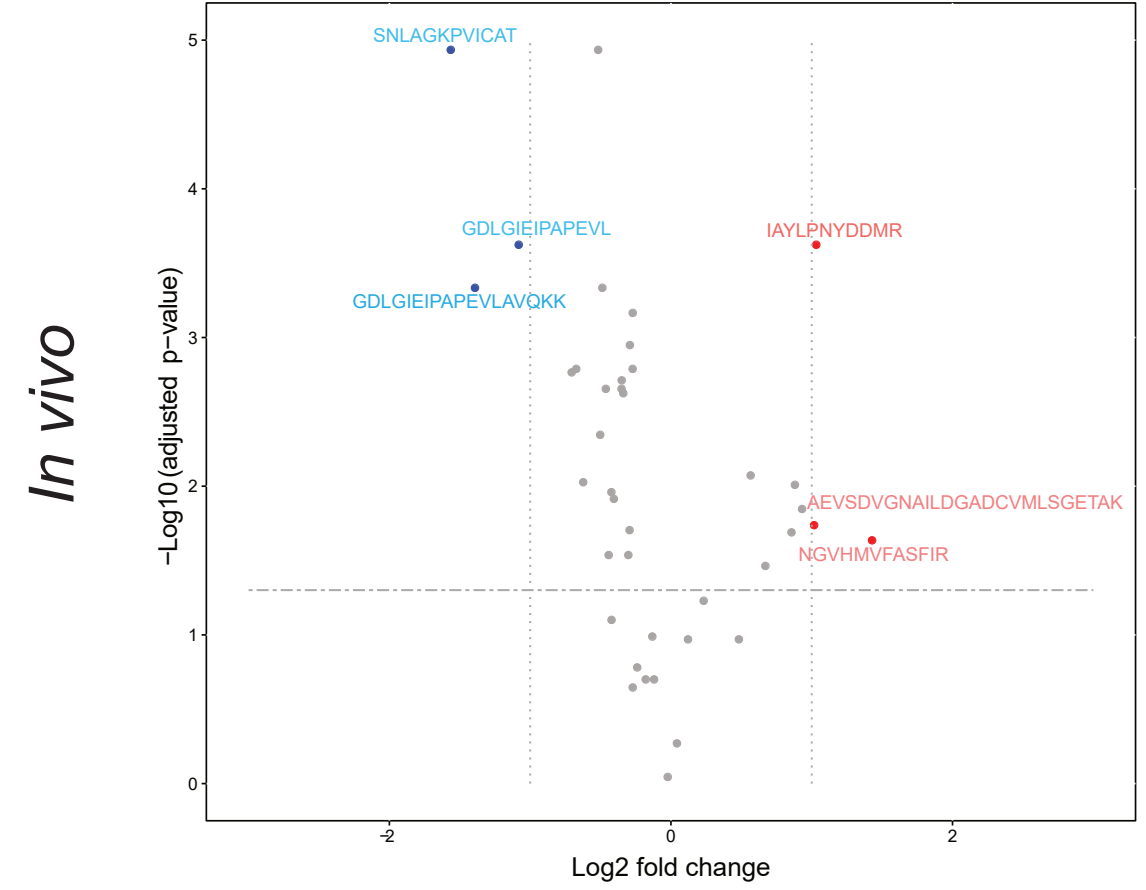

- Not regulated Adjusted p-value cutoff

- Down-regulated Fold change cutoff

- Up-regulated
Changes in Cdc19irrev upon starvation
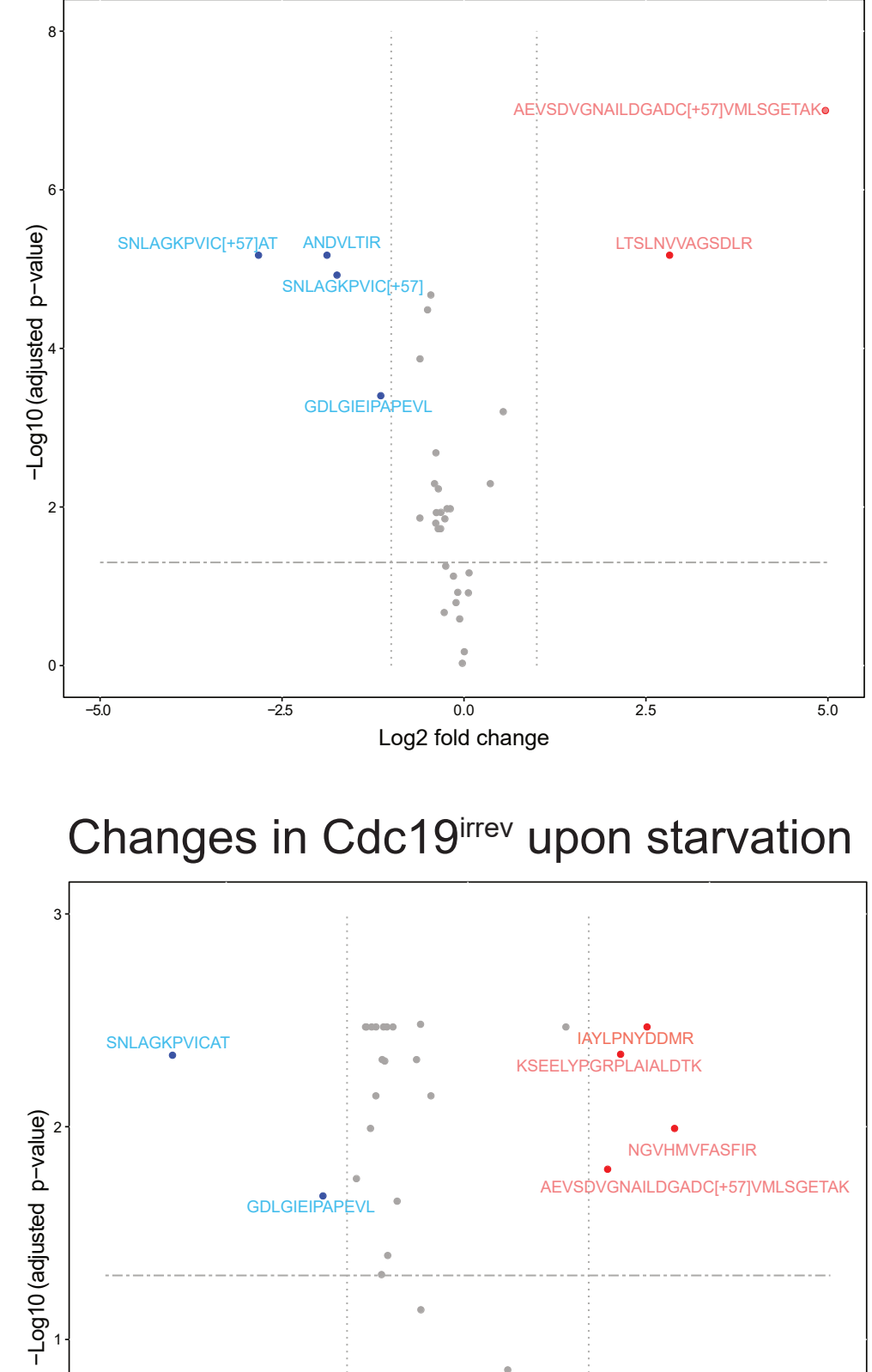

Loge fold change 


\section{Suppl. Figure 1: Cdc19 aggregates are amyloids both in vitro and in vivo}

(A) Schematic representation of the domains of Cdc19. The catalytic domain, FBP-binding domain, and the aggregation-prone low complexity region (LCR) are highlighted. The four mutated residues located within the LCR in the Cdc19 $9^{\text {irrev }}$ mutant are indicated (red circles).

(B) - (C) Both Cdc19 ${ }^{\mathrm{WT}}$ and Cdc19 $9^{\text {irrev }}$ form ThT- and Congo Red-positive aggregates upon heat shock in vitro. Purified $\mathrm{Cdc} 19^{\mathrm{WT}}, \mathrm{Cdc} 19^{\text {irrev }}$ and a non-aggregating $\mathrm{Cdc} 19$ mutant as negative control $\left(\mathrm{Cdc} 19^{\triangle \mathrm{PEP}}[1]\right)$ were incubated with ThT $(\mathbf{B})$ or Congo Red $(\mathbf{C})$ at $4{ }^{\circ} \mathrm{C}$ or after a 10 min heat shock at $42{ }^{\circ} \mathrm{C}$, and fluorescence emission was measured at $490 \mathrm{~nm}$ or $614 \mathrm{~nm}$, respectively. The graphs show the mean \pm S.E.M. and individual data points of three independent experiments (statistical analysis with two-tailed t-test, for ThT: $P_{W T}<0.0001, P_{\text {irrev }}$ $=0.0091$, for Congo Red: $\left.P_{W T}=0.0003, P_{\text {irrev }}=0.0128\right)$.

(D) In vivo-formed $\mathrm{Cdc} 19^{\mathrm{WT}}$ and $\mathrm{Cdc} 19^{\text {irrev }}$ aggregates are stained with Congo Red. Cells expressing GFP-tagged Cdc19 ${ }^{\mathrm{WT}}$ or $\mathrm{Cdc} 19^{\text {irrev }}$ were harvested when exponentially growing or after a 30 min heat shock at $42{ }^{\circ} \mathrm{C}$. Cells were gently lysed as described in Materials and Methods, Cdc19-GFP was immobilized in a GFP-trap microfluidic chamber and then stained with Congo Red. GFP (left column) and the Congo Red signal (middle column) were detected by fluorescence microscopy, and merged to visualize co-localization (right column). Cdc19GFP aggregates stained with Congo Red are marked with arrow heads. Images are representative of three independent experiments.

(E) Limited-Proteolysis Mass Spectrometry (LiP-MS) results indicate that $\mathrm{Cdc} 19^{\mathrm{WT}}$ and $\mathrm{Cdc} 19^{\text {irrev }}$ undergo comparable structural transitions upon aggregation in vitro and in vivo. Recombinant $\mathrm{Cdc} 19^{\mathrm{WT}}$ or $\mathrm{Cdc} 19^{\text {irrev }}$ were purified and soluble $\mathrm{Cdc} 19$ or heat shock-induced Cdc19 aggregates $\left(10\right.$ min at $\left.42{ }^{\circ} \mathrm{C}\right)$ were analysed by LiP-MS as described in Material and Methods. Volcano plots were used to compare Cdc19 peptides detected in soluble and aggregated $\mathrm{Cdc} 19^{\mathrm{WT}}$ and Cdc19 $9^{\text {irrev }}$, and upregulated (red) or downregulated (blue) conformation-sensitive peptides are highlighted. To compare these in vitro data with in vivo conformations, cells expressing Cdc $19^{\text {WT }}$ GFP or Cdc1 $19^{\text {irrev }}$ GFP were harvested during exponential growth or stationary phase 

described in Material and Methods. The volcano plots were used to compare Cdc19 peptides detected during exponential growth and starvation, and upregulated (red) or downregulated (blue) conformation-sensitive peptides are highlighted. The conformation-specific LiP-MSpeptides detected in the in vitro and in vivo experiments were mapped to the Cdc19 protein, schematically represented with the functional domains as described in panel A. Note that the conformation-specific LiP-MS peptides are comparable between wild-type and the Cdc19 ${ }^{\text {irrev }}$ mutant, with similar aggregate structures assembled in vitro upon heat shock and in vivo upon starvation. 
Supplementary Figure 2 - Genetic screening identifies trehalose metabolism as a regulator of reversible Cdc19 aggregation

A Suppressor screening
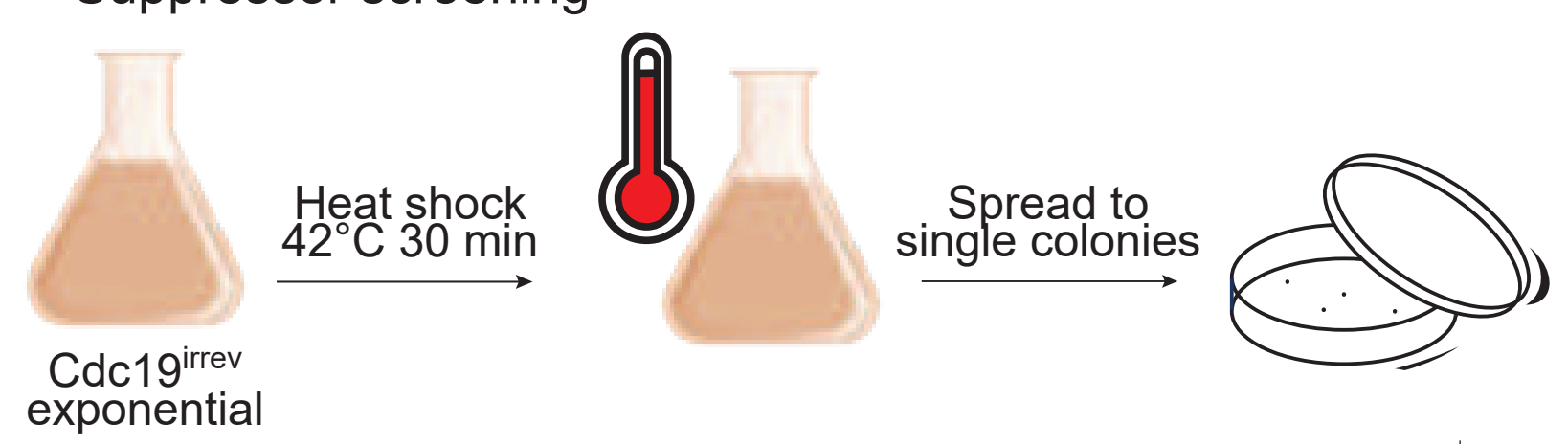

Isolate surviving single colonies

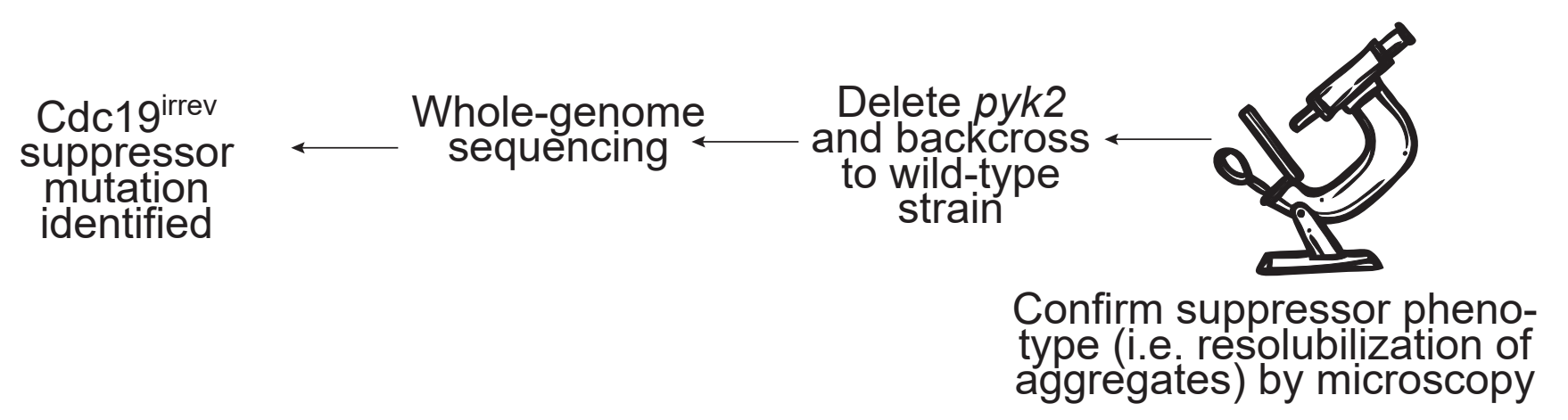

E

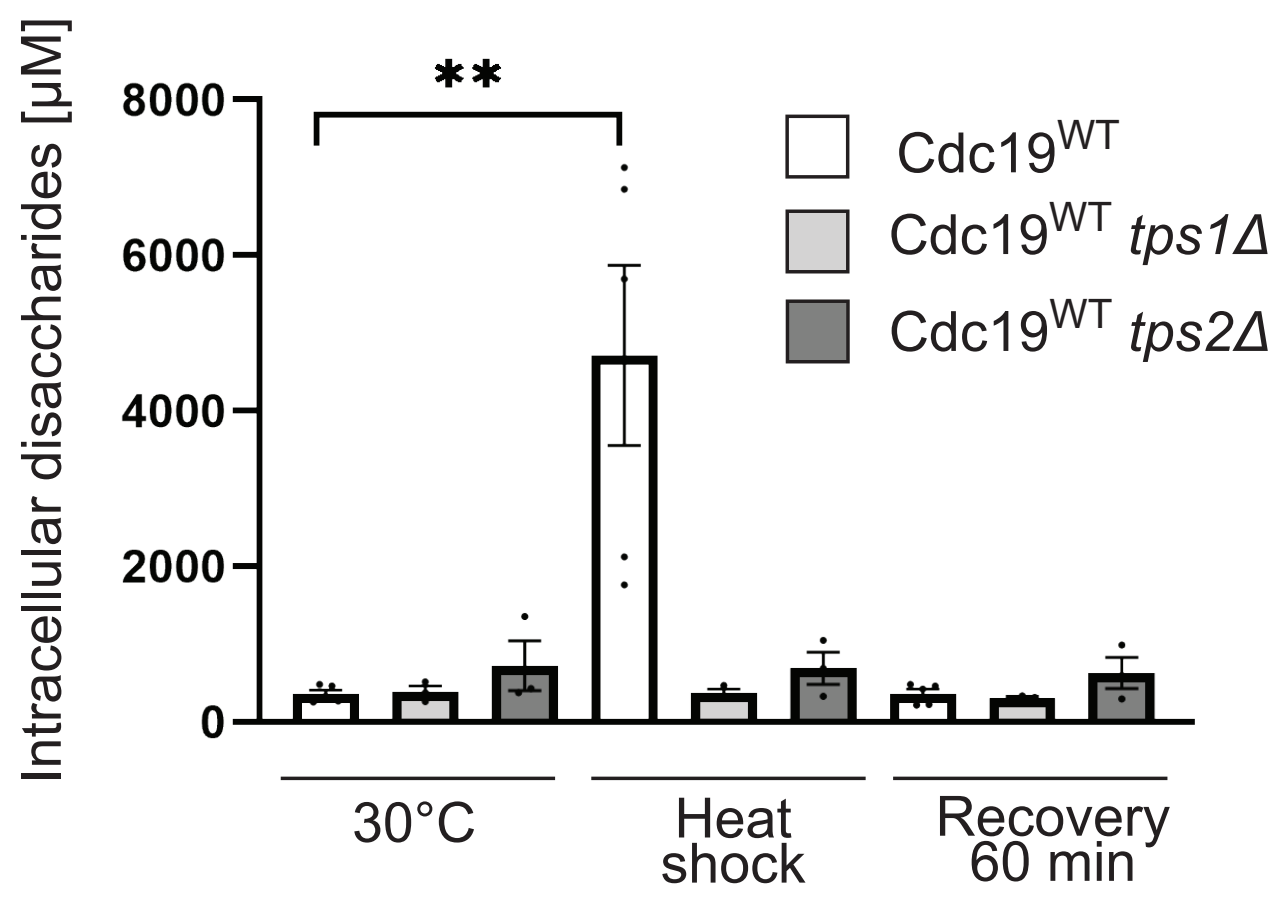

B Cdc19irrev suppressor mutation:

G386D

Tps3

$30^{\circ} \mathrm{C} \quad 42^{\circ} \mathrm{C} 30$ min then $30^{\circ} \mathrm{C}$

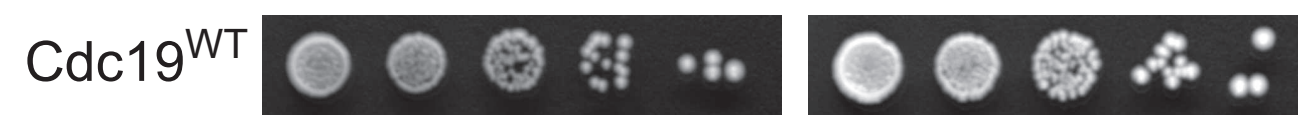

Cdc19irrev $\bigcirc>0$

Cdc19irrev
$\operatorname{tps} 3-G 386 D$

C

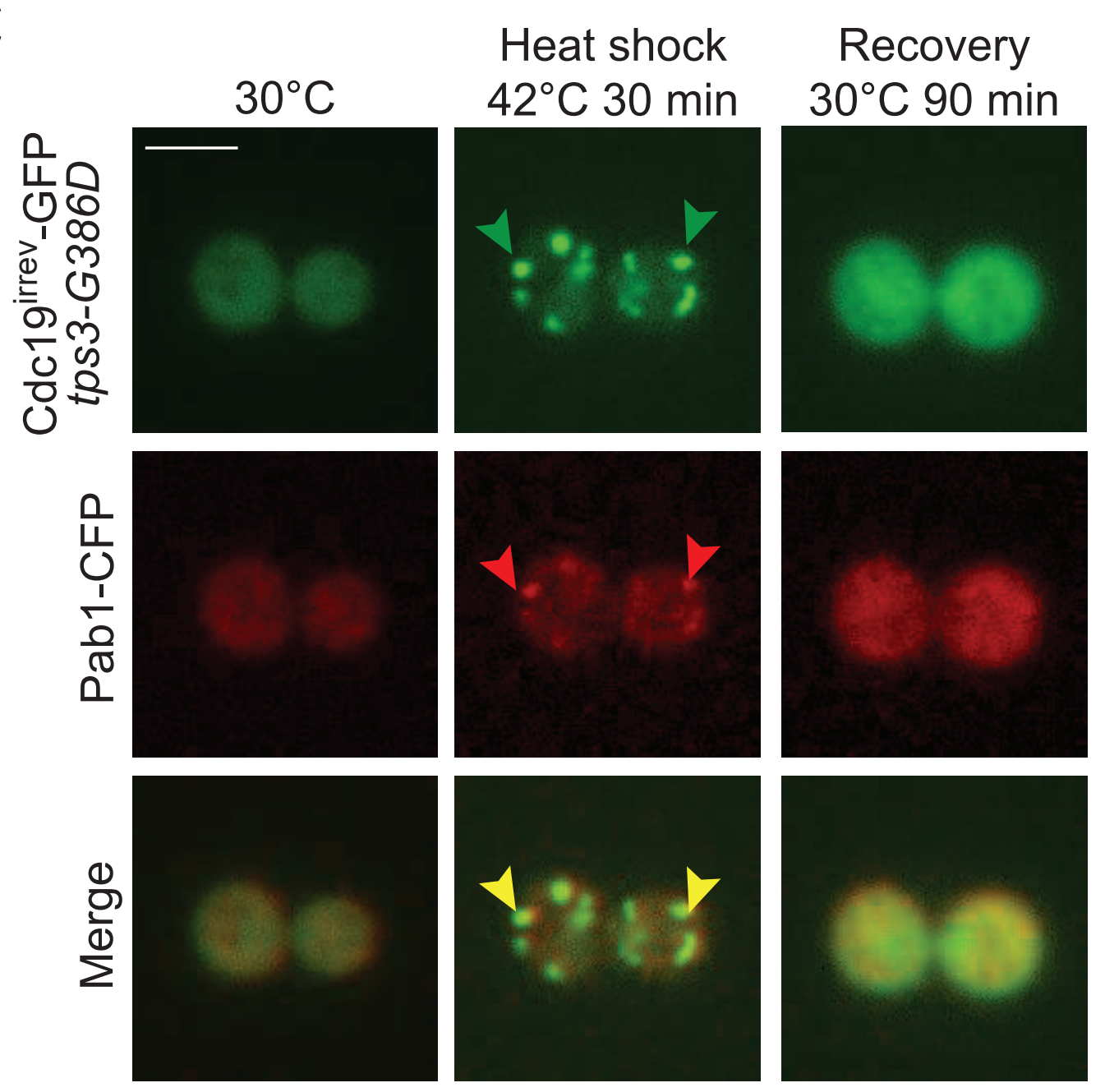

D

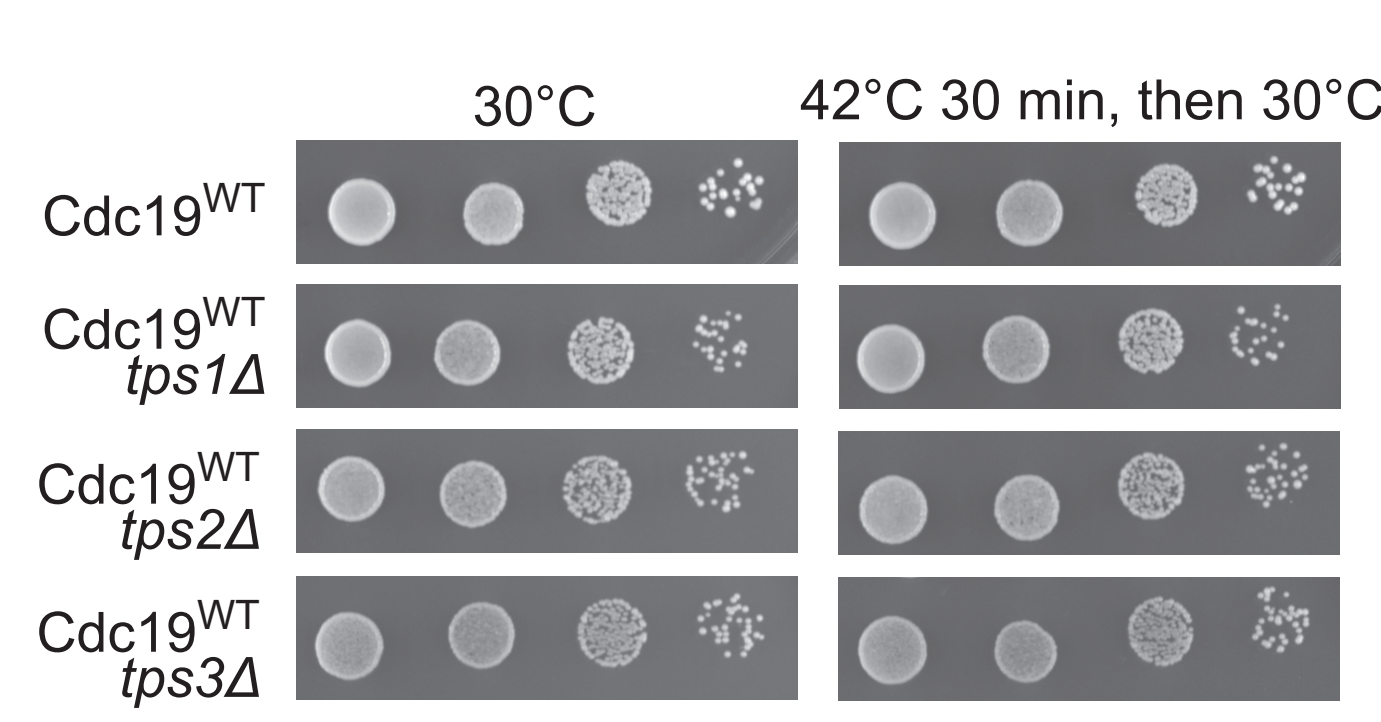

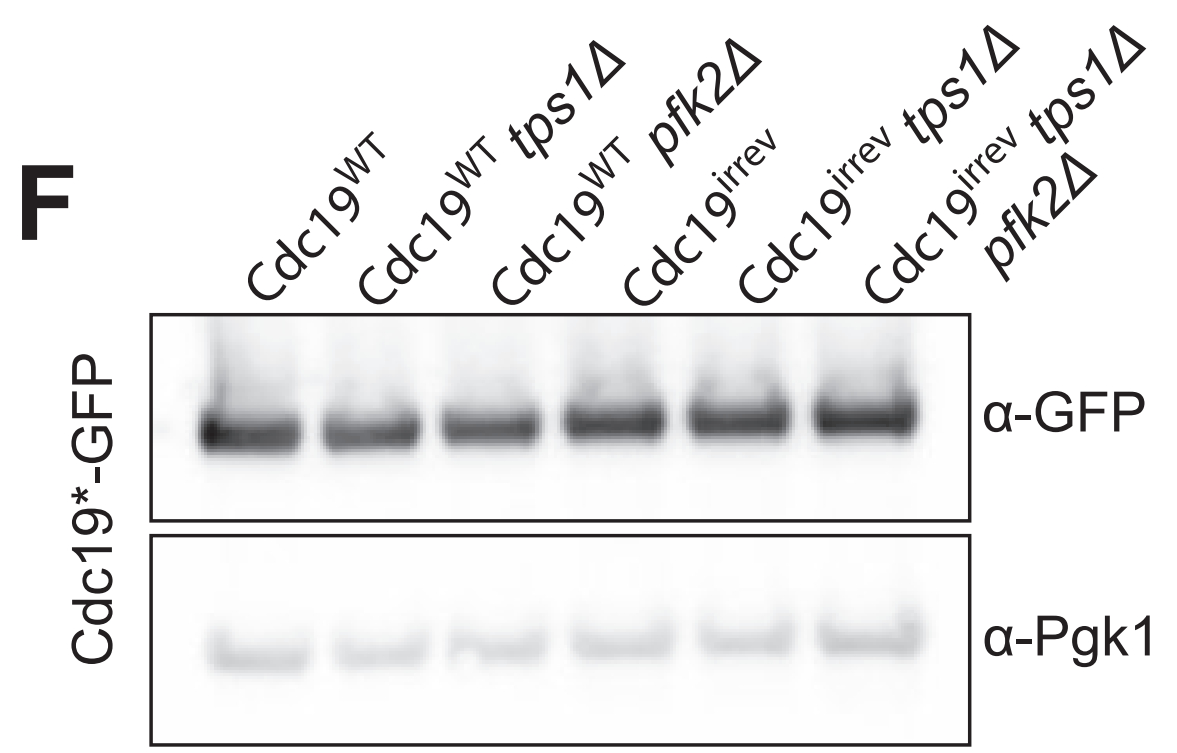

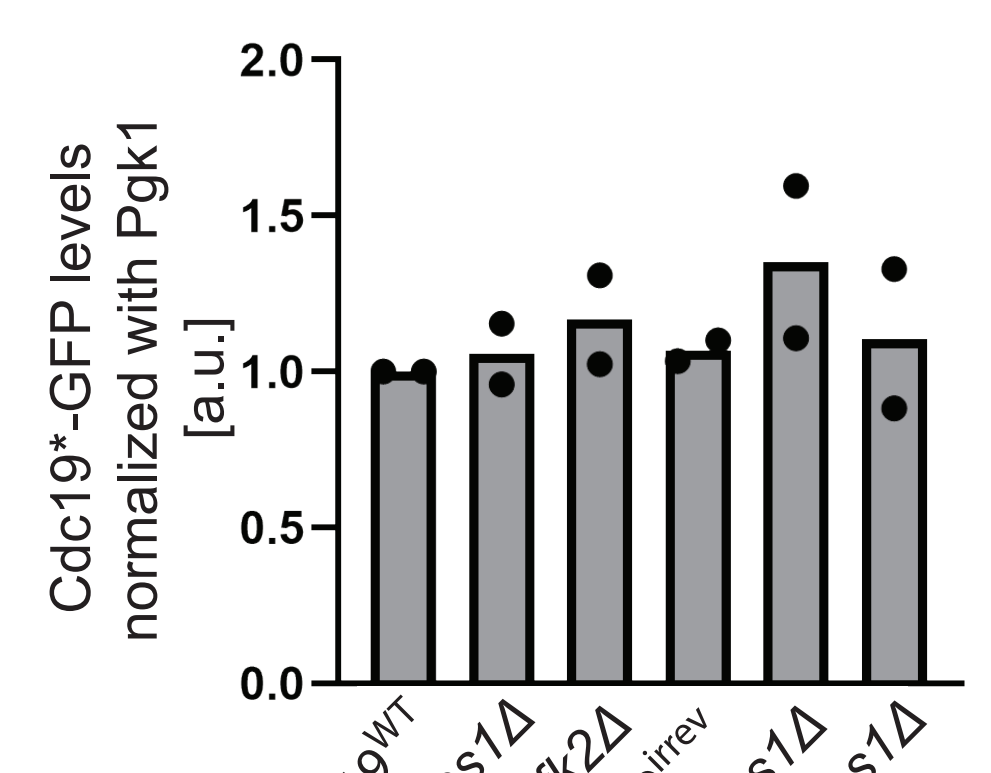

G

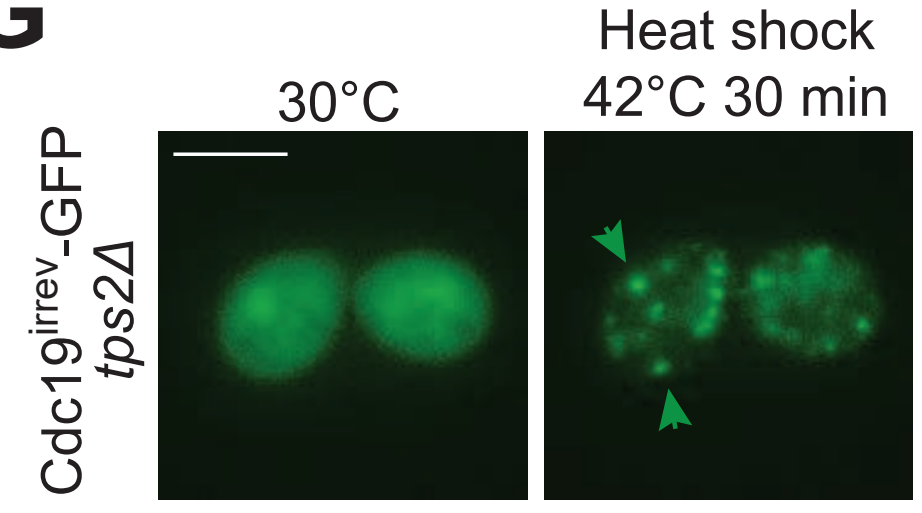

Recovery
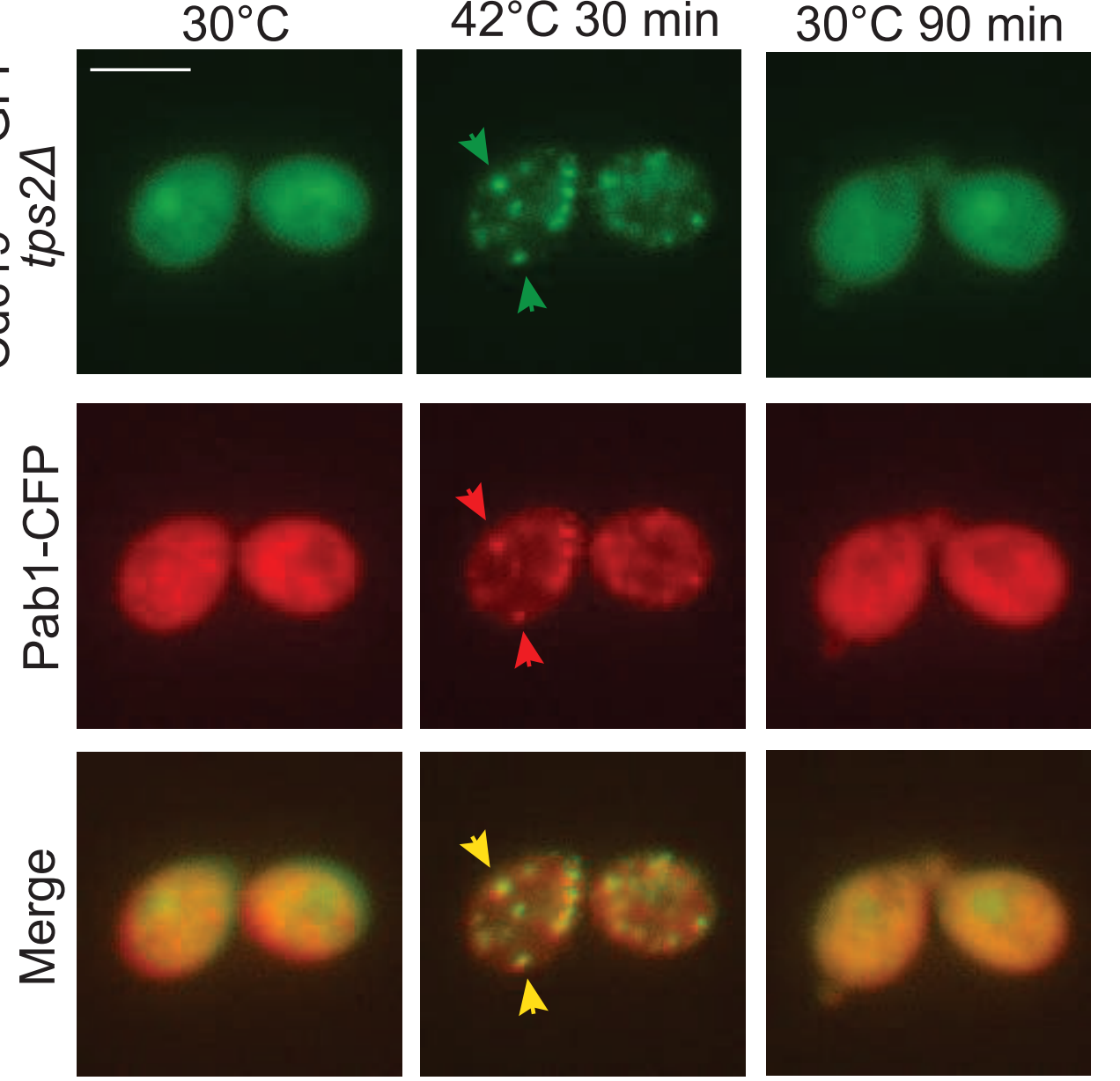

$\mathrm{H}$
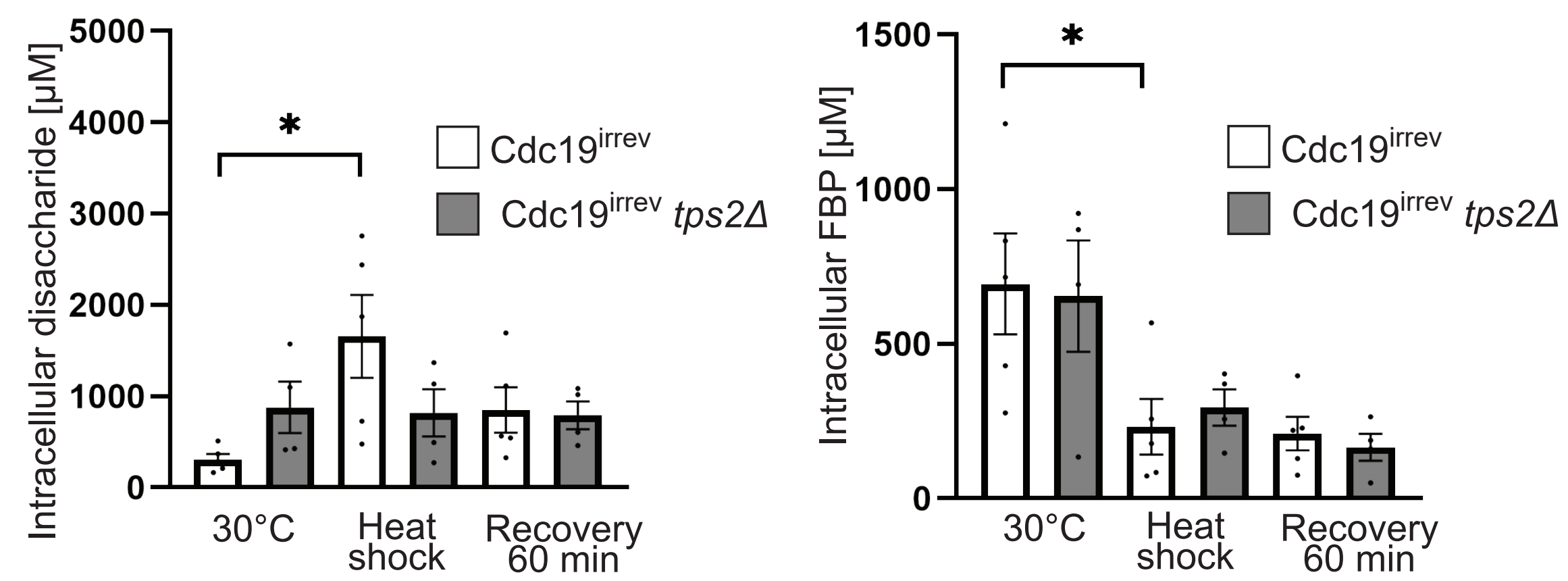


\section{Suppl. Figure 2: Genetic screening identifies trehalose metabolism as a regulator of reversible}

\section{Cdc19 aggregation}

(A) Schematic representation of the screening protocol used to identify suppressors of stressinduced growth arrest in Cdc19 $9^{\text {irrev }}$ cells. $c d c 19^{\text {irrev }}$ cells were subjected to a 30 min heat shock at $42{ }^{\circ} \mathrm{C}$, then plated at $30^{\circ} \mathrm{C}$, and the few spontaneous suppressors which could resume growth after stress were isolated. $p y k 2$ was deleted in these cells and single gene suppressors still able to restart cell growth after stress were further characterized. Whole-genome sequencing was performed to identify responsible mutations.

(B) The $c d c 19^{\text {irrev }}$ suppressor screen identified a single point mutation (G385D) in the tps3 gene encoding for a subunit of trehalose synthase. Serial dilutions of exponentially growing $c d c 19^{W T}$, $c d c 19^{\text {irrev }}$ and $c d c 19^{\text {irrev }}$ tps3-G385D were spotted on agar plates before (left) or after a $30 \mathrm{~min}$ heat shock of $42{ }^{\circ} \mathrm{C}$ (right). The plates were imaged after 3 days at $30{ }^{\circ} \mathrm{C}$.

(C) Exponentially growing tps3-G385D cells expressing GFP-tagged Cdc19 $9^{\text {irrev }}$ and the SG marker Pab1-CFP were grown in a microfluidic chip at $30{ }^{\circ} \mathrm{C}$ in SD-full medium (left column), heat shocked for $30 \mathrm{~min}$ at $42{ }^{\circ} \mathrm{C}$ (middle column) and allowed to recover at $30{ }^{\circ} \mathrm{C}$ (right column). Representative GFP- (top row) and CFP-images (middle row) were taken at the indicated time points, and merged (bottom row) to visualize co-localization of Cdc19 $9^{\text {irrev }}$ aggregates and SGs (arrow heads). Scale bar: $5 \mu \mathrm{m}$.

(D) Serial dilutions of exponentially growing wild-type, $\operatorname{tps} 1 \Delta$, $\operatorname{tps} 2 \Delta$, and tps $3 \Delta$ cells were spotted on agar plates before (left) or after a 30 min heat shock of $42{ }^{\circ} \mathrm{C}$ (right). The plates were imaged after 3 days at $30^{\circ} \mathrm{C}$.

(E) Wild-type cells accumulate high levels of disaccharides upon heat shock, which almost exclusively consist of trehalose [2]. Deletion of either tps1 or tps2 abolishes this increase. Intracellular disaccharide concentrations $(\mu \mathrm{M})$ were measured by mass-spectrometry in wildtype, tps $1 \Delta$, and tps $2 \Delta$ cells at the indicated time points before, during and after a 30 min heat shock at $42{ }^{\circ}$ C. Mean \pm S.E.M. determined by five (for wild-type) and three (for tps $1 \Delta$ and 
$\operatorname{tps} 2 \Delta$ ) independent experiments are shown (statistical analysis with Mann-Whitney test, $P=$ $0.0079)$.

(F) Extracts prepared from wild-type, $t p s 1 \Delta, p f k 2 \Delta$ or $t p s 1 \Delta p f k 2 \Delta$ expressing either GFP-tagged Cdc19 ${ }^{\mathrm{WT}}$ or Cdc19 $9^{\text {irrev }}$ were immunoblotted with antibodies against GFP (left panel) or for control Pgk1 (right panel). The mean Cdc19-GFP levels normalized with Pgk1 are shown ( $\mathrm{n}=$ 2). Note that neither tps $1 \Delta$ nor $p f k 2 \Delta$ significantly alter Cdc19 protein levels.

(G) Exponentially growing tps $2 \Delta$ cells expressing GFP-tagged Cdc $19^{\text {irrev }}$ and the SG marker Pab1CFP were grown in a microfluidic chip at $30{ }^{\circ} \mathrm{C}$ in SD-full medium (left column), heat shocked for $30 \mathrm{~min}$ at $42{ }^{\circ} \mathrm{C}$ (middle column) and allowed to recover at $30{ }^{\circ} \mathrm{C}$ (right column). Representative GFP- (top row) and CFP-images (middle row) were taken at the indicated time points, and merged (bottom row) to visualize co-localization of Cdc19 $9^{\text {irrev }}$ aggregates and SGs (arrow heads). Scale bar: $5 \mu \mathrm{m}$.

(F) Intracellular disaccharide (left) and FBP (right) concentrations $(\mu \mathrm{M})$ were measured by massspectrometry in $c d c 19^{\text {irrev }}$ and $c d c 19^{\text {irrev }}$ tps $2 \Delta$ strains at the indicated time points before, during and after a 30 min heat shock at $42{ }^{\circ} \mathrm{C}$. Mean \pm S.E.M. determined by four (for $c d c 19^{\text {irrev }} \operatorname{tps} 2 \Delta$ ) and five (for $c d c 19^{\text {irrev }}$ ) independent experiments are shown (statistical analysis with MannWhitney test, $\left.P_{\text {Disaccharides }}=0.0159, P_{F B P}=0.0317\right)$. Note that intracellular disaccharides in yeast almost exclusively consist of trehalose [2], and that $\operatorname{cdc} 19^{\text {irrev }} \operatorname{tps} 2 \Delta$ have low trehalose and low FBP levels during and after stress. 


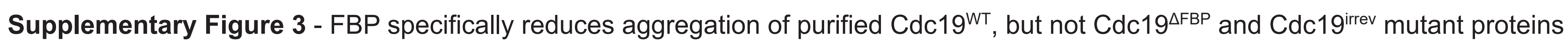

A
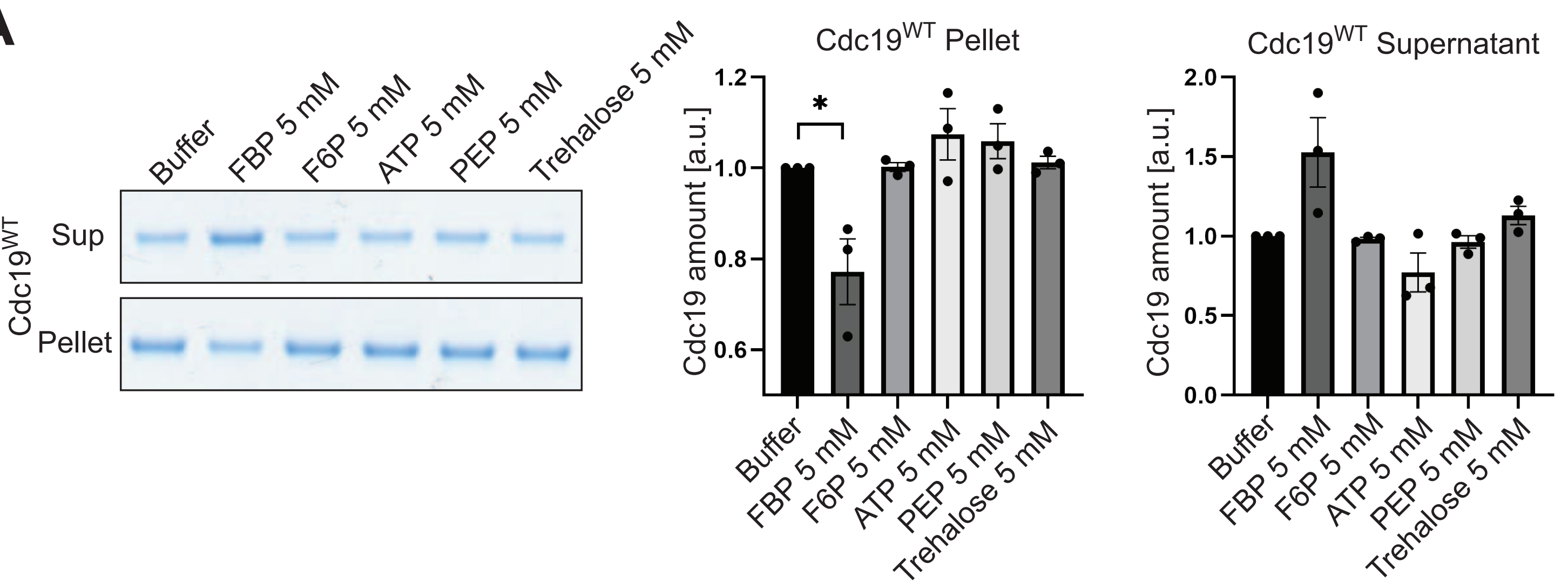

B

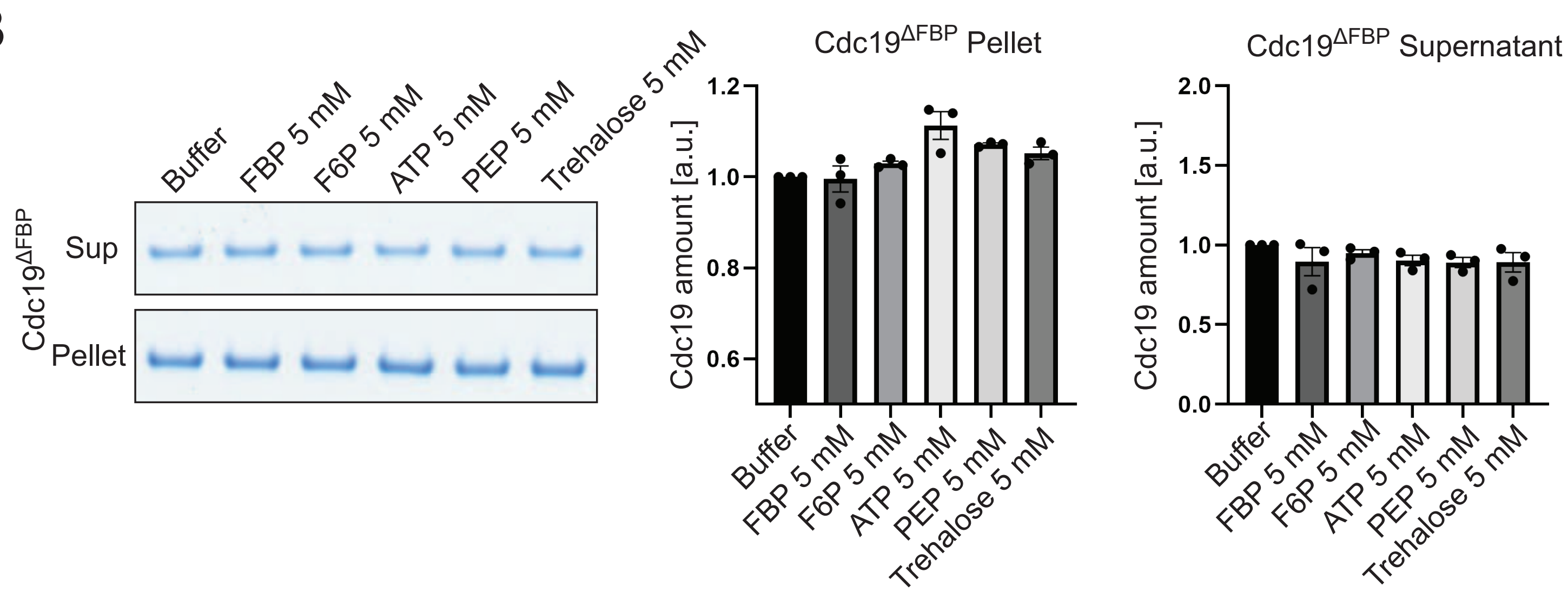

C

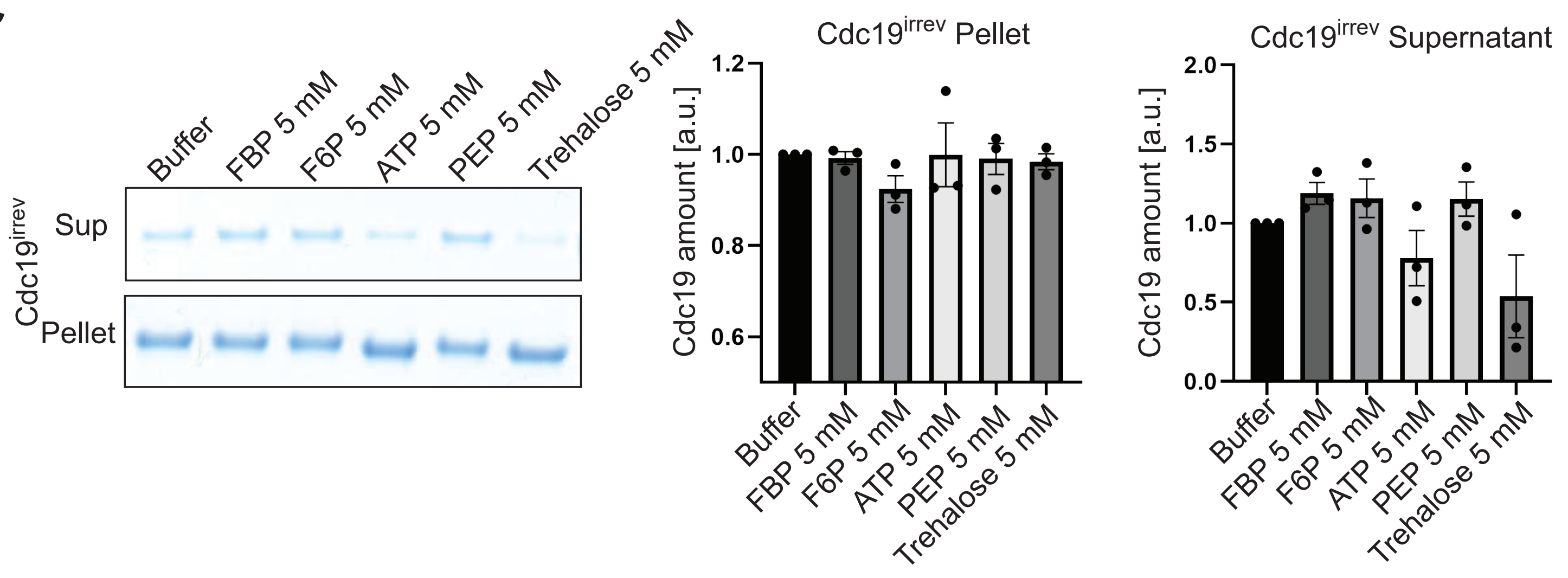

D

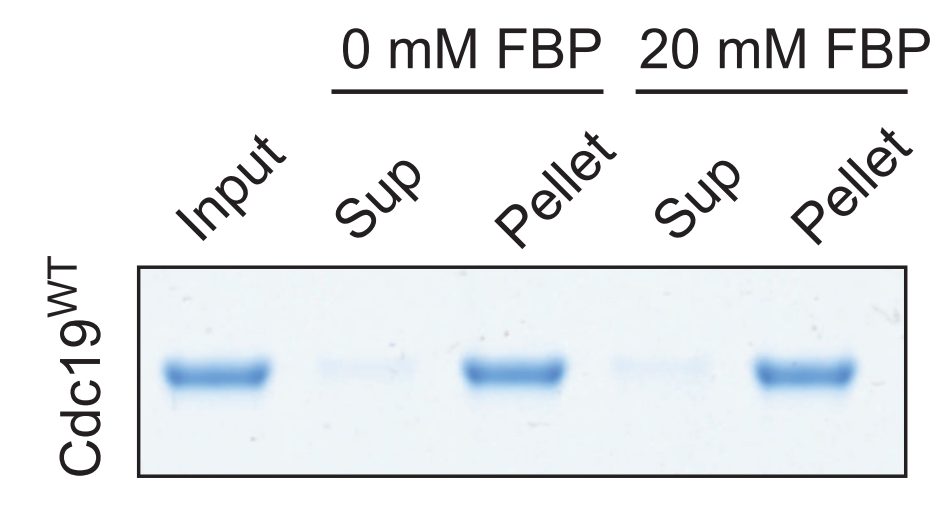


Suppl. Figure 3: FBP specifically reduces aggregation of purified Cdc19 ${ }^{\mathrm{WT}}$, but not $\mathrm{Cdc19}^{\mathrm{AFBP}}$ and Cdc19 ${ }^{\text {irrev }}$ mutant proteins

(A) - (C) Purified wild-type Cdc19 (A), FBP binding-deficient Cdc19 $9^{\triangle \mathrm{FBP}}$ mutant (B) or Cdc19 $9^{\text {irrev }}$ mutant $(\mathbf{C})$ proteins were mixed as indicated with $5 \mathrm{mM}$ FBP or for control $5 \mathrm{mM} \mathrm{F6P,} 5 \mathrm{mM}$ ATP, $5 \mathrm{mM}$ PEP, $5 \mathrm{mM}$ trehalose or buffer and incubated at $30{ }^{\circ} \mathrm{C}$ for 14 hours. Cdc19 aggregates were separated from soluble protein by centrifugation and the supernatant (Sup, containing soluble Cdc19) and pellet (Pellet, containing aggregated Cdc19) fractions analysed by SDS-PAGE and Coomassie blue staining (left gels). Cdc19 amount was quantified in the Pellet (left graphs) and Supernatant (right graphs) by measuring Cdc19 band intensity using ImageJ and normalizing to buffer controls, and is displayed as mean and S.E.M. from three independent experiments (statistical analysis with two-tailed t-test, $P=0.0342$ ). Note that, in contrast to $\mathrm{Cdc} 19^{\triangle \mathrm{FBP}}$ and $\mathrm{Cdc} 19^{\text {irrev }}, \mathrm{FBP}$ reduces aggregation of $\mathrm{Cdc} 19^{\mathrm{WT}}$, while all other tested metabolites have no effect on aggregation.

(D) Addition of FBP alone is not sufficient to re-solubilize pre-formed $\mathrm{Cdc} 19^{\mathrm{WT}}$ amyloids in vitro. Purified Cdc1 $19^{\mathrm{WT}}$ (Input) was incubated for $10 \mathrm{~min}$ at $42{ }^{\circ} \mathrm{C}$ to trigger its aggregation, and the resulting amyloids were incubated with $(20 \mathrm{mM})$ or without $(0 \mathrm{mM})$ FBP for several hours. Cdc19 re-solubilization was then tested by centrifugation and analysis of the resulting supernatant (Sup) and pellet (Pellet) fractions by SDS-PAGE and Coomassie blue staining. The image is representative of three independent experiments. 
Supplementary Figure 4 - The chaperones Hsp104 and Ssa2 co-operate with FBP to efficiently disassemble Cdc19 amyloids in vivo

A
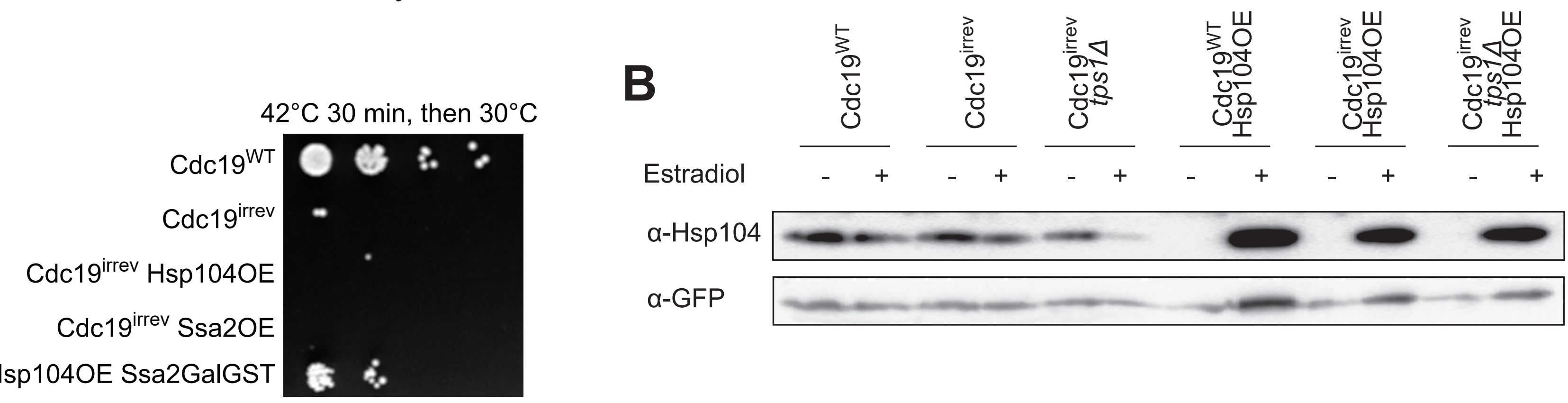

Cdc19irrev Hsp104OE Ssa2GalGST ef

C

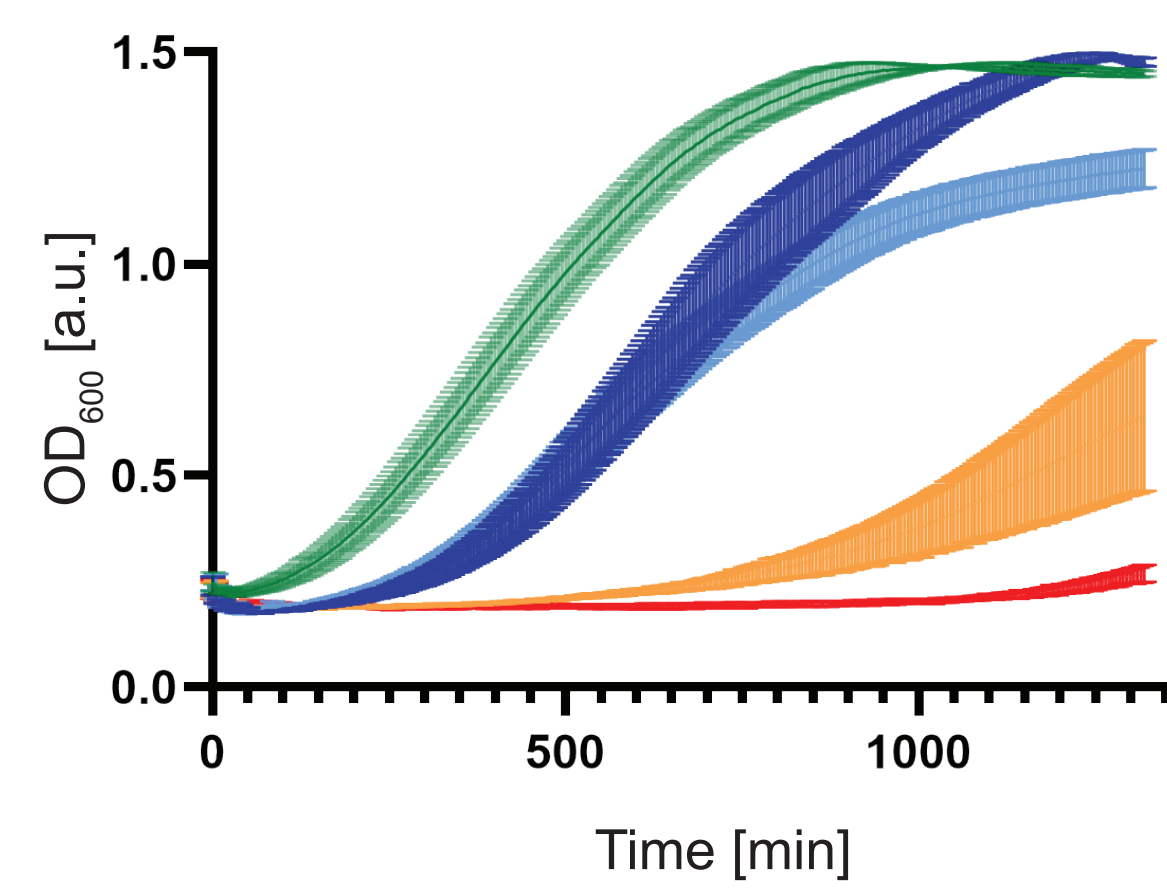

Cdc19WT

- Cdc19irrev tps1 $1 \Delta$ Hsp104OE

- Cdc19irrev tps1

- Cdc19irrev Hsp104OE

- Cdc19irrev

D
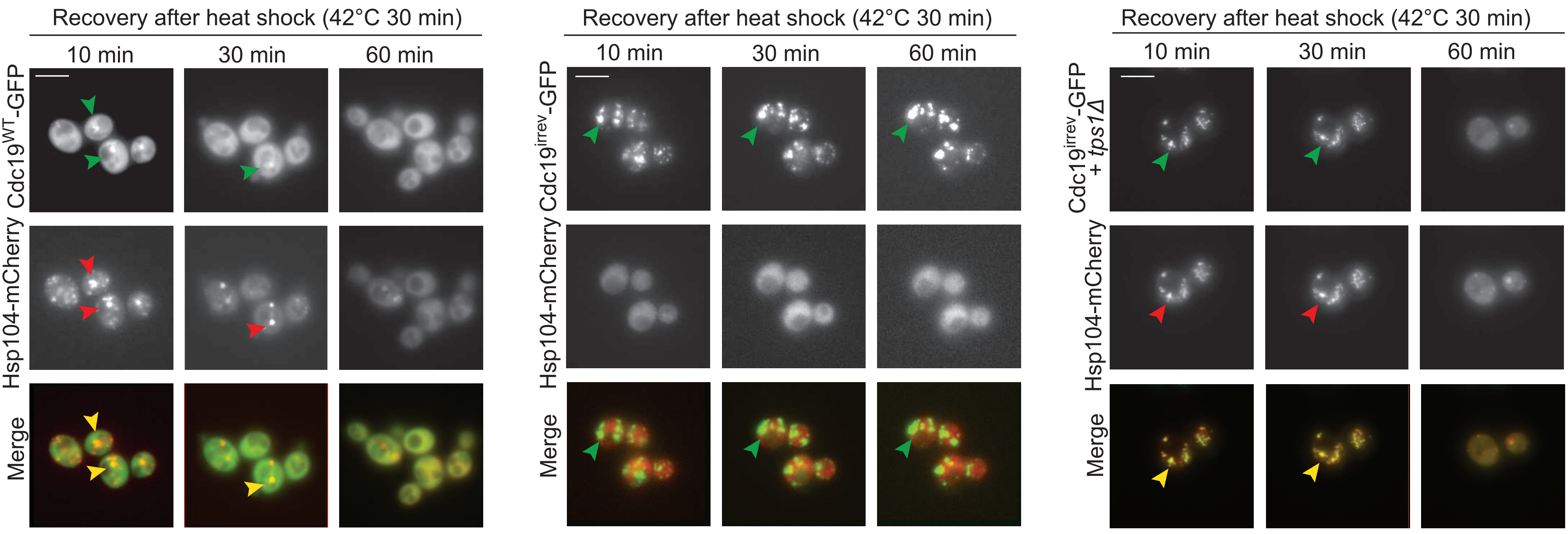


\section{Suppl. Figure 4: The chaperones Hsp104 and Ssa2 co-operate with FBP to efficiently}

\section{disassemble Cdc19 amyloids in vivo}

(A) Co-overexpression of Hsp104 and Ssa2 partially restores growth of $c d c 19^{\text {irrev }}$ cells after heat stress. $c d c 19^{W T}$ and $c d c 19^{\text {irrev }}$ cells overexpressing as indicated Hsp104 or Ssa2, or both together by addition of $10 \mathrm{mM}$ estradiol for 3 hours were treated for $30 \mathrm{~min}$ at $42{ }^{\circ} \mathrm{C}$. Serial dilutions were spotted on agar plates and grown at $30{ }^{\circ} \mathrm{C}$ for 3 days. The image is representative of three independent experiments.

(B) Hsp104 protein levels were quantified in the indicated strains by Western blot either in the absence (-) or presence (+) of $10 \mathrm{mM}$ estradiol for 3 hours. The image is representative of three independent experiments.

(C) Increased FBP levels and Hsp104 co-operate to efficiently restart growth after stress in $c d c 19^{\text {irrev }}$ cells. Exponentially growing wild-type or tps $1 \Delta$ cells expressing either $\mathrm{Cdc} 19^{\mathrm{WT}}$ or $\mathrm{Cdc} 19^{\text {irrev }}$ were subjected to a $30 \mathrm{~min}$ heat shock at $42{ }^{\circ} \mathrm{C}$. Where indicated, overexpression of Hsp104 was induced by treating cells with $10 \mathrm{mM}$ estradiol for 3 hours. Growth restart after stress release of the indicated strains was quantified by measuring the cell density (OD600) over time after inoculation of equal cell numbers at $30{ }^{\circ} \mathrm{C}$. Mean cell density 22 hours after stress release is shown, with error bars representing S.E.M. from three independent experiments. Note that Hsp104 overexpression and increased FBP levels co-operate to rescue $c d c 19^{\text {irrev }}$ cells after heat shock.

(D) Wild-type (left and middle panels) or tps1 $\Delta$ (right panels) cells co-expressing mCherry-tagged Hsp104 and either GFP-tagged Cdc19 ${ }^{\mathrm{WT}}$ (left panel) or Cdc19 ${ }^{\text {irrev }}$ mutant (middle and right panels) were heat stressed for $30 \mathrm{~min}$ at $42{ }^{\circ} \mathrm{C}$ and imaged by fluorescence microscopy at the times indicated (minutes). Representative GFP- (left row) and mCherry-images (middle row) are shown, together with the merged image (bottom row) to visualize co-localization of Cdc19 aggregates and Hsp104. Images are representative of three independent experiments. Scale bar: $5 \mu \mathrm{m}$. 
1166 Suppl. Table S1: Yeast strains used in this study

\begin{tabular}{|c|c|c|}
\hline Strain name & Genotype & Source \\
\hline$c d c 194+$ Pyk2OE & BY4741, cdc19A::NAT ${ }^{R} ; p R S 416-p T E F 1-P Y K 2$ & Saad et al. \\
\hline Cdc19WT_GFP & BY4741, CDC19::CDC19-GFP-HIS3 & Invitrogen \\
\hline Cdc19WT_GFP + tps $1 \Delta$ & BY4741, CDC19::CDC19-GFP-HIS3; tps1 $1:: K A N^{R}$ & This study \\
\hline Cdc19WT_GFP + tps $2 \Delta$ & BY4741, CDC19::CDC19-GFP-HIS3; tps2 $2: \because K A N^{R}$ & This study \\
\hline 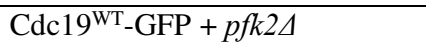 & BY4741, CDC19::CDC19-GFP-HIS3; pfk2A::KAN & This study \\
\hline Cdc19WT_GFP + Pyk2OE & BY4741, CDC19::CDC19-GFP-HIS3; pRS416-pTEF1-PYK2 & Saad et al. \\
\hline $\begin{array}{l}\text { Cdc19WT_GFP }+ \text { Hsp104- } \\
\text { mCherry }\end{array}$ & BY4741, CDC19::CDC19-GFP-HIS3; HSP104::HSP104-mCherry & This study \\
\hline 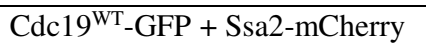 & BY4741, CDC19::CDC19-GFP-HIS3; SSA2::SSA2-mCherry & This study \\
\hline Cdc19 $19^{\text {irrev-GFP }}$ & BY4741, CDC19::CDC19-T372D, T376D, S377D, S385D-GFP-HIS3 & Saad et al. \\
\hline 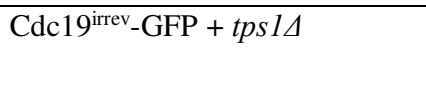 & $\begin{array}{l}\text { BY4741, CDC19::CDC19-T372D, } \\
\text { tps1 } 14:: \mathrm{KAN}^{\mathrm{R}}\end{array}$ & This study \\
\hline Cdc19 $19^{\text {irrev }}-\mathrm{GFP}+t p s 1 \Delta+p f k 2 \Delta$ & $\begin{array}{l}\text { BY4741, CDC19::CDC19-T372D, T376D, S377D, } \\
\text { tps } 1 \Delta:: N A T^{R} ; \text { pfk } 2 \Delta:: K A N^{R}\end{array}$ & This study \\
\hline Cdc19 $9^{\text {irrev }}-\mathrm{GFP}+$ Pyk2OE & $\begin{array}{l}\text { BY4741, CDC19::CDC19-T372D, T376D, S377D, S385D-GFP-HIS3; pRS416- } \\
\text { pTEF1-PYK2 }\end{array}$ & This study \\
\hline $\begin{array}{l}\text { Cdc19 } 9^{\text {irrev }} \text {-GFP } \\
\text { suppressor }\end{array}$ & $\begin{array}{l}\text { BY4741, CDC19::CDC19-T372D, T376D, S377D, S385D-GFP-HIS3; tps3- } \\
\text { G386D }\end{array}$ & This study \\
\hline Cdc19 $9^{\text {irrev }}-\mathrm{GFP}+\operatorname{tps} 3 \Delta$ & 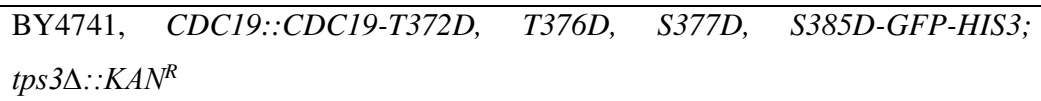 & This study \\
\hline Cdc19 irrev-GFP + tps $2 \Delta$ & $\begin{array}{l}\text { BY4741, CDC19::CDC19-T372D, } \quad \text { T376D, S377D, } \\
\operatorname{tps} 2 \triangle:: K A N^{R}\end{array}$ & This study \\
\hline 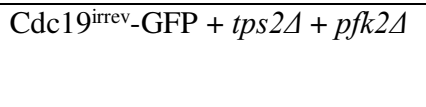 & $\begin{array}{l}\text { BY4741, CDC19::CDC19-T372D, T376D, S377D, S385D-GFP-HIS3; } \\
\operatorname{tps} 2 \Delta:: N A T^{R} ; p f k 2 \Delta:: K A N^{R}\end{array}$ & This study \\
\hline $\mathrm{Cdc} 19^{\mathrm{irrev}}-\mathrm{GFP}+\operatorname{tps} 1 \Delta+\operatorname{tps} 2 \Delta$ & $\begin{array}{l}\text { BY4741, CDC19::CDC19-T372D, } \quad \text { T376D, } \\
\text { tps } 1 \Delta 3:: K A N R ; \text { tps } 2 \Delta:: N A T^{R}\end{array}$ & This study \\
\hline $\begin{array}{l}\text { Cdc19 }{ }^{\text {irrev-GFP }}+\text { Hsp104- } \\
\text { mCherry }\end{array}$ & $\begin{array}{l}\text { BY4741, CDC19::CDC19-T372D, T376D, S377D, } \\
\text { HSP104::HSP104-mCherry }\end{array}$ & This study \\
\hline Cdc19 $9^{\text {irrev_GFP }+ \text { Ssa2-mCherry }}$ & $\begin{array}{l}\text { BY4741, CDC19::CDC19-T372D, } \\
\text { SSA2::SSA2-mCherry }\end{array}$ & This study \\
\hline $\begin{array}{l}\text { Cdc19 irrev-GFP }+\operatorname{tps} 1 \Delta+ \\
\text { Hsp104-mCherry }\end{array}$ & $\begin{array}{l}\text { BY4741, CDC19::CDC19-T372D, T376D, S377D, S385D-GFP-HIS3; } \\
\text { tps 1 }::: K A N^{R} ; \text { HSP104::HSP104-mCherry }\end{array}$ & This study \\
\hline $\begin{array}{l}\text { Cdc19 }{ }^{\text {irrev }}-\text { GFP }+\operatorname{tps} 1 \Delta+\text { Ssa2- } \\
\text { mCherry }\end{array}$ & $\begin{array}{l}\text { BY4741, CDC19::CDC19-T372D, } \\
\text { tps } 1 \Delta:: K A N^{R} ; \text { SSA2::SSA2-mCherry }\end{array}$ & This study \\
\hline Cdc19 ${ }^{\triangle \mathrm{FBP}}$-GFP & BY4741, CDC19::CDC19-T402E-GFP-HIS3 ; pRS416-pTEF1-PYK2 & Saad et al. \\
\hline $\mathrm{Cdc} 19^{\Delta \mathrm{FBP}}-\mathrm{GFP}+t p s 1 \Delta$ & $\begin{array}{l}\text { BY4741, CDC19::CDC19-T402E-GFP-HIS3 } \\
\text { tps } 1 \Delta:: \mathrm{KAN}^{\mathrm{R}}\end{array}$ & This study \\
\hline $\begin{array}{l}\text { Cdc19 }{ }^{\triangle \mathrm{FBP}}-\mathrm{GFP}+\mathrm{Hsp} 104- \\
\text { mCherry }\end{array}$ & $\begin{array}{l}\text { BY4741, CDC19::CDC19-T402E-GFP-HIS3 } \\
\text { HSP104::HSP104-mCherry }\end{array}$ & This study \\
\hline $\begin{array}{l}\text { Cdc19 }{ }^{\triangle \mathrm{FBP}} \text {-GFP }+ \text { tps14 }+ \\
\text { Hsp104-mCherry }\end{array}$ & 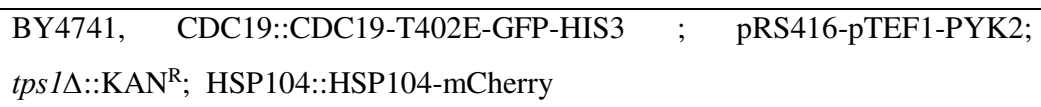 & This study \\
\hline
\end{tabular}




\begin{tabular}{|c|c|c|}
\hline Cdc19 ${ }^{\text {Not-agg_GFP }}$ & BY4741, CDC19::CDC19-R49A-GFP- HIS3; pRS416-pTEF1-PYK2 & Saad et al. \\
\hline Cdc19 $9^{\text {irrev-GFP + Hsp104OE }}$ & $\begin{array}{lllll}\text { BY4741, CDC19::CDC19-T372D, } & \text { T376D, } & \text { S377D, } & \text { S385D-GFP-HIS3 } \\
\text { HSP104::HSP104-mCherry } & & & \end{array}$ & This study \\
\hline Cdc19 $9^{\text {irrev }}-\mathrm{GFP}+\mathrm{Ssa} 2 \mathrm{OE}$ & $\begin{array}{l}\text { BY4741, CDC19::CDC19-T372D, } \quad \text { T376D, S377D, S385D-GFP-HIS3; } \\
\text { SSA2::SSA2-mCherry }\end{array}$ & This $\mathrm{s}$ \\
\hline $\begin{array}{l}\text { Cdc19 }{ }^{\text {irrev }}-\text { GFP }+ \text { Hsp104OE }+ \\
\text { Ssa2OE }\end{array}$ & $\begin{array}{l}\text { BY4741, CDC19::CDC19-T372D, T376D, S377D, S385D-GFP-HIS3; SSA2 Gal } \\
\text { GST; Hsp104OE }\end{array}$ & Thi \\
\hline $\begin{array}{l}\text { Cdc19 }{ }^{\text {irrev-GFP }}+\text { tps1 }+ \\
\text { Hsp104OE }\end{array}$ & $\begin{array}{l}\text { BY4741, CDC19::CDC19-T372D, } \quad \text { T376D, S377D, } \\
\text { tps1 S385D-GFP-HIS3; }\end{array}$ & Thi \\
\hline $\begin{array}{l}\text { Cdc19 }{ }^{\text {irrev-GFP }}+\text { tps1 }+ \\
\text { Hsp104OE }+ \text { SsaeOE }\end{array}$ & $\begin{array}{l}\text { BY4741, CDC19::CDC19-T372D, } \quad \text { T376D, S377D, } \\
\text { tps } 1 \triangle:: K A N^{R} ; H \operatorname{Hsp104OE}\end{array}$ & Thi \\
\hline+ Pab1-CFP & BY4741, CDC19::CDC19-GFP-HIS3; pRS415-Pab1-CFP & al. \\
\hline $\begin{array}{l}\text { Cdc19 } 19^{\mathrm{WT}}-\mathrm{GFP}+\text { tps } 1 \Delta+\text { Pab1- } \\
\text { CFP }\end{array}$ & CDC19::CDC19-GFP-HIS3; tps $1 \Delta: \because K A N^{R} ; p R S 415-P a b$ & \\
\hline $\begin{array}{l}\text { Cdc19 } 19^{\mathrm{WT}}-\mathrm{GFP}+\operatorname{tps} 2 \Delta+\text { Pabl- } \\
\text { CFP }\end{array}$ & $\overline{C D C 19:: C D C 19-G F P-H I S 3 ; t p s 2 \Delta:: K A N^{R} ; p R S 415-P a}$ & \\
\hline $\begin{array}{l}\mathrm{Cdc} 19^{\mathrm{WT}}-\mathrm{GFP}+p f k 2 \Delta+\text { Pab1- } \\
\text { CFP }\end{array}$ & $\overline{C D C 19:: C D C 19-G F P-H I S 3 ; p f k 2 \Delta: \because K A N^{R} ; p R S 415-P a b 1-C F P}$ & \\
\hline $\begin{array}{l}\text { Cdc19 }{ }^{\mathrm{WT}} \text {-GFP }+ \text { Pab1-CFP }+ \\
\text { Pyk2OE }\end{array}$ & BY4741 CDC19::CDC19-GFP-HIS3; pRS416-pTEF1-PYK2; $p R S 415-P$ & \\
\hline+ Pab1-CFP & $\begin{array}{l}\text { BY4741, CDC19::CDC19-T372D, T376D, S377D, S385D-GFP-HIS3; pRS415- } \\
\text { Pab1-CFP }\end{array}$ & \\
\hline $\begin{array}{l}\text { Cdc19 }{ }^{\text {Irrev }}-\mathrm{GFP}+\text { tps } 1 \Delta+\text { Pabl- } \\
\text { CFP }\end{array}$ & $\begin{array}{l}\text { BY4741, CDC19::CDC19-T372D, } \\
\text { tps } 1 \Delta:: K A N^{R} ; \text { pRS415-Pab1-CFP }\end{array}$ & \\
\hline 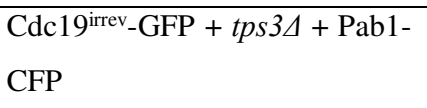 & $\begin{array}{l}\text { BY4741, CDC19::CDC19-T372D, } \quad \text { T376D, } \\
\text { tps3 } 34:: K A N^{R} ; \text { pRS415-Pab1-CFP }\end{array}$ & idy \\
\hline $\begin{array}{l}\text { Cdc19irrev-GFP + tps } 2 \Delta+\text { Pab1- } \\
\text { CFP }\end{array}$ & $\begin{array}{l}\text { BY4741, CDC19::CDC19-T372D, T376D, S377D, S385D-GFP-HIS3; } \\
\operatorname{tps} 2 \triangle:: K A N^{R} ; \text { pRS415-Pab1-CFP }\end{array}$ & ady \\
\hline 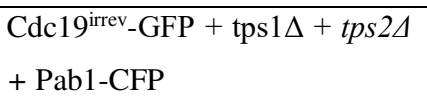 & $\begin{array}{l}\text { BY4741, CDC19::CDC19-T372D, T376D, S377D, } \\
\text { tps } 1 \Delta:: \text { KANR; tps } 2 \Delta:: N A T^{R} ; \text { pRS415-Pab1-CFP }\end{array}$ & \\
\hline $\begin{array}{l}\text { Cdc19 }{ }^{\text {Irrev-GFP }+t p s 1 \Delta+p f k 2 \Delta} \\
+ \text { Pab1-CFP }\end{array}$ & $\begin{array}{l}\text { BY4741, CDC19::CDC19-T372D, T376D, S377D, S385D-GFP-HIS3; } \\
\text { tps1 } 1 .: N A T^{R} ; \text { pfk2 } 2:: K A N^{R} ; p R S 415-P a b 1-C F P\end{array}$ & udy \\
\hline 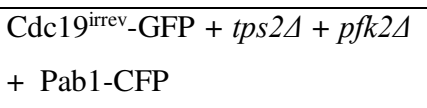 & $\begin{array}{l}\text { BY4741, CDC19::CDC19-T372D, T376D, S377D, } \\
\text { tps2 S385D-GFP-HIS3; }\end{array}$ & tudy \\
\hline $\begin{array}{l}\text { Cdc19 }{ }^{\text {Irrev }}-\text { GFP }+ \text { Pyk2OE }+ \\
\text { Pab1-CFP }\end{array}$ & $\begin{array}{l}\text { BY4741, CDC19::CDC19-T372D, T376D, S377D, S385D-GFP-HIS3; pRS416- } \\
\text { pTEF1-PYK2; pRS415-Pab1-CFP }\end{array}$ & Thi \\
\hline $\begin{array}{l}\text { Cdc19 }{ }^{\text {Irrev-GFP }} \text { Original } \\
\text { suppressor }+ \text { Pab1-CFP }\end{array}$ & $\begin{array}{l}\text { BY4741, CDC19::CDC19-T372D, T376D, S377D, S385D-GFP-HIS3; tps3- } \\
\text { G386D; pRS415-Pab1-CFP }\end{array}$ & This study \\
\hline Cdc19 ${ }^{\triangle \mathrm{FBP}}-\mathrm{GFP}+\mathrm{Pab} 1-\mathrm{CFP}$ & $\begin{array}{l}\text { BY4741, CDC19::CDC19-T402E-GFP-HIS3 ; pRS416-pTEF1-PYK2; pRS415- } \\
\text { Pab1-CFP }\end{array}$ & $\mathrm{t}$ al. \\
\hline $\begin{array}{l}\mathrm{Cdc} 9^{\triangle \mathrm{FBP}}-\mathrm{GFP}+{ }_{\text {tps } 1 \Delta+}+ \\
\text { Pab1-CFP }\end{array}$ & $\begin{array}{l}\text { BY4741, CDC19::CDC19-T402E-GFP-HIS3 ; pRS416-pTEF1-PYK2; } \\
\text { tps1 } 1:: K A N^{R} ; \text { pRS415-Pab1-CFP }\end{array}$ & This study \\
\hline
\end{tabular}


130 Suppl. Table S2: Plasmids used in this study

\begin{tabular}{|c|c|c|}
\hline Plasmid name & Genotype & Source \\
\hline Cdc19WT & pRS413-pTEF1-CDC19 & Grüning et al. \\
\hline Pyk2OE & $p R S 416-p T E F 1-P Y K 2$ & Saad et al. \\
\hline Cdc19 $19^{\mathrm{WT}}$-GFP & pRS413-pTEF1-CDC19-GFP & Saad et al. \\
\hline $\mathrm{Cdc} 19^{\triangle \mathrm{FBP}}$-GFP & pRS413-pTEF1-CDC19-T402E-GFP & Saad et al. \\
\hline Cdc19 ${ }^{\text {Not-agg_GFP }}$ & pRS413-pTEF1-CDC19-R49A-GFP & Saad et al. \\
\hline Cdc19 ${ }^{\text {irrev_GFP }}$ & pRS413-pTEF1-CDC19-T372D, T376D, S377D, S385D-GFP & Saad et al. \\
\hline GFP & pRS416-pGPD-GFP & Saad et al. \\
\hline Pab1-CFP & $p R S 415-p P A B 1-P A B 1-C F P$ & Saad et al. \\
\hline Cdc19 ${ }^{\mathrm{WT}}$-strepx2 & pET17b-CDC19-Strepx2 & Saad et al. \\
\hline Cdc19 ${ }^{\text {Not-agg }}$-strepx2 & pET17b-CDC19-R49A-Strepx2 & Saad et al. \\
\hline Cdc19 $9^{\text {irrev }}$-strepx2 & pET17b-CDC19-T372D, T376D, S377D, S385D-Strepx2 & This Study \\
\hline $\mathrm{Cdc} 19^{\triangle \mathrm{FBP}}$-strepx2 & pET17b-CDC19-T402E-Strepx2 & This study \\
\hline
\end{tabular}

131 
Figures

A
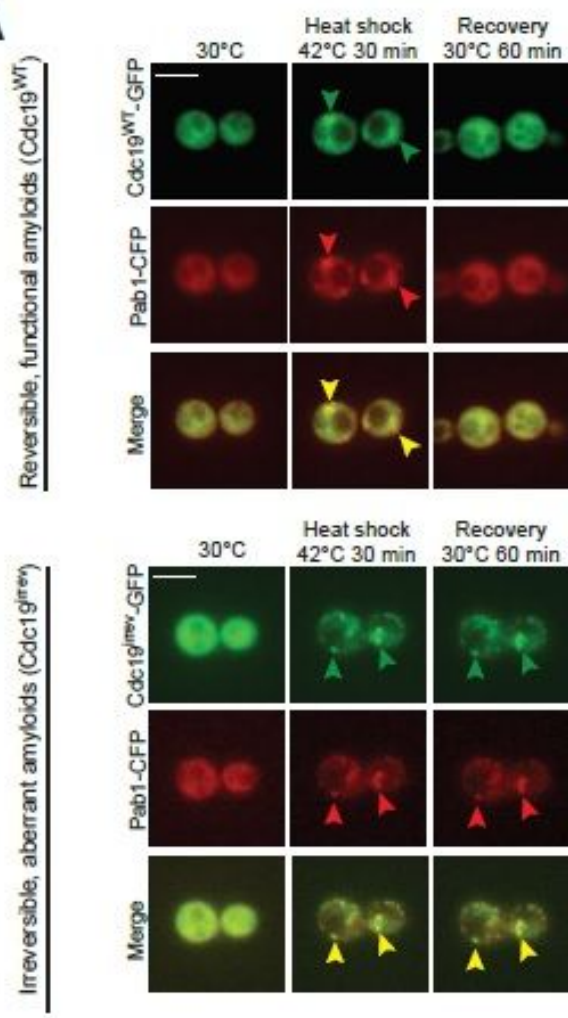

D

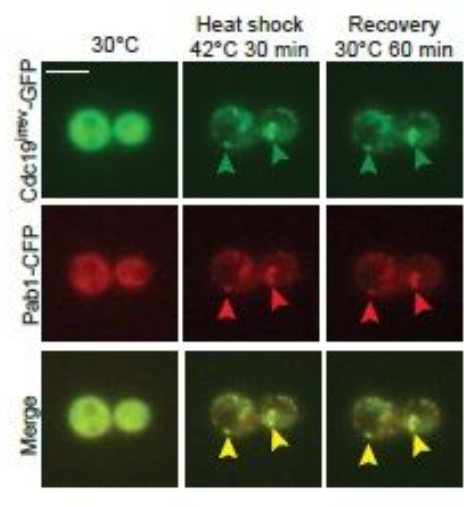

E

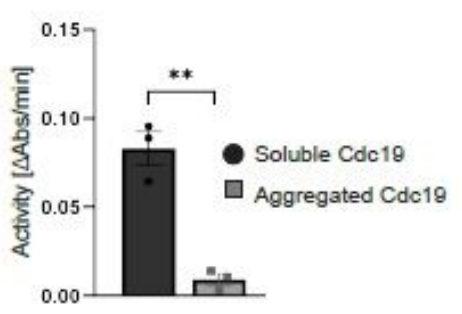

B

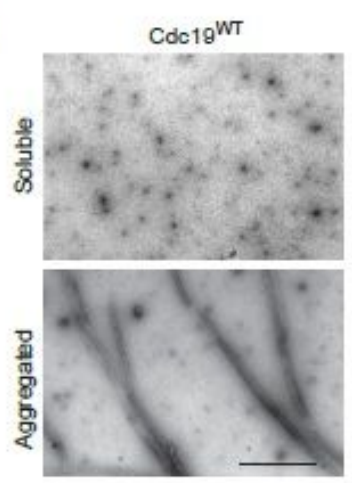

Cdc19irrev

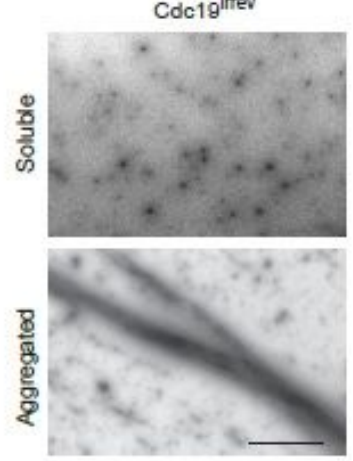

$60 \mathrm{~min}$ after stress release

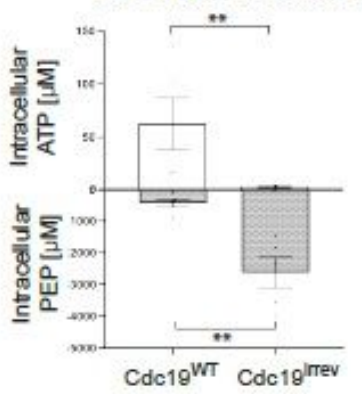

H

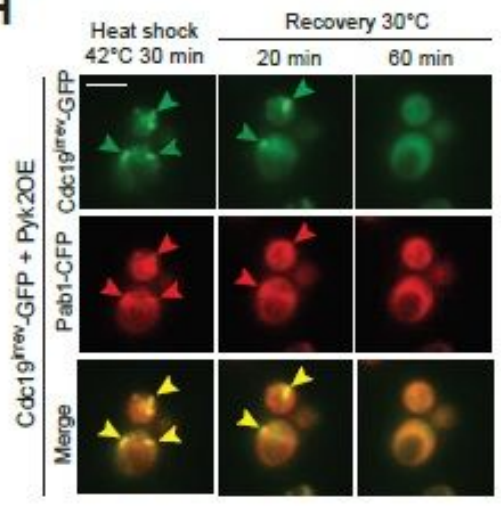

C
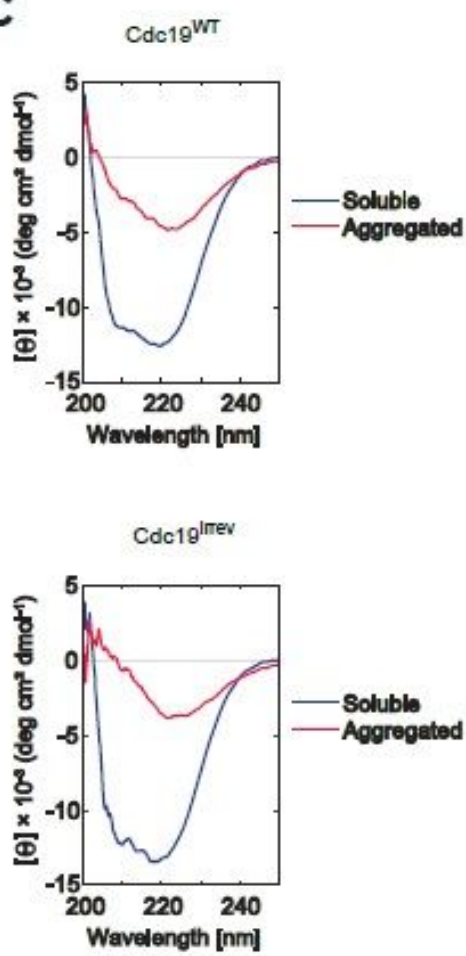

$\mathbf{F}$

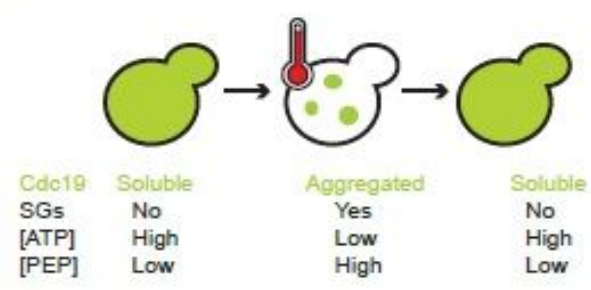

I

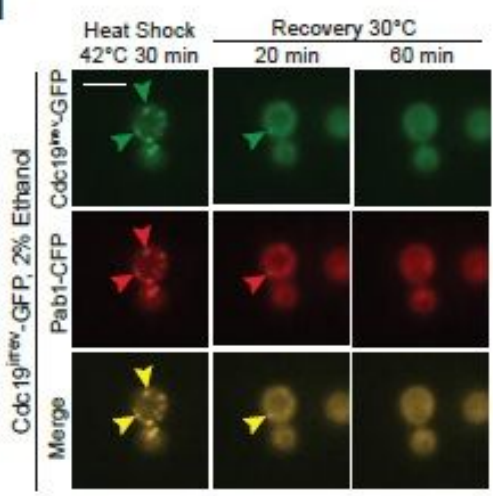

\section{Figure 1}

G disassembly depends on re-solubilization of Cdc19 amyloids and restoration of ATP production (see Manuscript File for full figure legend) 


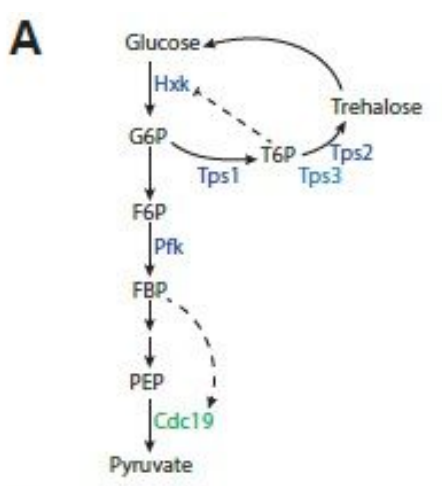

C

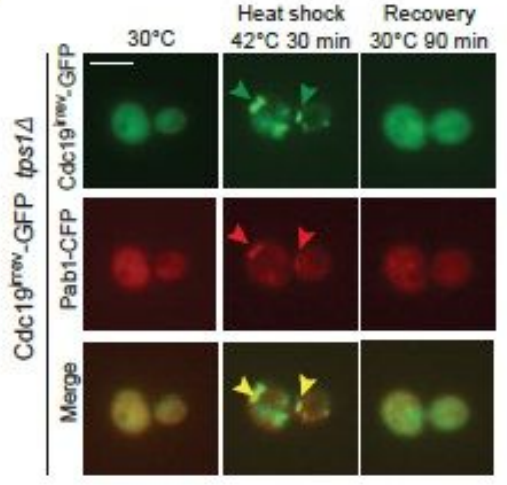

E

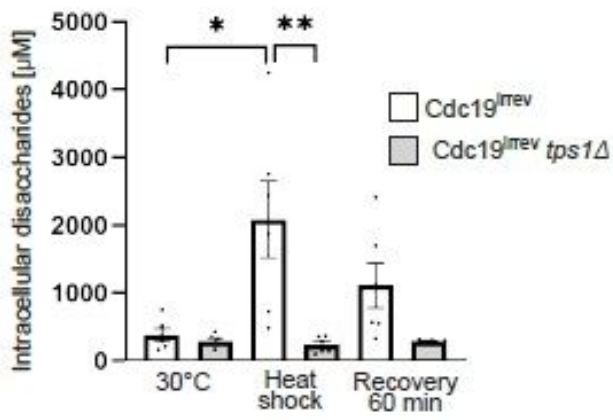

G

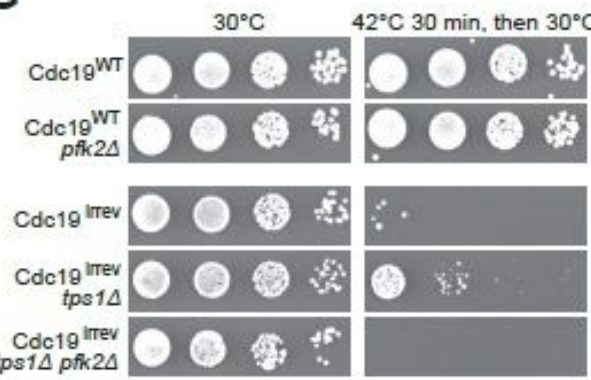

B

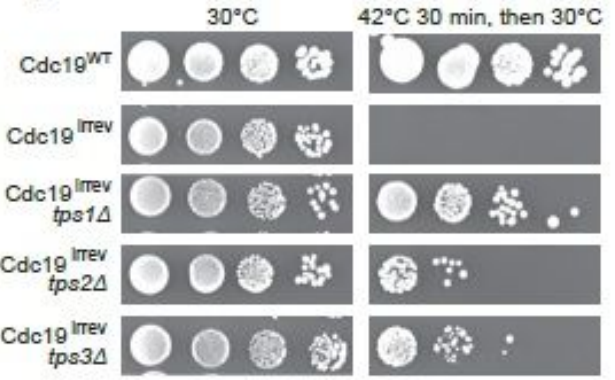

D

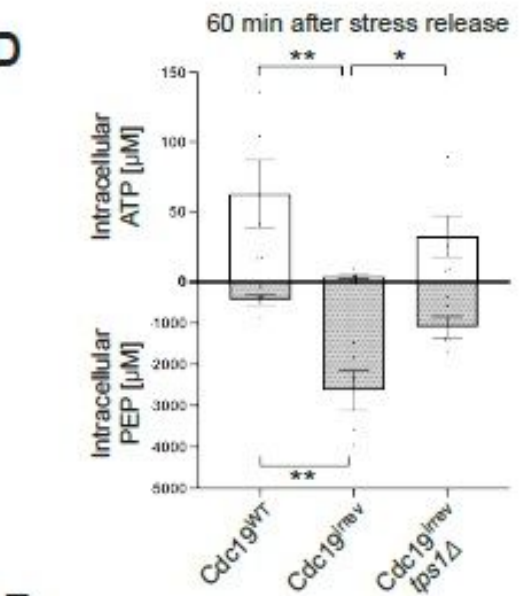

$\mathbf{F}$
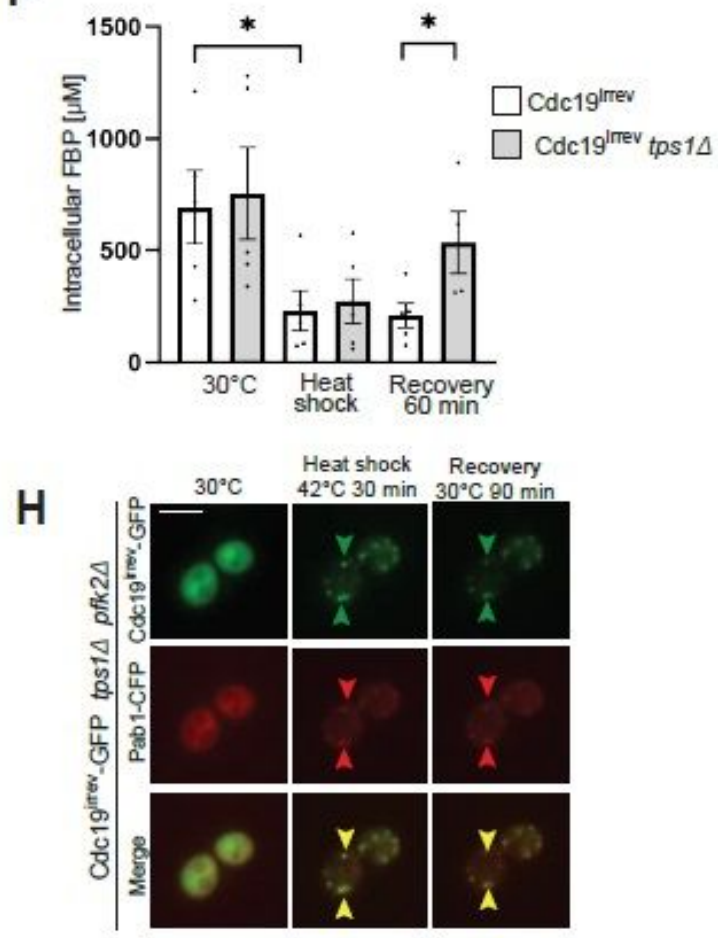

\section{Figure 2}

Re-solubilization of Cdc19irrev amyloids and cell cycle restart 901 after stress are regulated by the glycolytic metabolite FBP (see Manuscript File for full figure legend) 
A

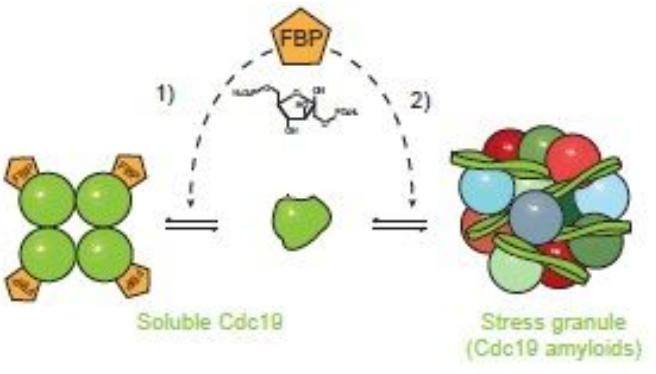

B
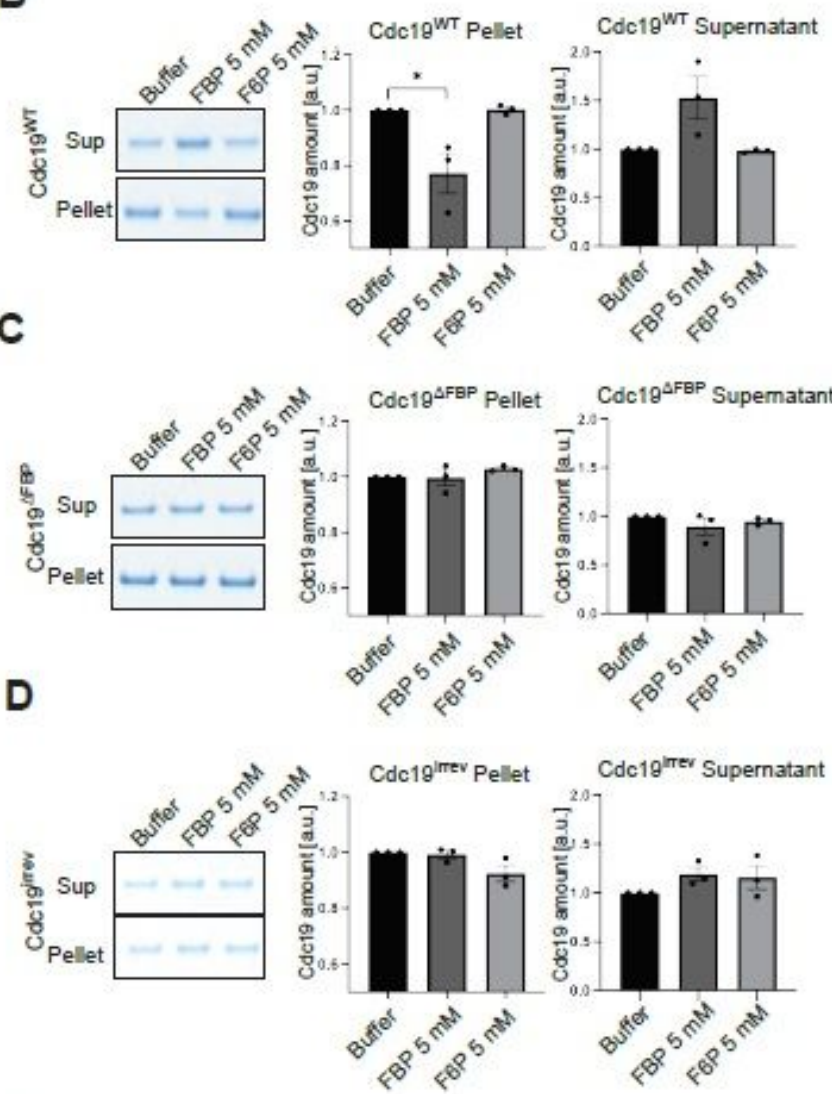

H
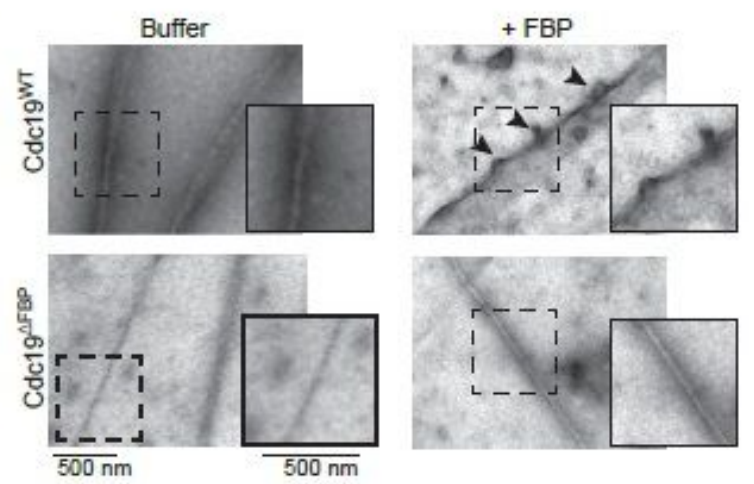

E

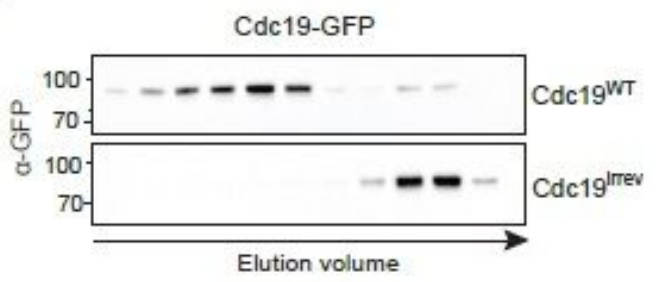

F SEC with purified Cdc19*

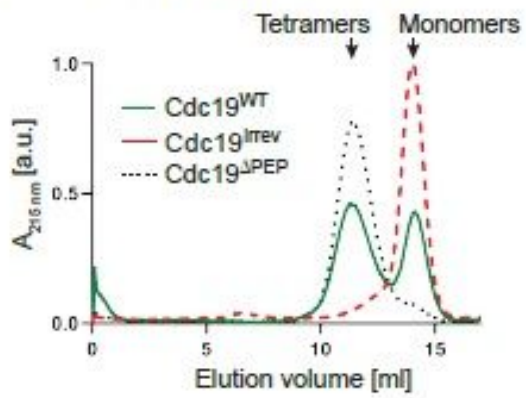

G

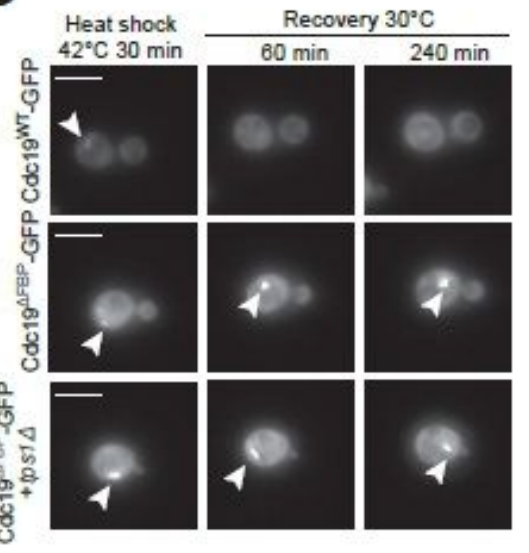

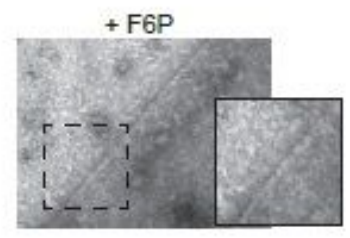

\section{Figure 3}

FBP directly regulates formation and disassembly of reversible Cdc19 amyloids(see Manuscript File for full figure legend) 
A

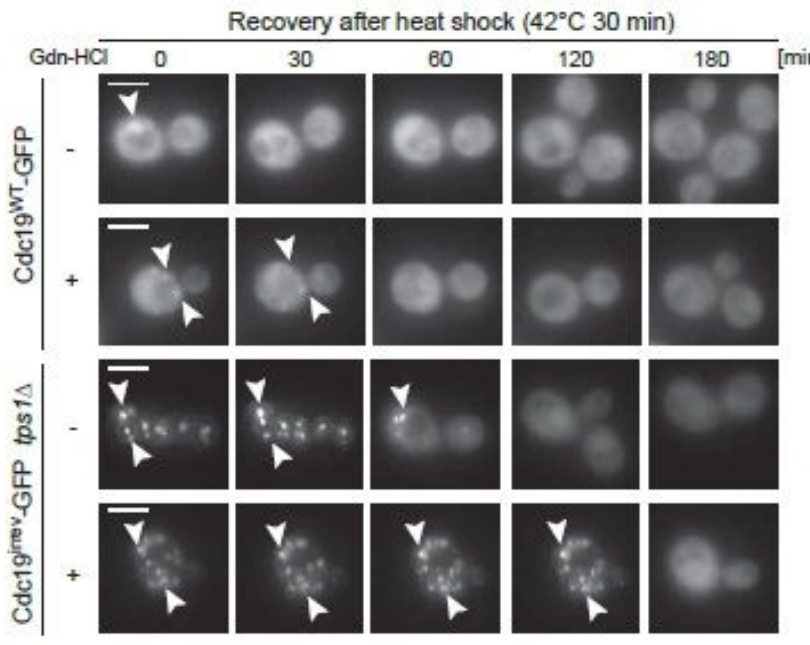

C

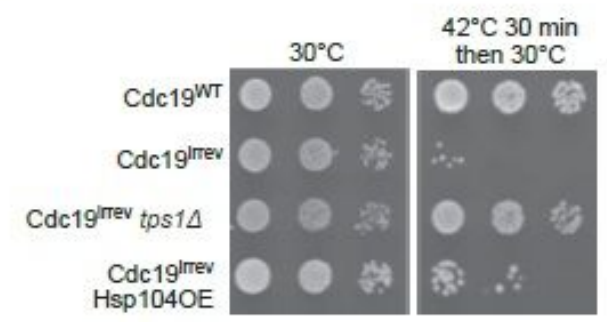

E
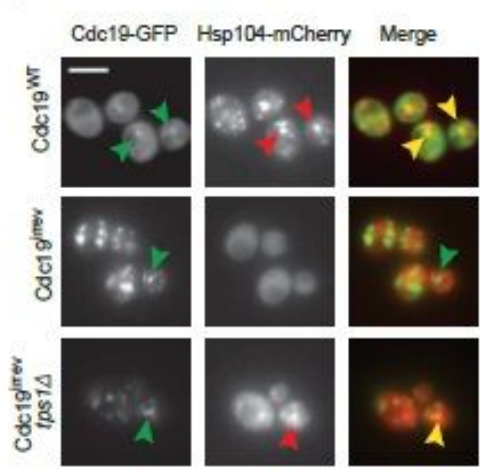

Cdc19*-GFP and Hsp104-mCherry

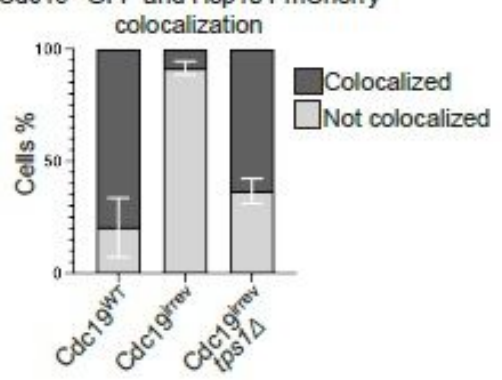

$\mathbf{F}$

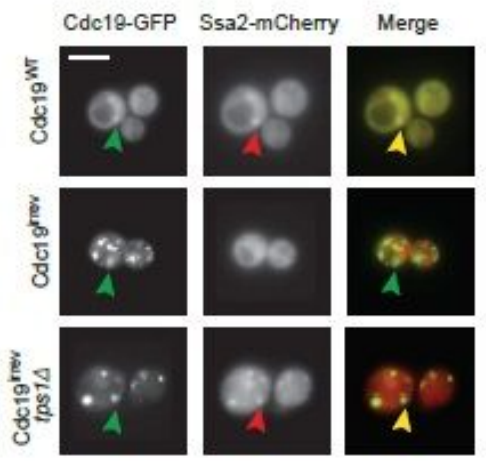

Cdc19*-GFP and Ssa2-mCherry

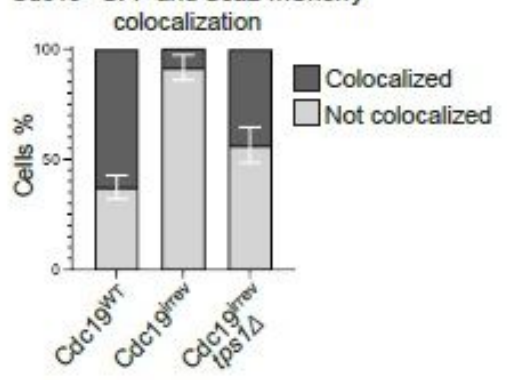

G
B
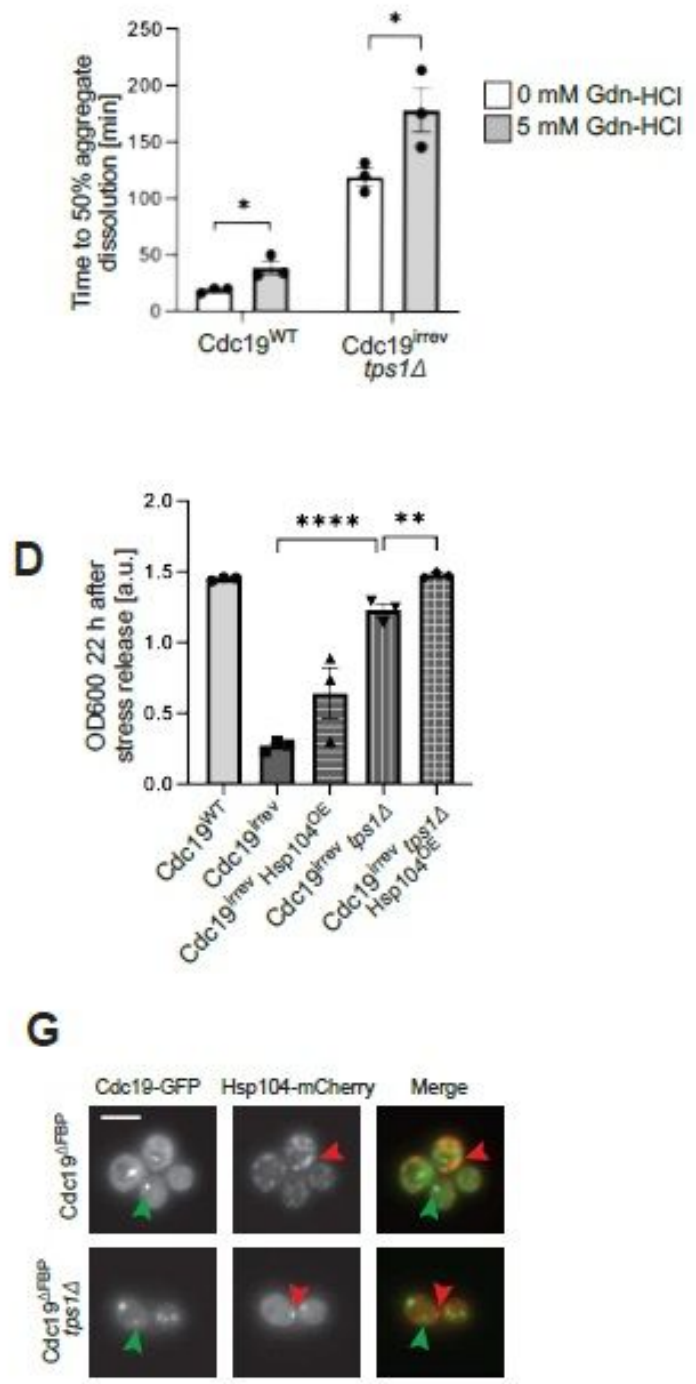

Cdc19*-GFP and Hsp104-mCherry

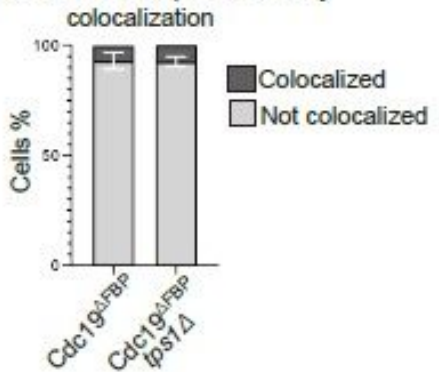

\section{Figure 4}

FBP binding to Cdc19 amyloids promotes chaperone recruitment triggering Cdc19 resolubilization and SG disassembly in vivo(see Manuscript File for full figure legend) 


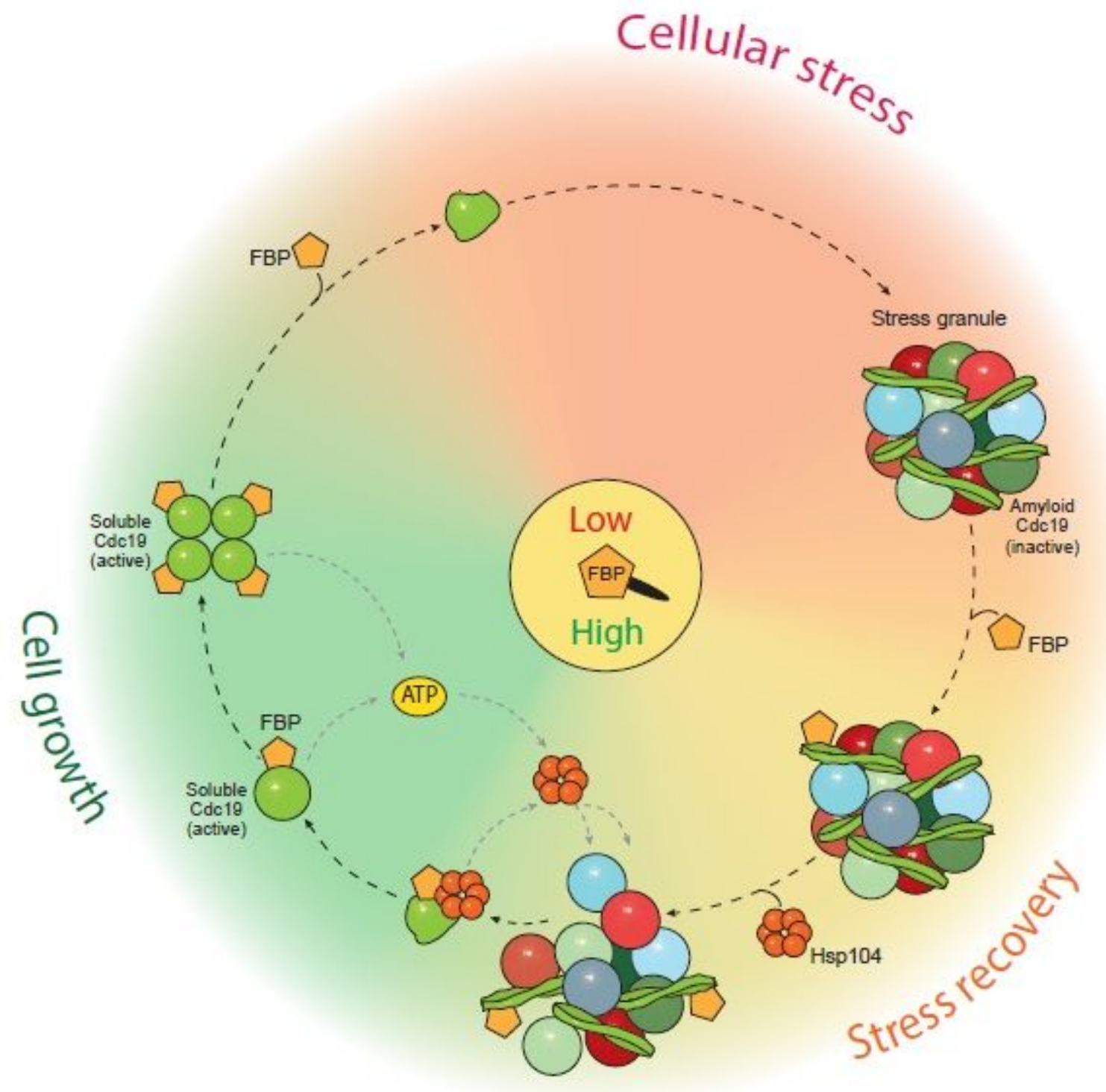

\section{Figure 5}

Molecular network coupling cellular metabolism with reversible Cdc19 amyloids and stress granule dynamics(see Manuscript File for full figure legend)

\section{Supplementary Files}

This is a list of supplementary files associated with this preprint. Click to download.

- SupplementaryTable1Strains.xlsx

- SupplementaryTable2Plasmids.xlsx 
- SupplementaryFigure1Cereghettietal.ai

- SupplementaryFigure2Cereghettietal.ai

- SupplementaryFigure3Cereghettietal.ai

- SupplementaryFigure4Cereghettietal.ai 